NBER WORKING PAPER SERIES

\title{
EQUILIBRIUM BIAS OF TECHNOLOGY
}

Daron Acemoglu

Working Paper 11845

http://www.nber.org/papers/w11845

\author{
NATIONAL BUREAU OF ECONOMIC RESEARCH \\ 1050 Massachusetts Avenue \\ Cambridge, MA 02138 \\ December 2005
}

I am grateful to Rabah Amir for several useful comments, suggestions and conversations on lattice programming, to Alexandre Debs and Asuman Ozdaglar for numerous comments and suggestions, to Alp Simsek for help with Lemma 4, and to Susan Athey, Ivan Werning, Muhamet Yildiz and seminar participants in Canadian Institute of Advanced Research, MIT, and Toulouse for useful comments. I also thank the Toulouse Network on Information Technology for financial support. The views expressed herein are those of the author(s) and do not necessarily reflect the views of the National Bureau of Economic Research.

(C2005 by Daron Acemoglu. All rights reserved. Short sections of text, not to exceed two paragraphs, may be quoted without explicit permission provided that full credit, including () notice, is given to the source. 
Equilibrium Bias of Technology

Daron Acemoglu

NBER Working Paper No. 11845

December 2005

JEL No. O30, O31, O33, C65

\begin{abstract}
$\underline{\text { ABSTRACT }}$
The study of the bias of new technologies is important both as part of the analysis of the nature of technology adoption and the direction of technological change, and to understand the distributional implications of new technologies. In this paper, I analyze the equilibrium bias of technology. I distinguish between the relative bias of technology, which concerns how the marginal product of a factor changes relative to that of another following the introduction of new technology, and the absolute bias, which looks only at the effect of new technology on the marginal product of a factor. The first part of the paper generalizes a number of existing results in the literature regarding the relative bias of technology. In particular, I show that when the menu of technological possibilities only allows for factor-augmenting technologies, the increase in the supply of a factor always induces technological change (or technology adoption) relatively biased towards that factor. This force can be strong enough to make the relative marginal product of a factor increasing in response to an increase in its supply, thus leading to an upward-sloping relative demand curve. However, I also show that the results about relative bias do not generalize when more general menus of technological possibilities are considered. In the second part of the paper, I show that there are much more general results about absolute bias. I prove that under fairly mild assumptions, an increase in the supply of a factor always induces changes in technology that are absolutely biased towards that factor, and these results hold both for small changes and large changes in supplies. Most importantly, I also determine the conditions under which the induced-technology response will be strong enough so that the price (marginal product) of a factor increases in response to an increase in its supply. These conditions correspond to a form of failure of joint concavity of the aggregate production function of the economy in factors and technology. This type of failure of joint concavity is quite possible in economies where equilibrium factor demands and technologies are decided by different agents.

Daron Acemoglu

Department of Economics

MIT, E52-380B

50 Memorial Drive

Cambridge, MA 02142-1347

and NBER

daron@mit.edu
\end{abstract}




\section{Introduction}

Despite the generally-agreed importance of technological progress for economic growth and a large and influential literature on technological progress, ${ }^{1}$ the determinants of the direction and bias of technological change are not well understood. An analysis of the direction and bias of technical change is important for a number of reasons. First, in most situations, technical change is not neutral: it benefits some factors of production, while directly or indirectly reducing the compensation of others. This possibility is illustrated both by the distributional impact of the major technologies introduced during the Industrial Revolution and the effects of technological change on the structure of wages during the past half century or so. ${ }^{2}$ The bias of technological change determines its distributional implications (i.e., which groups are the winners and which will be the losers from technological progress) and thus the willingness of different groups to embrace new technologies. Second, an understanding of the determinants of innovation requires an analysis of the bias and direction of new technologies, for example, for evaluating whether lines of previous innovations or technologies will be exploited in the future and the potential compatibility between old and new technologies. ${ }^{3}$ Finally, the bias of technology is important for understanding the macroeconomic implications of technological progress.

These and related questions have spurred a relatively large literature investigating various dimensions of the bias of technology. The pioneering study was Hicks' seminal book, The Theory of Wages (1932), which first discussed the issue of induced innovation. ${ }^{4}$ The topic later attracted attention from the leading economists of the 1960s, notably Kennedy (1964), Samuelson (1965), Drandakis and Phelps (1965), Ahmad (1966), Nordhaus (1973), David (1975), and Binswanger and Ruttan (1978), who studied the link between factor prices and technical change. The focus of this literature was on the macroeconomic consequences of induced innovation and was shaped by a critical passage in Hicks's book where he argued:

\footnotetext{
${ }^{1}$ See, among others, Dasgupta and Stiglitz (1980), Reinganum (1981, 1985), Spence (1984), and Grossman and Shapiro (1987) in the industrial organization literature and Romer (1990), Segerstrom, Anant and Dinopoulos (1990), Grossman and Helpman (1991), Aghion and Howitt (1992), Stokey (1991, 1995), and Young (1993) in the economic growth literature.

${ }^{2}$ On the biases and distributional effects of the technologies introduced during the Industrial Revolution, see Mantoux (1961) or Mokyr (1990), and on recent developments, see footnote 6 below.

${ }^{3}$ See, for example, Farrell and Saloner (1985) and Katz and Shapiro (1985).

${ }^{4}$ There is an implicit reference to this issue in Marx, when he discusses how labor scarcity-the exhaustion of the reserve army of labor - may induce the capitalist to substitute machinery for labor (see Rosenberg, 1982), and also in Habakkuk's (1962) well-known contrast of faster technological progress in the United States than in Britain because of labor scarcity in the former country (see, in particular, p. 44).
} 
"A change in the relative prices of the factors of production is itself a spur to invention, and to invention of a particular kind - directed to economizing the use of a factor which has become relatively expensive." (pp. 124-5)

Although not explicitly stated, the implicit message in this sentence (and the way it was interpreted) was that factor prices were the crucial element shaping the bias and direction of technological progress (or technological adoption), and somehow as a factor becomes more abundant, thus less expensive, technical change should become less biased towards that factor. ${ }^{5}$

The topic of biased technological change received renewed interest over the past decade, as a result of a number of macro phenomena, particularly, the evidence that overall technological change over the past 60 years has been biased towards skilled workers (e.g., Autor, Katz and Krueger, 1998). This led a number of authors to formulate extensions of endogenous growth models (Acemoglu, 1998, 2002, 2003a,b, Acemoglu and Zilibotti, 2000, Kiley, 1999, Caselli and Coleman, 2004, Xu, 2001, Gancia, 2003, Thoenig and Verdier, 2003, Ragot, 2003, Duranton, 2004, Benabou, 2005, and Jones, 2005), whereby technical change could be directed to one of multiple (typically two) sectors or factors. ${ }^{6}$

These models were descendents of the endogenous growth models of Romer, Grossman and Helpman, and Aghion and Howitt. As a result, they incorporated a number of specific features. These included a quasi-linear structure to obtain long-run growth, the constant elasticity of demand borrowed from the Dixit-Stiglitz-Spence model, different types of technologies that were of factor-augmenting type, and the market size effect, inherent in Romer's original article and present in the second generation of endogenous technical change models (see, e.g., Aghion and Howitt, 1998).

\footnotetext{
${ }^{5}$ Nevertheless, parts of Hicks's reasoning did not go uncriticized. For example, Salter (1966) and Samuelson (1965) pointed out that firms should strive to economize on total costs not only on the factor that has become relatively more expensive, thus questioning Hicks's reasoning (see also Nordhaus, 1973). But the essential ideas encapsulated in the quote remained influential in the literature. I will clarify below that this quote is never correct for factor bias, but would be true for factor-augmenting changes as long as the relevant (local) elasticity of substitution is less than one.

Another implication of this quote relates to the effect of the price of a factor, say labor, on the overall amount of technology adoption or innovation, which is also an interesting area for study, but not part of the focus of this paper. See Acemoglu and Finkelstein (2005) for a theoretical and empirical investigation of this point.

${ }^{6}$ The focus of the first papers in this literature, Acemoglu (1998) and Kiley (1999), was to investigate when and why technology could be biased towards skilled workers. This was partly motivated by the evidence that in the 19th and early 20th centuries, new technologies were often replacing skilled workers, which contrasted to the later skill-biased nature of technological change (see James and Skinner, 1985, Goldin and Katz, 1995, on the earlier era, and Goldin and Katz, 1998 or Autor, Katz and Krueger, 1998, on the more recent trends). Later Acemoglu (2003b) and Jones (2005) used similar ideas to investigate whether there are any compelling reasons for technical change to be purely labor augmenting as required for the existence of a balanced growth path in standard growth models. Acemoglu (2003a), Xu (2001), Gancia (2003), and Thoenig and Verdier (2003) used versions of this framework to investigate the effect of international trade on the bias of technology.
} 
This structure led to some very sharp results about the relative equilibrium bias of technology, which, in many ways, stood in contrast to the implicit message of Hicks's quote mentioned above. Using an endogenous growth model with two sectors and a constant elasticity of substitution between factors, Acemoglu (2002) showed that these models implied essentially the opposite conclusion to that of Hicks's quote. To describe these results, let relative bias, in a two-factor world, be the impact of new technology on the marginal product of a factor relative to that of the other. ${ }^{7}$ The main result in this class of models is that when a factor becomes more abundant, technology becomes endogenously more (relatively) biased towards that factor.

The question that arises naturally is whether these results are an artifact of special assumptions imposed in this class of models. Understanding the source of existing results is not only important for deriving general theorems about equilibrium bias, but also because without such an understanding, the forces determining the nature of technology adoption and technological progress remain unclear. The purpose of this paper is to provide an in-depth analysis of equilibrium bias and provide general theorems in the most natural setting that allows an analysis of these questions.

To motivate the analysis, we may return to the results from Acemoglu (2002) mentioned above, and wonder whether those results hold in more general settings. In particular, we may start with the following conjecture:

Conjecture (Relative Endogenous Bias): When the supply of a factor $Z$ increases, technology becomes relatively more biased towards factor $Z$.

The first theorem in this paper shows that this conjecture is correct in a world with two factors and two factor-augmenting technologies, thus generalizing existing results. Moreover, it will provide precise conditions for this relative bias to be strong, i.e., for the increase in the relative supply of a factor to increase its relative price once technology has adjusted to the change in factor supplies. The second result, however, is that once we depart from an environment in which all technologies are of the factor-augmenting kind, this conjecture is no longer true. It is possible to construct relatively simple examples where it fails.

Despite this negative result, the main results in the paper, presented in the second part, are a series of positive and fairly general results about equilibrium bias. In contrast to the focus in Acemoglu $(1998,2002)$ and the conjecture above, these results concern the "absolute", not relative, bias. A technology is said to be absolutely biased towards a factor if it increases its

\footnotetext{
${ }^{7}$ Equivalently, bias can be described as referring to cost-minimizing relative factor demands at a given factor price ratio. The two definitions of relative bias are equivalent for the purposes in this paper.
} 
marginal product. ${ }^{8}$ While understanding relative bias is essential for a certain class of questions (for example, those concerning inequality), an analysis of absolute bias is equally important, for example, for understanding the implications of technological change for the level of wages or the level of rewards to other factors.

One of the main results of the paper is the following theorem which is stated loosely and without the necessary assumptions here (which are explained subsequently):

Theorem (Weak Endogenous Bias): When the supply of a factor $Z$ increases, technology becomes absolutely biased towards factor $Z$.

Stated differently, this theorem shows that under a set of relatively mild assumptions on the underlying environment, there is a strong result about equilibrium bias; technology will progressively favor factors that are becoming abundant. I will show that this theorem applies under two alternative sets of sufficient conditions. The first set of conditions requires the measure (vector) of technology to belong to a convex subset of $\mathbb{R}^{K}$ for some $K \geq 1$, which will lead to a local theorem (i.e., a result that applies in response to small changes in the supply of a factor $Z$ ). The second possibility is a global theorem. The conditions necessary for this version of the theorem can be best understood by using the tools of monotone comparative statics as developed by Topkis (1978, 1979, 1998), Milgrom and Roberts (1990a,b, 1994), Vives (1990), and Milgrom and Shannon (1994). In fact, the sufficient condition for the global theorem is a form of supermodularity (or increasing differences) between factor $Z$ (or a set of factors, $\left.Z_{1}, \ldots, Z_{N}\right)$ and a vector $\theta$ denoting technology choices.

These results are not only interesting because of their generality, but also because they shed light on a variety of real-world phenomena. For example, they suggest why recent technical change may have increased the demand for skilled workers (since there has been a significant increase in the number of more educated workers), and why technological progress may have been biased towards unskilled workers in the past (since there was a large increase in the supply of unskilled workers in British cities during the 19th century, see, for example, Habakkuk 1962, or Williamson, 1990).

The above theorem is referred to as the "weak endogenous bias" theorem, because it only specifies the direction of the bias. Perhaps more important and certainly more surprising is the following theorem:

Theorem (Strong Endogenous Bias): When the aggregate production possibilities set of the economy is nonconvex, an increase in the supply of a factor $Z$ induces technology to

\footnotetext{
${ }^{8}$ Thus the difference is that this marginal product is not compared to the marginal product of other factors.
} 
become sufficiently biased towards factor $Z$ so as to increase its equilibrium price.

In other words, when the production possibilities set is nonconvex, equilibrium bias will be strong enough that endogenous-technology demand curves for factors will be upward-sloping rather than downward-sloping as in the standard neoclassical theory. This result will be stated in Theorem 8 below. Puts differently, there will be strong (absolute) equilibrium bias if and only if the aggregate output of the economy (or a transformation of it) fails to be jointly concave in technology (say $\theta$ ) and $Z$ (which is the essence of the nonconvexity mentioned above). In equilibrium output (profits) will be maximized in the choice of $Z$ by firms, while the choice of technology $\theta$ by some other agents (a technology monopolist or research firm) will also maximize output (or some transform thereof). Nevertheless, these two conditions together do not guarantee that the equilibrium, say $\left(Z^{*}, \theta^{*}\right)$, is a maximum of the aggregate output (or its transform). Instead, $\left(Z^{*}, \theta^{*}\right)$ could be a saddle point, meaning that there exists a direction in the $(Z, \theta)$ plane in which aggregate output increases. Essentially, Theorem 8 will show that there will be strong equilibrium bias whenever this is the case, and strong bias will never exist when $\left(Z^{*}, \theta^{*}\right)$ is in fact a maximum. This implies that the equilibrium structure, where technology and factor demands are chosen by different agents in the economy is essential for this result, since otherwise, $\left(Z^{*}, \theta^{*}\right)$ would be a maximum, thus ruling out strong bias. Equally important, however, is the observation that once we have an economy in which factor demands and technology are chosen by different agents, such strong bias is quite easy to obtain, because there is nothing that rules out nonconvexities or guarantees that $\left(Z^{*}, \theta^{*}\right)$ should be a maximum in all directions.

In addition to the earlier work on induced innovation literature and the recent directed technical change literature that have already been discussed above, this paper is closely related to work on the LeChatelier principle. Recall that the LeChatelier principle concerns the demand for factors of a profit-maximizing firm, and states that long-run demand curves (which allow adjustment in all factors) are more elastic than short-run demand curves (which hold the employment level of other factors constant). In other words, in response to an increase in the price of a factor, the employment of this factor declines more in the short-run than in the long-run. This principle was first stated and proved by Samuelson (1947) for small changes in factor prices, but was known not to be true for large changes (see, for example, Samuelson, 1960, Roberts, 1999). It was later generalized by Milgrom and Roberts (1996) to a global LeChatelier principle under the assumption that production functions are supermodular (see also Silberberg, 1974). The intuition underlying the LeChatelier principle is that the firm can 
adjust other factors to increase the marginal product of the factor whose price has increased. At some level, results about the endogenous bias of technology correspond to equilibrium versions of the LeChatelier principle. The main difference is that the focus here is the effect of changes in factor supplies on equilibrium outcomes, rather than the partial equilibrium/optimization focus of the LeChatelier principle. The above discussion illustrates that this equilibrium structure is responsible for the possibility of strong equilibrium bias (since a firm's demand curve for a factor can never be upward sloping, even in the long run, see, e.g., Mas-Colell, Whinston and Green, 1995, Proposition 5.C.2). Equivalently, as discussed above, strong equilibrium bias requires technology and factor demands to be chosen by different agents.

The rest of the paper is organized as follows. In Section 2, I describe three alternative environments, with different market structures and assumptions on technology choice, and show that the determination of equilibrium bias in these three different economies boils down to the same problem, with the major difference that two of the economies allow for more natural nonconvexities in the aggregate production possibilities set. Section 3 provides a generalization of existing relative bias results, but also shows why the conjecture regarding relative bias above is not correct unless we restrict the technology possibilities menu to only factor-augmenting technologies. The main results of the paper are contained in Section 4 and 5. Section 4 presents a number of versions of the theorems on weak equilibrium (absolute) bias and also clarifies the limits of these theorems. Section 5 contains the results on strong equilibrium bias. Section 6 concludes, while Appendices A and B contain some additional technical material.

\section{The Basic Environments}

Consider a static economy consisting of a unique final good and two sets of factors of production, a total of $N+M, \mathbf{Z}=\left(Z_{1}, \ldots, Z_{N}\right)$ and $\mathbf{L}=\left(L_{1}, \ldots, L_{M}\right)$. Throughout, I assume that all agents' preferences are defined over the consumption of the final good. Moreover, all factors are supplied inelastically and denote their supplies by $\overline{\mathbf{Z}} \in \mathbb{R}_{+}^{N}$ and $\overline{\mathbf{L}} \in \mathbb{R}_{+}^{M}$. The reason for distinguishing between these two sets of factors is to carry out comparative static exercises varying the supply of factors $\mathbf{Z}$, while holding the supply of other factors, $\mathbf{L}$, constant. The economy consists of a continuum of firms (final good producers) denoted by the set $\mathcal{F}$, each with an identical production function. Without loss of any generality let us normalize the measure of $\mathcal{F},|\mathcal{F}|$, to 1 . The price of the final good is also normalized to $1 .^{9}$

\footnotetext{
${ }^{9}$ Since all agents' preferences are defined over the final good, ownership of firms is not important for the equilibrium allocations. In particular, firms will always maximize profits independent of their exact ownership
} 
I will consider three different environments to highlight the importance of convexity of the aggregate production set. All three environments will lead to a similar structure for the determination of equilibrium bias. In particular, they will all generate the weak equilibrium bias under the set of conditions already discussed in the Introduction, but two of them can generate the strong equilibrium bias more naturally (see below for formal definitions).

The first, Economy D (for decentralized), is a completely decentralized economy in which technologies are chosen by firms themselves. In some ways, in this economy, technology choice can be interpreted as choice of just another set of factors. This economy also has some similarity to the models recently analyzed by Boldrin and Levine (2001, 2004) and Quah (2003), which emphasize the possibility of endogenous technological change without monopolistic competition. But from the point of view of this paper, the most important aspect of Economy D is that the whole discussion can be in terms of technology adoption, and we can work with a convex decentralized economy familiar from basic general equilibrium analysis.

The second, Economy C (for centralized), features a benevolent social planner choosing the technology. The third is, in many ways, the most standard environment, and features a monopolist choosing and selling technologies. This environment, Economy M (for monopoly), will lead to identical results to Economy C. A tradition dating back to Schumpeter (1934) and Arrow (1962), and more recently used by Romer (1990), Grossman and Helpman (1991), and Aghion and Howitt (1992), emphasizes both the non-rivalrous nature of new technologies and the monopoly power necessary to recoup the investments made for $R \& D$, and these features are captured in Economy $\mathrm{M}$ in a simple manner.

\subsection{Economy D-Decentralized Equilibrium}

In the first environment, Economy D, all markets are competitive and technology is decided by each firm separately. In this case, each firm $i \in \mathcal{F}$ has access to a production function

$$
Y^{i}=F\left(\mathbf{Z}^{i}, \mathbf{L}^{i}, \theta^{i}\right)
$$

where $\mathbf{Z}^{i} \in \mathcal{Z} \subset \mathbb{R}_{+}^{N}, \mathbf{L}^{i} \in \mathcal{L} \subset \mathbb{R}_{+}^{M}$ and $\theta^{i} \in \Theta$ is the measure of technology. $F$ is a real-valued production function, which, for simplicity, I take to be twice continuously differentiable in $\left(\mathbf{Z}^{i}, \mathbf{L}^{i}\right){ }^{10}$ For now I impose no structure on the set $\Theta$, but for concreteness, one might think structure. For this reason, I do not specify the ownership structure of firms in what follows.

${ }^{10}$ Whenever $F$ is assumed to be differentiable in $\left(\mathbf{Z}^{i}, \mathbf{L}^{i}\right)\left[\left(\mathbf{Z}^{i}, \mathbf{L}^{i}, \theta\right)\right]$, this means that it is differentiable over some open set containing $\mathcal{Z} \times \mathcal{L}[\mathcal{Z} \times \mathcal{L} \times \Theta]$.

The differentiability assumptions are not necessary for the main results, and only facilitate the exposition by allowing a clear definition of marginal products and factor prices. Without differentiability, factor prices (marginal products) can take values in the set of generalized Clarke derivatives as defined in Clarke (1990). 
of $\Theta \subset \mathbb{R}^{K}$ for some $K \in \mathbb{N}$. For many instances of technology choice, $\Theta$ may consist of discrete elements (corresponding to separate technologies), so it may not be a convex set. For the global results, we will need that both $\Theta$ and $\mathcal{Z}$ are lattices according to some order. ${ }^{11}$

Each final good producer (firm) maximizes profits, i.e., it solves the problem:

$$
\max _{\mathbf{Z}^{i} \in \mathcal{Z}, \mathbf{L}^{i} \in \mathcal{L}, \theta_{i} \in \Theta} \pi\left(\mathbf{Z}^{i}, \mathbf{L}^{i}, \theta^{i}\right)=F\left(\mathbf{Z}^{i}, \mathbf{L}^{i}, \theta^{i}\right)-\sum_{j=1}^{N} w_{Z j} Z_{j}^{i}-\sum_{j=1}^{M} w_{L j} L_{j}^{i},
$$

where $w_{Z j}$ is the price of factor $Z_{j}$ for $j=1, \ldots, N$, and $w_{L j}$ is the price of factor $L_{j}$ for $j=1, \ldots, M$, all taken as given by the firm. Similar to the notation for $L$ and $Z$, I will use $\mathbf{w}_{Z}$ and $\mathbf{w}_{L}$ to denote the vector of factor prices. Since there is a total supply $\bar{Z}_{j}$ of factor $Z_{j}$ and a total supply $\bar{L}_{j}$ of factor $L_{j}$, and both factors are supplied inelastically, market clearing requires

$$
\int_{i \in \mathcal{F}} Z_{j}^{i} d i \leq \bar{Z}_{j} \text { for } j=1, \ldots, N \text { and } \int_{i \in \mathcal{F}} L_{j}^{i} d i \leq \bar{L}_{j} \text { for } j=1, \ldots, M
$$

Definition 1 A competitive equilibrium in Economy D is a set of decisions $\left\{\mathbf{Z}^{i}, \mathbf{L}^{i}, \theta^{i}\right\}_{i \in \mathcal{F}}$ and factor prices $\left(\mathbf{w}_{Z}, \mathbf{w}_{L}\right)$ such that $\left\{\mathbf{Z}^{i}, \mathbf{L}^{i}, \theta^{i}\right\}_{i \in \mathcal{F}}$ solve (2) given prices $\left(\mathbf{w}_{Z}, \mathbf{w}_{L}\right)$ and (3) holds.

I refer to any $\theta^{i}$ that is part of the set of equilibrium allocations, $\left\{\mathbf{Z}^{i}, \mathbf{L}^{i}, \theta^{i}\right\}_{i \in \mathcal{F}}$, as "equilibrium technology".

Assumption $\mathbf{1} \Theta \subset \mathbb{R}^{K}$ for some $K \geq 1, F\left(\mathbf{Z}^{i}, \mathbf{L}^{i}, \theta^{i}\right)$ is jointly strictly concave in $\left(\mathbf{Z}^{i}, \mathbf{L}^{i}, \theta^{i}\right)$ and increasing in $\left(\mathbf{Z}^{i}, \mathbf{L}^{i}\right)$, and $\mathcal{Z}, \mathcal{L}$ and $\Theta$ are convex.

Then by standard arguments we have:

Lemma 1 (Symmetry) Suppose Assumption 1 holds. Then in any competitive equilibrium, $\left(\mathbf{Z}^{i}, \mathbf{L}^{i}, \theta^{i}\right)=(\overline{\mathbf{Z}}, \overline{\mathbf{L}}, \theta)$ for all $i \in \mathcal{F}$.

Proof. This lemma follows immediately by the strict concavity of $F\left(\mathbf{Z}^{i}, \mathbf{L}^{i}, \theta^{i}\right)$, which implies strict concavity of $\pi\left(\mathbf{Z}^{i}, \mathbf{L}^{i}, \theta^{i}\right)$. To obtain a contradiction, suppose that two firms, $i$ and $i^{\prime}$, choose $\left(\mathbf{Z}^{i}, \mathbf{L}^{i}, \theta^{i}\right)$ and $\left(\mathbf{Z}^{i^{\prime}}, \mathbf{L}^{i^{\prime}}, \theta^{i^{\prime}}\right)$, such that $\left(\mathbf{Z}^{i}, \mathbf{L}^{i}, \theta^{i}\right) \neq\left(\mathbf{Z}^{i^{\prime}}, \mathbf{L}^{i^{\prime}}, \theta^{i^{\prime}}\right)$. This is only

\footnotetext{
${ }^{11}$ Since $\mathcal{Z}$ is a subset of $\mathbb{R}_{+}^{N}$, an easy way to guarantee that it is a lattice is to assume it to be a "boxconstrained" region (or a cube) with a minimum and maximum value for each $Z_{j}^{i}$. Although the lattice structure can be restrictive under some circumstances, for example when there are budget-type relationships between subcomponents of the vector, it is not very restrictive in this context, since the fact that a firm is hiring more of one factor does not typically put constraints on its hiring more of others.
} 
possible if $\pi\left(\mathbf{Z}^{i}, \mathbf{L}^{i}, \theta^{i}\right)=\pi\left(\mathbf{Z}^{i^{\prime}}, \mathbf{L}^{i^{\prime}}, \theta^{i^{\prime}}\right)$. Now consider the vector $(\mathbf{Z}, \mathbf{L}, \theta)=\lambda\left(\mathbf{Z}^{i}, \mathbf{L}^{i}, \theta^{i}\right)+$ $(1-\lambda)\left(\mathbf{Z}^{i^{\prime}}, \mathbf{L}^{i^{\prime}}, \theta^{i^{\prime}}\right)$ for some $\lambda \in(0,1)$, which is feasible by the convexity of $\mathcal{L}, \mathcal{Z}$ and $\Theta$. Strict concavity implies that $\pi(\mathbf{Z}, \mathbf{L}, \theta)>\lambda \pi\left(\mathbf{Z}^{i}, \mathbf{L}^{i}, \theta^{i}\right)+(1-\lambda) \pi\left(\mathbf{Z}^{i^{\prime}}, \mathbf{L}^{i^{\prime}}, \theta^{i^{\prime}}\right)$, hence $\pi(\mathbf{Z}, \mathbf{L}, \theta)>$ $\pi\left(\mathbf{Z}^{i}, \mathbf{L}^{i}, \theta^{i}\right)=\pi\left(\mathbf{Z}^{i^{\prime}}, \mathbf{L}^{i^{\prime}}, \theta^{i^{\prime}}\right)$, delivering a contradiction. Therefore for all $i \in \mathcal{F}$, we have $\left(\mathbf{Z}^{i}, \mathbf{L}^{i}, \theta^{i}\right)=(\mathbf{Z}, \mathbf{L}, \theta)$. Since $F$ is increasing in $\left(\mathbf{Z}^{i}, \mathbf{L}^{i}\right)$, market clearing, (3), and $|\mathcal{F}|=1$ imply that $(\mathbf{Z}, \mathbf{L})=(\overline{\mathbf{Z}}, \overline{\mathbf{L}})$, completing the proof.

Assumption 1 may be restrictive, however, because it rules out constant returns to scale in $\left(\mathbf{Z}^{i}, \mathbf{L}^{i}, \theta^{i}\right)$. Alternatively, we can modify this assumption to allow for constant returns to scale: ${ }^{12}$

Assumption 1' $\Theta \subset \mathbb{R}^{K}$ for some $K \geq 1, F\left(\mathbf{Z}^{i}, \mathbf{L}^{i}, \theta^{i}\right)$ is increasing in $\left(\mathbf{Z}^{i}, \mathbf{L}^{i}\right)$ and exhibits constant returns to scale in $\left(\mathbf{Z}^{i}, \mathbf{L}^{i}, \theta^{i}\right)$, and we have $(\overline{\mathbf{Z}}, \overline{\mathbf{L}}) \in \mathcal{Z} \times \mathcal{L}$.

Proposition 1 (Welfare Theorem D) Suppose Assumption 1 or Assumption 1' holds. Then any equilibrium technology $\theta$ is a solution to

$$
\max _{\theta^{\prime} \in \Theta} F\left(\overline{\mathbf{Z}}, \overline{\mathbf{L}}, \theta^{\prime}\right)
$$

and any solution to this problem is an equilibrium technology.

Proof. ( $\Longrightarrow$ First suppose Assumption 1 holds. Suppose that $\left\{\mathbf{Z}^{i}, \mathbf{L}^{i}, \theta^{i}\right\}_{i \in \mathcal{F}}$ is a competitive equilibrium. By Lemma $1,\left\{\mathbf{Z}^{i}, \mathbf{L}^{i}, \theta^{i}\right\}_{i \in \mathcal{F}}$ is such that $\left(\mathbf{Z}^{i}, \mathbf{L}^{i}, \theta^{i}\right)=(\overline{\mathbf{Z}}, \overline{\mathbf{L}}, \boldsymbol{\theta})$ for all $i \in \mathcal{F}$. Moreover, by the definition of a competitive equilibrium, there exist $\mathbf{w}_{Z}$ and $\mathbf{w}_{L}$ such that

$$
(\overline{\mathbf{Z}}, \overline{\mathbf{L}}, \theta) \in \arg \max _{\mathbf{Z}^{i} \in \mathcal{Z}, \mathbf{L}^{i} \in \mathcal{L}, \theta_{i} \in \Theta} F\left(\mathbf{Z}^{i}, \mathbf{L}^{i}, \theta^{i}\right)-\sum_{j=1}^{N} w_{Z j} Z_{j}^{i}-\sum_{j=1}^{M} w_{L j} L_{j}^{i} .
$$

This implies that any equilibrium technology $\theta$ satisfies $\theta \in \arg \max _{\theta^{\prime} \in \Theta} F\left(\overline{\mathbf{Z}}, \overline{\mathbf{L}}, \theta^{\prime}\right)$. Next, suppose that Assumption 1' holds. In that case, without loss of any generality, we can consider an equilibrium with only one (representative) firm active and employing $(\overline{\mathbf{Z}}, \overline{\mathbf{L}}) \in \mathcal{Z} \times \mathcal{L}$. Consequently, by the definition of a competitive equilibrium (5) holds. Thus the same conclusion follows.

$(\Longleftarrow)$ First suppose that Assumption 1 holds. Take $\theta \in \arg \max _{\theta^{\prime} \in \Theta} F\left(\overline{\mathbf{Z}}, \overline{\mathbf{L}}, \theta^{\prime}\right)$. By the strict concavity of $F$, the first-order conditions of (5) are necessary and sufficient. Consider the

\footnotetext{
${ }^{12}$ It is also possible to allow for mixtures of constant returns to scale and strict convexity, but this introduces additional notation, and since it is not essential for the focus here, I simplify the analysis by using either Assumption 1 or Assumption 1'.
} 
factor price vectors $\mathbf{w}_{Z}$ and $\mathbf{w}_{L}$ such that $w_{Z j}=\partial F(\overline{\mathbf{Z}}, \overline{\mathbf{L}}, \theta) / \partial Z_{j}$ and $w_{L j}=\partial F(\overline{\mathbf{Z}}, \overline{\mathbf{L}}, \theta) / \partial L_{j}$. The hypothesis (4) implies that at these factor price vectors, $\left(\mathbf{Z}^{i}, \mathbf{L}^{i}, \theta^{i}\right)=(\overline{\mathbf{Z}}, \overline{\mathbf{L}}, \theta)$ for all $i \in \mathcal{F}$ satisfies the first-order conditions of (5), so it is a competitive equilibrium, thus $\theta$ is an equilibrium technology. Next, suppose that Assumption 1' holds. Once again, we can consider an equilibrium with only one firm active employing $(\overline{\mathbf{Z}}, \overline{\mathbf{L}}) \in \mathcal{Z} \times \mathcal{L}$, so any $\theta \in$ $\arg \max _{\theta^{\prime} \in \Theta} F\left(\overline{\mathbf{Z}}, \overline{\mathbf{L}}, \theta^{\prime}\right)$ is an equilibrium technology, completing the proof.

Proposition 1 is useful since it enables us to focus on a simple maximization problem rather than an equilibrium problem. An important implication of this proposition is also that the equilibrium corresponds to a maximum of $F$ in the entire vector $\left(\mathbf{Z}^{i}, \mathbf{L}^{i}, \theta^{i}\right)$. It is also straightforward to see that equilibrium factor prices in this economy are equal to the marginal products of the $F$ function, and are given by $w_{Z j}=\partial F(\overline{\mathbf{Z}}, \overline{\mathbf{L}}, \theta) / \partial Z_{j}$ and $w_{L j}=$ $\partial F(\overline{\mathbf{Z}}, \overline{\mathbf{L}}, \theta) / \partial L_{j}$ where $\theta$ is the equilibrium technology choice.

We next derive a similar maximization problem for Economies $\mathrm{C}$ and $\mathrm{M}$, which relax the strong (joint) convexity assumptions inherent in Economy D, and show that a similar equilibrium characterization can be obtained for these economies, but without the implication that the equilibrium corresponds to a maximum of $F$ in the entire vector $\left(\mathbf{Z}^{i}, \mathbf{L}^{i}, \theta^{i}\right)$.

\subsection{Economy C-Centralized Equilibrium}

In this economy, there is still a unique final good and each firm has access to the production function

$$
Y^{i}=G\left(\mathbf{Z}^{i}, \mathbf{L}^{i}, \theta^{i}\right)
$$

In particular, we again have $\mathbf{Z}^{i} \in \mathcal{Z} \subset \mathbb{R}_{+}^{N}, \mathbf{L}^{i} \in \mathcal{L} \subset \mathbb{R}_{+}^{M}$ and $\theta^{i} \in \Theta$ is the measure of technology, and $G$ is again a real-valued production function that is twice continuously differentiable in $\left(\mathbf{Z}^{i}, \mathbf{L}^{i}\right)$.

Each firm has free access to the technology $\theta$ provided by the centralized (socially-run) research firm. This research firm can create any technology $\theta$ at $\operatorname{cost} C(\theta)$ from the available technology menu $\Theta$. Once created, this technology is non-excludable and available to any firm (as well as non-rival, see Arrow, 1962, Romer, 1990). In addition, to further simplify the analysis, I assume that the research firm can only choose one technology, which might be, for example, because of the necessity of standardization across firms. ${ }^{13}$

\footnotetext{
${ }^{13}$ In general, a social planner may want to create two different technologies, say $\theta_{1}$ and $\theta_{2}$, and provide one technology to a subset of firms and the other to the rest. This strategy may be optimal if $C(\theta)$ were sufficiently small (so that duplication costs are not too large).

In the environment outlined here, this option will not typically work because of non-excludability. In partic-
} 
All factor markets are again competitive. Consequently, given the technology offer of $\theta$ of the research firm, the maximization problem of each final good producer is

$$
\max _{\mathbf{Z}^{i} \in \mathcal{Z}, \mathbf{L}^{i} \in \mathcal{L}} \pi\left(\mathbf{Z}^{i}, \mathbf{L}^{i}, \theta\right)=G\left(\mathbf{Z}^{i}, \mathbf{L}^{i}, \theta\right)-\sum_{j=1}^{N} w_{Z j} Z_{j}^{i}-\sum_{j=1}^{M} w_{L j} L_{j}^{i}
$$

Notice the important difference in this maximization problem relative to that in Economy D: firms are only maximizing with respect to $\left(\mathbf{Z}^{i}, \mathbf{L}^{i}\right)$, not with respect to $\theta^{i}$, which will be determined by the research firm.

The objective of the research firm is to maximize total surplus, or total output. Since $\theta^{i}=\theta$ for all $i \in \mathcal{F}$, this is equivalent to

$$
\max _{\theta^{i} \in \Theta} \Pi(\theta)=\int_{0}^{1} G\left(\mathbf{Z}^{i}, \mathbf{L}^{i}, \theta\right) d i-C(\theta) .
$$

This leads to a natural definition of equilibrium:

Definition 2 An equilibrium in Economy $C$ is a set of firm decisions $\left\{\mathbf{Z}^{i}, \mathbf{L}^{i}\right\}_{i \in \mathcal{F}}$, technology choice $\theta$ and factor prices $\left(\mathbf{w}_{Z}, \mathbf{w}_{L}\right)$ such that $\left\{\mathbf{Z}^{i}, \mathbf{L}^{i}\right\}_{i \in \mathcal{F}}$ solve $(7)$ given $\left(\mathbf{w}_{Z}, \mathbf{w}_{L}\right)$ and $\theta$, (3) holds, and the technology choice for the research firm, $\theta$, maximizes (8).

We now impose weaker versions of Assumptions 1 and 1' on $G$ :

Assumption $2 G\left(\mathbf{Z}^{i}, \mathbf{L}^{i}, \theta^{i}\right)$ is jointly strictly concave and increasing in $\left(\mathbf{Z}^{i}, \mathbf{L}^{i}\right)$ and $\mathcal{Z}$ and $\mathcal{L}$ are convex.

Assumption 2' $G\left(\mathbf{Z}^{i}, \mathbf{L}^{i}, \theta^{i}\right)$ is increasing and exhibits constant returns to scale in $\left(\mathbf{Z}^{i}, \mathbf{L}^{i}\right)$, and we have $(\overline{\mathbf{Z}}, \overline{\mathbf{L}}) \in \mathcal{Z} \times \mathcal{L}$.

The important difference between Assumptions 1 and 1' versus Assumptions 2 and 2' is that with the latter, $G\left(\mathbf{Z}^{i}, \mathbf{L}^{i}, \theta^{i}\right)$ does not need to be jointly concave in $\left(\mathbf{Z}^{i}, \theta\right)$, which will play an important role in the results below (nor does $\Theta$ need to be a subset of $\mathbb{R}^{K}$ ).

ular, all firms would want to use the technology that is superior. Nevertheless, there can be some situations in which the research firm may prefer to create two distinct technologies, $\theta_{1}$ and $\theta_{2}$, from the menu. For this, it needs to be the case that first, neither of the two technologies is superior to the other (i.e., which one leads to higher output depends on factor proportions); second, $F$ has to be jointly non-concave in $\theta$ and $(\mathbf{Z}, \mathbf{L})$, so that some firms may choose $\theta_{1}$ and the corresponding factor demand, while others choose $\theta_{2}$ and other levels of factor demands, and all firms make the same level of profits; and third, $C(\theta)$ should be low enough that the costs of creating two different technologies, $\theta_{1}$ and $\theta_{2}$, is not prohibitive. Such situations are relatively rare and are not central to the focus here, so rather than deriving the conditions on $C(\theta)$ and the production function $G$ to rule out this possibility, I simply assume that choosing two separate technologies from the menu is not possible. 
Proposition 2 (Equilibrium Theorem C) Suppose Assumption 2 or Assumption 2' holds. Then any equilibrium technology is a solution to

$$
\max _{\theta \in \Theta} G(\overline{\mathbf{Z}}, \overline{\mathbf{L}}, \theta)-C(\theta)
$$

and any solution to this problem is an equilibrium technology.

Proof. The proof is similar to that of Proposition 1, and follows again by noting that under Assumption 2, the equilibrium will be symmetric, so $\left(\mathbf{Z}^{i}, \mathbf{L}^{i}, \theta\right)=(\mathbf{Z}, \mathbf{L}, \theta)$. In addition, because $G$ is increasing in $\left(\mathbf{Z}^{i}, \mathbf{L}^{i}\right)$, market clearing, (3), yields that $(\mathbf{Z}, \mathbf{L})=(\overline{\mathbf{Z}}, \overline{\mathbf{L}})$, which implies that (8) is identical to (9). When Assumption 2' holds, there are constant returns to scale in $(\mathbf{Z}, \mathbf{L})$, and $(\overline{\mathbf{Z}}, \overline{\mathbf{L}}) \in \mathcal{Z} \times \mathcal{L}$, so we can once again work with a single firm employing $(\overline{\mathbf{Z}}, \overline{\mathbf{L}})$, and the conclusion follows.

Defining $F(\overline{\mathbf{Z}}, \overline{\mathbf{L}}, \theta)=G(\overline{\mathbf{Z}}, \overline{\mathbf{L}}, \theta)-C(\theta)$, we obtain that technology choice in Economy $\mathrm{C}$ can be characterized as maximizing some function $F(\overline{\mathbf{Z}}, \overline{\mathbf{L}}, \theta)$ with respect to $\theta \in \Theta$ as in Economy D. However, I refer to this as an "equilibrium theorem" not as a welfare theorem as for Proposition 1, since despite the fact that the objective of the research firm is to maximize social surplus, the equilibrium may not be the social optimum. This results from the fact that once created, technologies are non-excludable, so all firms use it, whereas the social planner may have preferred to exclude some firms to enable remaining firms to hire more of the factors of production (recall footnote 13). The equilibrium structure, as captured by Definition 2, does not allow for this possibility given the non-excludable nature of the technology.

For our purposes, the more important difference is that while in Economy $\operatorname{D~} F(\overline{\mathbf{Z}}, \overline{\mathbf{L}}, \theta)$ is by assumption jointly concave in $(\mathbf{Z}, \theta)$, the same is not true in Economy C. In particular, in this latter economy, $F(\overline{\mathbf{Z}}, \overline{\mathbf{L}}, \theta)$ does not need to be concave in $(\mathbf{Z}, \theta)$ (nor is it necessarily globally concave in $\theta$ ).

It is also useful to note that equilibrium factor prices are now given by $w_{Z_{j}}=\partial G(\overline{\mathbf{Z}}, \overline{\mathbf{L}}, \theta) / \partial Z_{j}$ and $w_{L j}=\partial G(\overline{\mathbf{Z}}, \overline{\mathbf{L}}, \theta) / \partial L_{j}$, but since $F(\overline{\mathbf{Z}}, \overline{\mathbf{L}}, \theta)=G(\overline{\mathbf{Z}}, \overline{\mathbf{L}}, \theta)-C(\theta)$, this is equivalent to $w_{Z j}=\partial F(\overline{\mathbf{Z}}, \overline{\mathbf{L}}, \theta) / \partial Z_{j}$ and $w_{L j}=\partial F(\overline{\mathbf{Z}}, \overline{\mathbf{L}}, \theta) / \partial L_{j}$ as in Economy D.

\subsection{Economy M-Monopoly Equilibrium}

Now I briefly discuss an economy that is similar to Economy C, but features a monopolist supplying technologies to final good producer firms. I take the simplest structure to deliver results similar to Propositions 1 and 2, while Appendix A analyzes exactly the setup of Economy C with a monopolist provider of technologies. 
In the environment here, there is still a unique final good and each firm has access to the production function

$$
Y^{i}=\alpha^{-\alpha}(1-\alpha)^{-1}\left[G\left(\mathbf{Z}^{i}, \mathbf{L}^{i}, \theta^{i}\right)\right]^{\alpha} q\left(\theta^{i}\right)^{1-\alpha}
$$

which is similar to (6), except that $G\left(\mathbf{Z}^{i}, \mathbf{L}^{i}, \theta^{i}\right)$ is now a subcomponent of the production function, which depends on $\theta^{i}$, the technology being used by the firm. This subcomponent needs to be combined with an intermediate good embodying technology $\theta^{i}$, denoted by $q\left(\theta^{i}\right)$ conditioned on $\theta^{i}$ to emphasize that it embodies technology $\theta^{i}$. This intermediate good is supplied by the monopolist. The term $\alpha^{-\alpha}(1-\alpha)^{-1}$ in the front is a convenient normalization. This structure is a generalization of the setup common in equilibrium models of endogenous technology (e.g., Romer, 1990, Grossman and Helpman, 1991, or Aghion and Howitt, 1992, 1998). As before, I assume that $\mathbf{Z}^{i} \in \mathcal{Z} \subset \mathbb{R}_{+}^{N}, \mathbf{L}^{i} \in \mathcal{L} \subset \mathbb{R}_{+}^{M}$ and $G$ is a real-valued production function that is twice continuously differentiable in $\left(\mathbf{Z}^{i}, \mathbf{L}^{i}\right)$.

The technology monopolist can create technology $\theta$ at $\operatorname{cost} C(\theta)$ from the technology menu, and again I assume that it can only choose one technology. Once created, the technology monopolist can produce as many units of the intermediate good of type $\theta$ (that is, of the intermediate goods embodying technology $\theta$ ) at per unit cost normalized to $1-\alpha$ unit of the final good (this is also a convenient normalization, without any substantive implications). It can then set a (linear) price per unit of the intermediate good of type $\theta$, denoted by $\chi$.

All factor markets are again competitive. Consequently, each firm takes the type of available technology, $\theta$, and the price of the intermediate good embodying this technology, $\chi$, as given and maximizes

$\max _{\substack{\mathbf{Z}^{i} \in \mathcal{Z}, \mathbf{L}^{i} \in \mathcal{L}, q(\theta) \geq 0}} \pi\left(\mathbf{Z}^{i}, \mathbf{L}^{i}, q(\theta) \mid \theta, \chi\right)=\alpha^{-\alpha}(1-\alpha)^{-1}\left[G\left(\mathbf{Z}^{i}, \mathbf{L}^{i}, \theta\right)\right]^{\alpha} q(\theta)^{1-\alpha}-\sum_{j=1}^{N} w_{Z j} Z_{j}^{i}-\sum_{j=1}^{M} w_{L j} L_{j}^{i}-\chi q(\theta)$,

which gives the following simple inverse demand for intermediates of type $\theta$ as a function of its price, $\chi$, and the factor employment levels of the firm as

$$
q^{i}\left(\theta, \chi, \mathbf{Z}^{i}, \mathbf{L}^{i}\right)=\alpha^{-1} G\left(\mathbf{Z}^{i}, \mathbf{L}^{i}, \theta\right) \chi^{-1 / \alpha}
$$

The problem of the monopolist is to maximize its profits (which are equal to price minus marginal cost of production times total sales of the intermediates, minus the cost of creating the technology). Thus the problem of the monopolist is:

$$
\max _{\theta, \chi,\left[q^{i}\left(\theta, \chi, \mathbf{Z}^{i}, \mathbf{L}^{i}\right)\right]_{i \in \mathcal{F}}} \Pi=(\chi-(1-\alpha)) \int_{i \in \mathcal{F}} q^{i}\left(\theta, \chi, \mathbf{Z}^{i}, \mathbf{L}^{i}\right) d i-C(\theta)
$$


subject to (12). Therefore, an equilibrium in this economy can be defined as:

Definition 3 An equilibrium in Economy $M$ is a set of firm decisions $\left\{\mathbf{Z}^{i}, \mathbf{L}^{i}, q^{i}\left(\theta, \chi, \mathbf{Z}^{i}, \mathbf{L}^{i}\right)\right\}_{i \in \mathcal{F}}$, technology choice $\theta$, and factor prices $\left(\mathbf{w}_{Z}, \mathbf{w}_{L}, \chi\right)$ such that $\left\{\mathbf{Z}^{i}, \mathbf{L}^{i}, q^{i}\left(\theta, \chi, \mathbf{Z}^{i}, \mathbf{L}^{i}\right)\right\}_{i \in \mathcal{F}}$ solve (11) given $\left(\mathbf{w}_{Z}, \mathbf{w}_{L}, \chi\right)$ and technology $\theta$, (3) holds, and the technology choice and pricing decision for the monopolist, $(\theta, \chi)$, maximize (13) subject to (12).

Once again the important distinction between this definition and Definition 1 is that now factor demands and technology are being decided by different agents (the former by the final good producers, the latter by the technology monopolist).

The equilibrium in this economy is straightforward to characterize because (12) defines a constant elasticity demand curve, so the optimal price of the monopolist that maximizes (13) is simply the standard monopoly markup, i.e., $1 /(1-\alpha)$ times the marginal cost of production of the intermediate, $1-\alpha$. This leads to an equilibrium monopoly price of $\chi=1$. Moreover, I continue to impose Assumption 2 or 2' (which now apply to $G$, the subcomponent of the production function (10)). Under these assumptions, the equilibrium will again be symmetric, so $q^{i}(\theta, \chi)=\alpha^{-1} G(\overline{\mathbf{Z}}, \overline{\mathbf{L}}, \theta) \chi^{-1 / \alpha}$ for all $i \in \mathcal{F}$, and given the monopoly price $\chi=1$, we have $q^{i}(\theta)=q^{i}(\theta, \chi=1, \overline{\mathbf{Z}}, \overline{\mathbf{L}})=\alpha^{-1} G(\overline{\mathbf{Z}}, \overline{\mathbf{L}}, \theta)$ for all $i \in \mathcal{F}$. The profits and the maximization problem of the monopolist can then be expressed as

$$
\max _{\theta \in \Theta} \Pi(\theta)=G(\overline{\mathbf{Z}}, \overline{\mathbf{L}}, \theta)-C(\theta)
$$

Thus we have established (proof in the text):

Proposition 3 (Equilibrium Theorem $\boldsymbol{M}$ ) Suppose Assumption 2 or Assumption 2' holds. Then an equilibrium in Economy $\mathrm{M}$ is a solution to

$$
\max _{\theta \in \Theta} G(\overline{\mathbf{Z}}, \overline{\mathbf{L}}, \theta)-C(\theta)
$$

and any solution to this problem is an equilibrium.

Relative to Economies D and C, the presence of the monopoly markup implies the presence of greater distortions in this economy. ${ }^{14}$ More important for our purposes here, however, is that again defining $F(\overline{\mathbf{Z}}, \overline{\mathbf{L}}, \theta)=G(\overline{\mathbf{Z}}, \overline{\mathbf{L}}, \theta)-C(\theta)$, equilibrium technology in Economy $\mathrm{M}$ is a solution to a problem identical to that in Economy $\mathrm{C}$, and quite similar to the one in Economy

\footnotetext{
${ }^{14}$ For example, it can be verified that taking the behavior of the final good producers as given, the socially optimal allocation in this case would maximize $(1-\alpha)^{-1 / \alpha}\left[G\left(\overline{\mathbf{Z}}, \overline{\mathbf{L}}, \theta^{i}\right)\right]-C(\theta)$ rather than $\left[G\left(\overline{\mathbf{Z}}, \overline{\mathbf{L}}, \theta^{i}\right)\right]-C(\theta)$.
} 
D. As in Economy C, $F(\overline{\mathbf{Z}}, \overline{\mathbf{L}}, \theta)$ need not be globally concave in $\theta$ nor even locally concave in $(\mathbf{Z}, \theta)$ in the neighborhood of the equilibrium.

This result therefore shows that for the analysis of equilibrium bias, it is not important whether technology choices are at the firm level or at the centralized level (resulting from some $\mathrm{R} \& \mathrm{D}$ or other research process), and also whether they are made to maximize social surplus or monopoly profits. But we will see that whether $F(\overline{\mathbf{Z}}, \overline{\mathbf{L}}, \theta)$ is jointly concave in $(\mathbf{Z}, \theta)$ will play an important role in the results.

Finally, it can be verified that in this economy equilibrium factor prices are given by $w_{Z j}=$ $(1-\alpha)^{-1} \partial G(\overline{\mathbf{Z}}, \overline{\mathbf{L}}, \theta) / \partial Z_{j}$ and $w_{L j}=(1-\alpha)^{-1} \partial G(\overline{\mathbf{Z}}, \overline{\mathbf{L}}, \theta) / \partial L_{j}$, which are proportional to the derivatives of the $F$ function defined as $F(\overline{\mathbf{Z}}, \overline{\mathbf{L}}, \theta)=G(\overline{\mathbf{Z}}, \overline{\mathbf{L}}, \theta)-C(\theta)$. So to facilitate comparison with Economies D and $\mathrm{C}$, with a slight abuse of terminology I will refer to the derivatives of the $F$ function as the "equilibrium factor prices" even in Economy M.

\section{Relative Equilibrium Bias}

The previous section established that in three different environments, with different market structures and conceptions of technology choice, the characterization of equilibrium technology boils down to an identical maximization problem - the maximization of some function $F(\overline{\mathbf{Z}}, \overline{\mathbf{L}}, \theta)$ where $\overline{\mathbf{Z}}$ and $\overline{\mathbf{L}}$ are the factor supplies in the economy. In this and the next two sections, I make use of this characterization to derive a number of results about equilibrium bias of technology choice.

This section analyzes relative equilibrium bias, and for that reason, throughout I focus on a more specialized economy with only two factors, $L$ and $Z$ (i.e., $M=1$ and $N=1$ ), and $\theta \in \Theta \subset \mathbb{R}^{K}$ for some $K \geq 1$, so that $F: \mathbb{R}_{+} \times \mathbb{R}_{+} \times \mathbb{R}^{K} \rightarrow \mathbb{R}_{+}$. Moreover, suppose that $Z \in \mathcal{Z} \subset \mathbb{R}_{+}, L \in \mathcal{L} \subset \mathbb{R}_{+}$, and that $\Theta$ is a convex compact subset of $\mathbb{R}^{K}$ with the $j$ th component denoted by $\theta_{j}$. Finally, I assume that $F$ is twice continuously differentiable in $(Z, L, \theta)$.

Recall that, in a two-factor economy, relative equilibrium bias is defined as the effect of technology on the marginal product (price) of a factor relative to the marginal product (price) of the other factor. Denote the marginal product/price of the two factors by

$$
w_{Z}(Z, L, \theta)=\frac{\partial F(Z, L, \theta)}{\partial Z} \text { and } w_{L}(Z, L, \theta)=\frac{\partial F(Z, L, \theta)}{\partial L},
$$

when employment levels (factor proportions) are given by $(Z, L)$ and the technology is $\theta \cdot{ }^{15}$

\footnotetext{
${ }^{15}$ Recall that $F(\overline{\mathbf{L}}, \overline{\mathbf{Z}}, \theta)$ either corresponds to the production function of the firms (Economy D) or we have
} 
From the twice differentiability of $F$, these marginal products are also differentiable functions of $Z$ and $L$. Then we have the following definitions: ${ }^{16}$

Definition 4 An increase in technology $\theta_{j}$ for some $j=1, \ldots, K$ is relatively biased towards factor $Z$ at $(\bar{Z}, \bar{L}, \theta) \in \mathcal{Z} \times \mathcal{L} \times \Theta$ if

$$
\frac{\partial w_{Z}(\bar{Z}, \bar{L}, \theta) / w_{L}(\bar{Z}, \bar{L}, \theta)}{\partial \theta_{j}} \geq 0 .
$$

This definition simply expresses what it means for a technology to be relatively biased towards a factor (similarly a decrease in $\theta_{j}$ is relatively biased towards factor $Z$, if the derivative in Definition 4 is non-positive). From this definition, it is clear that (weak) relative equilibrium bias should correspond to a change in technology $\theta$ in a direction biased towards $Z$ in response to an increase in $\bar{Z}$ (or $\bar{Z} / \bar{L}$ ); this is stated in the next definition. ${ }^{17}$

Definition 5 Denote the equilibrium technology at factor supplies $(\bar{Z}, \bar{L}) \in \mathcal{Z} \times \mathcal{L}$ by $\theta(\bar{Z}, \bar{L})$, and assume that $\partial \theta_{j}(\bar{Z}, \bar{L}) / \partial Z$ exists at $(\bar{Z}, \bar{L})$ for all for all $j=1, \ldots, K$. Then there is relative equilibrium bias at $(\bar{Z}, \bar{L}, \theta(\bar{Z}, \bar{L}))$ if

$$
\sum_{j=1}^{K} \frac{\partial w_{Z}(\bar{Z}, \bar{L}, \theta(\bar{Z}, \bar{L})) / w_{L}(\bar{Z}, \bar{L}, \theta(\bar{Z}, \bar{L}))}{\partial \theta_{j}} \frac{\partial \theta_{j}(\bar{Z}, \bar{L})}{\partial Z} \geq 0 .
$$

Notice that the definition of relative equilibrium bias requires the (overall) change in technology in response to an increase in $\bar{Z}$ to be biased towards $Z$ at the point $(\bar{Z}, \bar{L}) \in \mathcal{Z} \times \mathcal{L}$ for which $\partial \theta_{j}(\bar{Z}, \bar{L}) / \partial Z$ exists for all $j$. The statement is not qualified with "towards $Z$ " since relative equilibrium bias is also equivalent to a decline in $Z$ inducing a change in technology relatively biased against $Z$. Finally, the requirement that $\partial \theta_{j}(\bar{Z}, \bar{L}) / \partial Z$ exists for all $j$ used in this definition will be further discussed below (in particular, see the discussion after Theorem 3 in the next section).

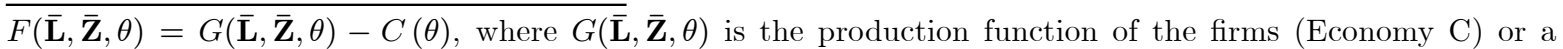
subcomponent of the production function (Economy M). In both cases, the derivatives of $F$ with respect to $\mathbf{Z}$ and $\mathbf{L}$ define the marginal products of these factors. With a slight abuse of terminology, I will refer to $F(\overline{\mathbf{L}}, \overline{\mathbf{Z}}, \theta)$ as "the production function".

${ }^{16}$ For this section, all definitions are "local" in the sense that, I will only look at the effect of small changes in factor supplies. This is why they are expressed in terms of derivatives. I do not add this qualification to simplify terminology.

${ }^{17}$ Throughout this section, I focus on changes in the supply of factor $Z$, which is also equivalent to a change in relative supplies $Z / L$ (with $L$ kept constant). Moreover, I denote the change in equilibrium technology by $\partial \theta_{j}(\bar{Z}, \bar{L}) / \partial Z$ rather than $d \theta_{j}(\bar{Z}, \bar{L}) / d Z$ since $\theta$ is not generally only a function of $Z$. I reserve the notation $d\left(w_{Z} / w_{L}\right) / d Z$ to denote the total change in relative (or absolute) wages, which includes the technological adjustment, and contrast this with the partial change $\partial\left(w_{Z} / w_{L}\right) / \partial Z$, which holds technology constant (see, for example, (16)). 
The next definition introduces the more stringent concept of strong relative bias, which requires that in response to an increase in $\bar{Z}$, technology changes so much that the overall effect (after the induced change in technology) is to increase the relative price of factor $Z$.

Definition 6 Denote the equilibrium technology at factor supplies $(\bar{Z}, \bar{L}) \in \mathcal{Z} \times \mathcal{L}$ by $\theta(\bar{Z}, \bar{L})$, and assume that $\partial \theta_{j}(\bar{Z}, \bar{L}) / \partial Z$ exists at $(\bar{Z}, \bar{L})$ for all $j=1, \ldots, K$. Then there is strong relative equilibrium bias at $(\bar{Z}, \bar{L}, \theta(\bar{Z}, \bar{L}))$ if

$$
\begin{aligned}
\frac{d w_{Z}(\bar{Z}, \bar{L}, \theta(\bar{Z}, \bar{L})) / w_{L}(\bar{Z}, \bar{L}, \theta(\bar{Z}, \bar{L}))}{d Z} & =\frac{\partial w_{Z}(\bar{Z}, \bar{L}, \theta(\bar{Z}, \bar{L})) / w_{L}(\bar{Z}, \bar{L}, \theta(\bar{Z}, \bar{L}))}{\partial Z}+ \\
& \sum_{j=1}^{K} \frac{\partial w_{Z}(\bar{Z}, \bar{L}, \theta(\bar{Z}, \bar{L})) / w_{L}(\bar{Z}, \bar{L}, \theta(\bar{Z}, \bar{L}))}{\partial \theta_{j}} \frac{\partial \theta_{j}(\bar{Z}, \bar{L})}{\partial Z}>0 .
\end{aligned}
$$

By comparing the latter two definitions, it is clear that there will be strong relative equilibrium bias if the sum of the expressions in (15) over $j=1, \ldots, K$ is large enough to dominate the direct (negative) effect of the increase in relative supplies on relative wages (which is the first term in (16)).

The main result in this section is that the conjecture about relative equilibrium bias applies in a world with only factor-augmenting technologies, but not more generally. Before deriving these results, it is useful to clarify the notions introduced so far using an example, which captures the main findings in Acemoglu (1998, 2002), but in the context of Economy C or M studied above rather than in the models of the original papers. In particular, the next example considers an environment equivalent to Economy $\mathrm{C}$ or $\mathrm{M}$ above, with constant returns to scale in $L$ and $Z$. This example will also clarify one possible meaning of the Hicks's quote discussed above (see in particular footnote 20).

Example 1 (Relative Equilibrium Bias) Suppose that

$$
G(Z, L, \theta)=\left[\gamma\left(A_{Z} Z\right)^{\frac{\sigma-1}{\sigma}}+(1-\gamma)\left(A_{L} L\right)^{\frac{\sigma-1}{\sigma}}\right]^{\frac{\sigma}{\sigma-1}}
$$

where $\theta=\left(A_{Z}, A_{L}\right)$. In particular, $A_{Z}$ and $A_{L}$ are two separate factor-augmenting technology terms, $\gamma \in(0,1)$ is a distribution parameter which determines how important the two factors are, and $\sigma \in(0, \infty)$ is the elasticity of substitution between the two factors. When $\sigma=\infty$, the two factors are perfect substitutes, and the production function is linear. When $\sigma=1$, the production function is Cobb-Douglas, and when $\sigma=0$, there is no substitution between the two factors, and the production function is Leontieff. Since there are two technology terms, I take $\theta=\left(A_{Z}, A_{L}\right) \in \Theta=\mathbb{R}_{+}^{2}$. 
Suppose that factor supplies are given by $(\bar{Z}, \bar{L})$. Then the relative marginal product of the two factors is:

$$
\frac{w_{Z}}{w_{L}}=\frac{\gamma}{1-\gamma}\left(\frac{A_{Z}}{A_{L}}\right)^{\frac{\sigma-1}{\sigma}}\left(\frac{\bar{Z}}{\bar{L}}\right)^{-\frac{1}{\sigma}} .
$$

The relative marginal product of $Z$ is decreasing in the relative abundance of $Z, \bar{Z} / \bar{L}$. This is the usual substitution effect, leading to a downward-sloping relative demand curve. This expression also makes it clear that the measure of relative bias towards $Z$ will correspond to $\bar{\theta}=\left(A_{Z} / A_{L}\right)^{(\sigma-1) / \sigma}{ }^{18}$ since higher levels of $\bar{\theta}$ increase the marginal product of $Z$ relative to labor for all values of $\sigma$ (recall Definition 4). To derive the results similar to those in Acemoglu $(1998,2002)$ in the context of Economy C or M, suppose that the costs of producing new technologies are $\eta_{Z} A_{Z}^{1+\delta}$ and $\eta_{L} A_{L}^{1+\delta}$, where $\delta>0$. Despite the fact that $\delta>0$ introduces diminishing returns in the choice of technology, the production possibilities set of this economy is nonconvex, since there is choice both over the factors of production, $Z$ and $L$, and the technologies, $A_{Z}$ and $A_{L}$ (so that the function (17) exhibits increasing returns in $L, Z, A_{Z}$ and $A_{L}$ ). From Proposition 2 or 3 , the problem of choosing equilibrium technology is the following strictly concave maximization problem:

$$
\max _{A_{Z}, A_{L}}\left[\gamma\left(A_{Z} \bar{Z}\right)^{\frac{\sigma-1}{\sigma}}+(1-\gamma)\left(A_{L} \bar{L}\right)^{\frac{\sigma-1}{\sigma}}\right]^{\frac{\sigma}{\sigma-1}}-\eta_{Z} A_{Z}^{1+\delta}-\eta_{L} A_{L}^{1+\delta} .
$$

Taking the ratio of the first-order necessary and sufficient conditions with respect to $A_{Z}$ and $A_{L}$, and denoting the equilibrium values by ${ }^{*}$ 's, the solution to this problem yields

$$
\frac{A_{Z}^{*}}{A_{L}^{*}}=\left(\frac{\eta_{Z}}{\eta_{L}}\right)^{-\frac{\sigma}{1+\sigma \delta}}\left(\frac{\gamma}{1-\gamma}\right)^{\frac{\sigma}{1+\sigma \delta}}\left(\frac{\bar{Z}}{\bar{L}}\right)^{\frac{\sigma-1}{1+\sigma \delta}} .
$$

This equation can also be expressed in an alternative form, both useful for the discussion here and for Theorem 1 below:

$$
\frac{\partial \ln \left(A_{Z}^{*} / A_{L}^{*}\right)}{\partial \ln (Z / L)}=\frac{\sigma-1}{1+\sigma \delta} .
$$

Recall that there will be weak equilibrium bias, if the expression in Definition 4 is nonnegative. Using equation (18), $w_{Z}\left(\bar{Z}, \bar{L}, A_{Z}^{*}, A_{L}^{*}\right) / w_{L}\left(\bar{Z}, \bar{L}, A_{Z}^{*}, A_{L}^{*}\right)$ is a function of $A_{Z}^{*} / A_{L}^{*}$ rather than $A_{Z}^{*}$ and $A_{Z}^{*}$ separately. Using this (and preparing for Theorem 1), we can express the condition

\footnotetext{
${ }^{18}$ Alternatively, we could define $A_{Z}^{(\sigma-1) / \sigma}$ and $A_{L}^{-(\sigma-1) / \sigma}$ as two separate technology terms, both relatively biased towards $Z$, but clearly focusing on $\bar{\theta}$ is more economical.

It is important that the bias towards factor $Z$ is $\bar{\theta}=\left(A_{Z} / A_{L}\right)^{(\sigma-1) / \sigma}$, not $A_{Z} / A_{L}$, as is sometimes confusingly and incorrectly stated in the applied literature. $A_{Z} / A_{L}$ is the ratio of $Z$-augmenting to $L$-augmenting technology. When $\sigma>1$, an increase in $A_{Z} / A_{L}$ increases the relative marginal product of $Z$, while when $\sigma<1$, an increase in $A_{Z} / A_{L}$ reduces the relative marginal product of $Z$.
} 
for weak relative equilibrium bias as: ${ }^{19}$

$$
\Delta\left(w_{Z} / w_{L}\right) \equiv \frac{\partial \ln \left(w_{Z}\left(\bar{Z}, \bar{L}, A_{Z}^{*}, A_{L}^{*}\right) / w_{L}\left(\bar{Z}, \bar{L}, A_{Z}^{*}, A_{L}^{*}\right)\right)}{\partial \ln \left(A_{Z} / A_{L}\right)} \frac{\partial \ln \left(A_{Z}^{*} / A_{L}^{*}\right)}{\partial \ln (Z / L)} \geq 0 .
$$

Using (18) and (20),

$$
\Delta\left(w_{Z} / w_{L}\right)=\frac{\sigma-1}{\sigma} \times \frac{\sigma-1}{1+\sigma \delta}=\frac{(\sigma-1)^{2}}{(1+\sigma \delta) \sigma} \geq 0
$$

which is always nonnegative, thus establishing that there is always weak relative equilibrium bias (as claimed by the conjecture in the Introduction). Alternatively, the same result follows by looking directly at the measure of relative bias towards $Z$ introduced above, $\bar{\theta}=\left(A_{Z} / A_{L}\right)^{(\sigma-1) / \sigma}$. In particular, substituting for (19), we have

$$
\bar{\theta}=\left(\frac{\eta_{Z}}{\eta_{L}}\right)^{-\frac{\sigma-1}{1+\sigma \delta}}\left(\frac{\gamma}{1-\gamma}\right)^{\frac{\sigma-1}{1+\sigma \delta}}\left(\frac{\bar{Z}}{\bar{L}}\right)^{\frac{(\sigma-1)^{2}}{(1+\sigma \delta) \sigma}}
$$

which is always increasing in $\bar{Z} / \bar{L} .^{20}$

Next to investigate the conditions under which there is strong relative equilibrium bias, we can use Definition 6 and check the conditions for (again using log derivatives for simplicity):

$$
\begin{aligned}
& \frac{\partial \ln \left(w_{Z}\left(\bar{Z}, \bar{L}, A_{Z}^{*}, A_{L}^{*}\right) / w_{L}\left(\bar{Z}, \bar{L}, A_{Z}^{*}, A_{L}^{*}\right)\right)}{\partial \ln (Z / L)} \\
& +\frac{\partial \ln \left(w_{Z}\left(\bar{Z}, \bar{L}, A_{Z}^{*}, A_{L}^{*}\right) / w_{L}\left(\bar{Z}, \bar{L}, A_{Z}^{*}, A_{L}^{*}\right)\right)}{\partial \ln \left(A_{Z}^{*} / A_{L}^{*}\right)} \frac{\partial \ln \left(A_{Z}^{*} / A_{L}^{*}\right)}{\partial \ln (Z / L)}>0 .
\end{aligned}
$$

From (18) and (21), this condition is equivalent to

$$
-\frac{1}{\sigma}+\frac{(\sigma-1)^{2}}{(1+\sigma \delta) \sigma}=\frac{\sigma-2-\delta}{1+\sigma \delta}>0
$$

\footnotetext{
${ }^{19}$ Expressing everything in terms of log changes rather than absolute changes is simply for convenience (and also useful for Theorem 1). In particular, note that as long as $x>0$ and $a>0, \partial x / \partial a \gtreqless 0$ if and only if $\partial \ln x / \partial \ln a \gtreqless 0$. Moreover, since $L$ is kept constant, $\partial x / \partial(Z / L)=(\partial x / \partial Z) L$, so whenever the expression here is nonnegative so is the expression in Definition 4; i.e., $\Delta\left(w_{Z} / w_{L}\right) \geq 0$ if and only if $\sum_{j=1}^{K} \partial w_{Z}(\bar{Z}, \bar{L}, \theta(\bar{Z}, \bar{L})) / w_{L}(\bar{Z}, \bar{L}, \theta(\bar{Z}, \bar{L})) / \partial \theta_{j} \times \partial \theta_{j}(\bar{Z}, \bar{L}) / \partial Z \geq 0$.

${ }^{20}$ More explicitly, returning to the discussion in footnote 18 , when $\sigma>1$, an increase in $\bar{Z} / \bar{L}$ increases $A_{Z} / A_{L}$, which in turn raises $w_{Z} / w_{L}$ at given factor proportions. In contrast when $\sigma<1$, an increase in $\bar{Z} / \bar{L}$ reduces $A_{Z} / A_{L}$, but in this case, $A_{Z} / A_{L}$ is relatively biased against factor $Z$ (it is biased towards factor $L$ ), so a decrease in $A_{Z} / A_{L}$ again raises $w_{Z} / w_{L}$.

At this point, we can also return to Hicks' quote. This example (and the theorem that follows) show that the claim in Hicks' quote is not true for relative bias of technical change; an increase in the abundance of a factor that reduces its price will make technology relatively biased towards that factor. However, if we interpret the quote in terms of relative factor-augmenting changes, equation (20) shows that it is true when $\sigma<1$. Therefore, one interpretation of Hicks' claim is that the increase in the abundance of a factor will cause technology relatively augmenting the other factor as long as the elasticity of substitution between the two factors is less than one.
} 
so that when $\sigma>2+\delta$, the relative demand curve for factors is upward-sloping and there is strong relative equilibrium bias. In this example, this result can be obtained more transparently by substituting for $A_{Z}^{*} / A_{L}^{*}$ from (19) into (18) to obtain:

$$
\frac{w_{Z}}{w_{L}}=\left(\frac{\eta_{Z}}{\eta_{L}}\right)^{-\frac{\sigma-1}{1+\sigma \delta}}\left(\frac{\gamma}{1-\gamma}\right)^{\frac{\sigma+\sigma \delta}{1+\sigma \delta}}\left(\frac{\bar{Z}}{\bar{L}}\right)^{\frac{\sigma-2-\delta}{1+\sigma \delta}}
$$

and thus

$$
\frac{d \ln \left(w_{Z}\left(\bar{Z}, \bar{L}, A_{Z}^{*}, A_{L}^{*}\right) / w_{L}\left(\bar{Z}, \bar{L}, A_{Z}^{*}, A_{L}^{*}\right)\right)}{d \ln (Z / L)}=\frac{\sigma-2-\delta}{1+\sigma \delta},
$$

which confirms the result of strong relative equilibrium bias when $\sigma>2+\delta$ shown in (22). ${ }^{21}$

This example therefore illustrates the possibility of both weak and strong relative bias results in an economy with a nonconvex aggregate production possibilities set. In particular, technological change induced in response to an increase in $Z$ is always (weakly) relatively biased towards $Z$, and moreover, if the condition $\sigma>2+\delta$ is satisfied, there is also strong relative bias. This example also corresponds to the most general result that exists in the literature (see Acemoglu, 2002). Nevertheless, the structure of the economy is quite special, in particular, it incorporates a specific aggregate production function and cost functions for undertaking research. I next establish that with a more general setup, but still with two-factors and factor-augmenting technologies, the same results hold. Before stating this theorem, recall that a function $f(x, y)$ is homothetic in $x$ and $y$, if $(\partial f(x, y) / \partial x) /(\partial f(x, y) / \partial y)$ is only a function of $x / y$ for all $x$ and $y$.

Theorem 1 (Relative Equilibrium Bias with Factor-Augmenting Technologies) Consider Economy $\mathrm{C}$ or $\mathrm{M}$ with two-factors, $(Z, L) \in \mathcal{Z} \times \mathcal{L} \subset \mathbb{R}_{+}^{2}$, and two factor-augmenting technologies, $\left(A_{Z}, A_{L}\right) \in \mathbb{R}_{+}^{2}$, such that the production function is $F\left(A_{Z} Z, A_{L} L\right)$. Assume that $F$ is twice continuously differentiable, concave and homothetic in its two arguments, and that the costs of producing technologies $A_{Z}$ and $A_{L}, C\left(A_{Z}, A_{L}\right)$, is also twice continuously differentiable, strictly convex and homothetic in $A_{Z}$ and $A_{L}$. Denote the first derivatives of $C\left(A_{Z}, A_{L}\right)$ by $C_{Z}$ and $C_{L}$. Let $\sigma$ be the (local) elasticity of substitution between $Z$ and $L$ defined by $\sigma=-\left.\frac{\partial \ln (Z / L)}{\partial \ln \left(w_{Z} / w_{L}\right)}\right|_{\frac{A_{Z}}{A_{L}}}$, and let $\delta=\frac{\partial \ln \left(C_{Z}\left(A_{Z}, A_{L}\right) / C_{L}\left(A_{Z}, A_{L}\right)\right)}{\partial \ln \left(A_{Z} / A_{L}\right)}$. Finally, suppose that

\footnotetext{
${ }^{21}$ In Acemoglu (2002), the condition for upward-sloping relative demand curves was $\sigma>2-\delta^{\prime}$ for some other parameter $\delta^{\prime}>0$, which essentially corresponds to $-\delta$ here. The reason is that in that context, as in many endogenous growth models, the technology allowed for knowledge-spillovers, and the parameter $\delta^{\prime}$ measured how much a particular type of technology benefits from past innovations in the same line, adding another degree of non-convexity. Here a higher value of the parameter $\delta$ makes the aggregate technology of the economy more "convex" and thus upward-sloping relative demand curves less likely. See also Theorem 8 below.
} 
factor supplies are given by $(\bar{Z}, \bar{L})$ and denote equilibrium technologies by $\left(A_{Z}^{*}, A_{L}^{*}\right)$, and equilibrium factor prices by $w_{Z}\left(\bar{Z}, \bar{L}, A_{Z}^{*}, A_{L}^{*}\right)$ and $w_{L}\left(\bar{Z}, \bar{L}, A_{Z}^{*}, A_{L}^{*}\right)$. Then we have that for all $(\bar{Z}, \bar{L}) \in \mathcal{Z} \times \mathcal{L}:$

$$
\frac{\partial \ln \left(A_{Z}^{*} / A_{L}^{*}\right)}{\partial \ln (Z / L)}=\frac{\sigma-1}{1+\sigma \delta}
$$

and

$$
\frac{\partial \ln \left(w_{Z}\left(\bar{Z}, \bar{L}, A_{Z}^{*}, A_{L}^{*}\right) / w_{L}\left(\bar{Z}, \bar{L}, A_{Z}^{*}, A_{L}^{*}\right)\right)}{\partial \ln \left(A_{Z} / A_{L}\right)} \frac{\partial \ln \left(A_{Z}^{*} / A_{L}^{*}\right)}{\partial \ln (Z / L)} \geq 0
$$

so that there is always weak relative equilibrium bias. Moreover,

$$
\frac{d \ln \left(w_{Z}\left(\bar{Z}, \bar{L}, A_{Z}^{*}, A_{L}^{*}\right) / w_{L}\left(\bar{Z}, \bar{L}, A_{Z}^{*}, A_{L}^{*}\right)\right)}{d \ln (Z / L)}=\frac{\sigma-2-\delta}{1+\sigma \delta},
$$

so that there is strong relative equilibrium bias if $\sigma-2-\delta>0$.

Proof. By Proposition 2 or 3, we need to look at the following strictly concave maximization problem:

$$
\max _{A_{L}, A_{Z}} F\left(A_{Z} \bar{Z}, A_{L} \bar{L}\right)-C\left(A_{Z}, A_{L}\right) .
$$

Taking the ratio of the first-order necessary and sufficient conditions gives

$$
\overline{\bar{Z}} \frac{F_{Z}\left(A_{Z}^{*} \bar{Z}, A_{L}^{*} \bar{L}\right)}{F_{L}\left(A_{Z}^{*} \bar{Z}, A_{L}^{*} \bar{L}\right)}=\frac{C_{Z}\left(A_{Z}^{*}, A_{L}^{*}\right)}{C_{L}\left(A_{Z}^{*}, A_{L}^{*}\right)}
$$

where $F_{Z}$ denotes the derivative of $F$ with respect to its first argument and $F_{L}$ denotes the derivative with respect to the second, and $C_{Z}$ and $C_{L}$ are defined as in the theorem. Recalling the definition of marginal products, this gives

$$
\frac{\bar{Z}}{\bar{L}} \frac{w_{Z}\left(\bar{Z}, \bar{L}, A_{Z}^{*}, A_{L}^{*}\right)}{w_{L}\left(\bar{Z}, \bar{L}, A_{Z}^{*}, A_{L}^{*}\right)}=\frac{\bar{Z}}{\bar{L}} \frac{A_{Z}^{*}}{A_{L}^{*}} \frac{F_{Z}\left(A_{Z}^{*} \bar{Z}, A_{L}^{*} \bar{L}\right)}{F_{L}\left(A_{Z}^{*} \bar{Z}, A_{L}^{*} \bar{L}\right)}=\frac{A_{Z}^{*}}{A_{L}^{*}} \frac{C_{Z}\left(A_{Z}^{*}, A_{L}^{*}\right)}{C_{L}\left(A_{Z}^{*}, A_{L}^{*}\right)} .
$$

Now since $F$ is homothetic, $F_{Z} / F_{L}$ is only a function of $Z / L$ and $A_{Z} / A_{L}$. Moreover, since $C$ is homothetic, $C_{Z} / C_{L}$ is also only a function of $A_{Z} / A_{L}$ and $\delta$ in the theorem is well defined. Using these facts, taking logs in (27) and differentiating totally with respect to $\ln (Z / L)$ gives:

$$
\begin{aligned}
\left(1+\frac{\partial \ln \left(C_{Z}\left(A_{Z}^{*}, A_{L}^{*}\right) / C_{L}\left(A_{Z}^{*}, A_{L}^{*}\right)\right)}{\partial \ln \left(A_{Z} / A_{L}\right)}\right) \frac{\partial \ln \left(A_{Z}^{*} / A_{L}^{*}\right)}{\partial \ln (Z / L)}= & \left.\frac{\partial \ln \left(w_{Z} / w_{L}\right)}{\partial \ln (Z / L)}\right|_{\frac{A_{Z}^{*}}{A_{L}^{*}}}+1 \\
& +\left.\frac{\partial \ln \left(w_{Z} / w_{L}\right)}{\partial \ln \left(A_{Z} / A_{L}\right)}\right|_{\frac{\bar{Z}}{L}} \frac{\partial \ln \left(A_{Z}^{*} / A_{L}^{*}\right)}{\partial \ln (Z / L)} .
\end{aligned}
$$

Equation (27) and the definition of $\sigma$ yield:

$$
\frac{\partial \ln \left(w_{Z} / w_{L}\right)}{\partial \ln \left(A_{Z} / A_{L}\right)}=\frac{\sigma-1}{\sigma} .
$$


Substituting (29) into (28), rearranging and recalling the definitions of $\delta$ and $\sigma$, we obtain

$$
\frac{\partial \ln \left(A_{Z}^{*} / A_{L}^{*}\right)}{\partial \ln (Z / L)}=\frac{\sigma-1}{1+\sigma \delta}
$$

as in (24). Then (25) immediately follows by combining this with (29), and by the same argument as in Example 1 (in particular footnote 19), this establishes weak equilibrium bias.

Finally, (26) and strong equilibrium bias (under the condition that $\sigma>2+\delta$ ) follow from (24) by noting that

$$
\begin{aligned}
\frac{d \ln \left(w_{Z}\left(\bar{Z}, \bar{L}, A_{Z}^{*}, A_{L}^{*}\right) / w_{L}\left(\bar{Z}, \bar{L}, A_{Z}^{*}, A_{L}^{*}\right)\right)}{d \ln (Z / L)} & =-\frac{1}{\sigma}+\frac{\sigma-1}{\sigma} \frac{\partial \ln \left(A_{Z}^{*} / A_{L}^{*}\right)}{\partial \ln (Z / L)} \\
& =\frac{\sigma-2-\delta}{1+\sigma \delta} .
\end{aligned}
$$

The major result of this theorem is that the insights from Example 1 generalize in a fairly natural way as long as the potential menu of technological possibilities only consists of two technologies, one augmenting $Z$ and the other $L{ }^{22}$ The only difference is that the parameter $\delta$ and the elasticity of substitution $\sigma$ are no longer constants, but are functions of $A_{L}, A_{Z}$, $\bar{L}$ and $\bar{Z}$, so changes in factor supplies will have effects that depend on the local elasticity of substitution and the local value of $\delta$. Nevertheless, the change in $A_{Z} / A_{L}$ (or in $\left(A_{Z} / A_{L}\right)^{(\sigma-1) / \sigma}$ as in Example 1) induced by an increase in $\bar{Z}$ is always relatively biased towards $Z$, and there is strong equilibrium relative bias if $\sigma>2+\delta$. Therefore, this theorem establishes that an environment with a menu of technological possibilities featuring only factor-augmenting technologies is sufficient to obtain both a general weak relative bias theorem, and the possibility of strong relative bias (when the local elasticity of substitution between factors, $\sigma$, is sufficiently high and the parameter $\delta$ is relatively low).

However, once we depart from the world of Theorem 1 with the production function $F\left(A_{Z} Z, A_{L} L\right)$, it is possible for the supply of factor $Z$ to increase, and in response, technology to change in a direction relatively biased against this factor (i.e., towards factor $L$ ), thus disproving the conjecture in the Introduction. This is stated in the next theorem and proved by providing two counterexamples.

Theorem 2 With a general menu of technologies, there is not necessarily relative equilibrium bias. That is, suppose that $\partial \theta_{j}(\bar{Z}, \bar{L}) / \partial Z$ exists at $(\bar{Z}, \bar{L})$ for all $j=1, \ldots, K$, then

$$
\sum_{j=1}^{K} \frac{\partial w_{Z}(\bar{Z}, \bar{L}, \theta(\bar{Z}, \bar{L})) / w_{L}(\bar{Z}, \bar{L}, \theta(\bar{Z}, \bar{L}))}{\partial \theta_{j}} \frac{\partial \theta_{j}(\bar{Z}, \bar{L})}{\partial Z}<0
$$

\footnotetext{
${ }^{22}$ The additional assumptions are that $F$ and $C$ are homothetic.
} 
is possible.

I will present two counterexamples highlighting different reasons for why there is no such general relative bias theorem. For simplicity, both of the examples focus on economies with a single dimension of technology. The first one shows that a choice over the elasticity of substitution may lead to endogenous technological change biased against the factor that is becoming more abundant. ${ }^{23}$ The second one shows that the form of the production function in Theorem $1, F\left(A_{Z} Z, A_{L} L\right)$, is important for the result.

Example 2 (Counterexample I) Consider an example of Economy $\mathrm{C}$ or $\mathrm{M}$ with the family of production functions satisfying Assumption 2':

$$
G(Z, L, \theta)=\left[Z^{\theta}+L^{\theta}\right]^{1 / \theta}
$$

and cost of technology creation, $C(\theta)$, defined over $\Theta=[a, b]$ where $b>a$, that is convex and twice continuously differentiable over the entire $\Theta$, with $C^{\prime}$ denoting its first derivative. From Proposition 2 or 3 , the choice of $\theta$ will maximize $F(Z, L, \theta)=G(Z, L, \theta)-C(\theta)$. Therefore, at given factor supplies $(\bar{Z}, \bar{L})$, the equilibrium technology choice $\theta$ satisfies

$\frac{\partial F(\bar{Z}, \bar{L}, \tilde{\theta})}{\partial \theta}=-\frac{1}{\tilde{\theta}^{2}}\left[\bar{Z}^{\tilde{\theta}}+\bar{L}^{\tilde{\theta}}\right]^{1 / \tilde{\theta}} \ln \left[\bar{Z}^{\tilde{\theta}}+\bar{L}^{\tilde{\theta}}\right]+\frac{1}{\tilde{\theta}}\left(\bar{Z}^{\tilde{\theta}} \ln \bar{Z}+\bar{L}^{\tilde{\theta}} \ln \bar{L}\right)\left[\bar{Z}^{\tilde{\theta}}+\bar{L}^{\tilde{\theta}}\right]^{(1-\tilde{\theta}) / \tilde{\theta}}-C^{\prime}(\tilde{\theta})=0$

with $\partial^{2} F(\bar{Z}, \bar{L}, \tilde{\theta}) / \partial \theta^{2}<0$. From Definition 4 , a counterexample would correspond to

$$
\Delta\left(w_{Z} / w_{L}\right) \equiv \frac{\partial w_{Z}(\bar{Z}, \bar{L}, \theta(\bar{Z}, \bar{L})) / w_{L}(\bar{Z}, \bar{L}, \theta(\bar{Z}, \bar{L}))}{\partial \theta} \frac{\partial \theta(\bar{Z}, \bar{L})}{\partial Z}<0 .
$$

From the Implicit Function Theorem, ${ }^{24}$ this is equivalent to

$$
\Delta\left(w_{Z} / w_{L}\right) \equiv-\frac{\partial w_{Z}(\bar{Z}, \bar{L}, \theta(\bar{Z}, \bar{L})) / w_{L}(\bar{Z}, \bar{L}, \theta(\bar{Z}, \bar{L}))}{\partial \theta} \frac{\partial^{2} F(\bar{Z}, \bar{L}, \theta(\bar{Z}, \bar{L})) / \partial \theta \partial Z}{\partial^{2} F(\bar{Z}, \bar{L}, \theta(\bar{Z}, \bar{L})) / \partial \theta^{2}}<0,
$$

or since $\partial^{2} F(\bar{Z}, \bar{L}, \theta(\bar{Z}, \bar{L})) / \partial \theta^{2}<0$, to

$$
\frac{\partial w_{Z}(\bar{Z}, \bar{L}, \theta(\bar{Z}, \bar{L})) / w_{L}(\bar{Z}, \bar{L}, \theta(\bar{Z}, \bar{L}))}{\partial \theta} \frac{\partial^{2} F(\bar{Z}, \bar{L}, \theta(\bar{Z}, \bar{L}))}{\partial \theta \partial Z}<0 .
$$

\footnotetext{
${ }^{23}$ See Benabou (2005) for a model of endogenous choice of the elasticity of substitution as a function of the inequality of human capital among workers in the economy.

${ }^{24}$ See, for example, Rudin (1964), Theorem 9.18, or Simon and Blume (1994), Theorem 15.2.
} 
Now differentiate the expression in (31) with respect to $\bar{Z}$ and to simplify notation denote $\theta(\bar{Z}, \bar{L})$ by $\tilde{\theta}$ :

$$
\begin{aligned}
\frac{\partial^{2} F(\bar{Z}, \bar{L}, \tilde{\theta})}{\partial \theta \partial Z} & =-\frac{1}{\tilde{\theta}^{2}} \bar{Z}^{\tilde{\theta}-1}\left[\bar{Z}^{\tilde{\theta}}+\bar{L}^{\tilde{\theta}}\right]^{\frac{1-\tilde{\theta}}{\tilde{\theta}}} \ln \left[\bar{Z}^{\tilde{\theta}}+\bar{L}^{\tilde{\theta}}\right]-\frac{1}{\tilde{\theta}} \bar{Z}^{\tilde{\theta}-1}\left[\bar{Z}^{\tilde{\theta}}+\bar{L}^{\tilde{\theta}}\right]^{\frac{1-\tilde{\theta}}{\tilde{\theta}}} \\
& +\frac{1-\tilde{\theta}}{\tilde{\theta}} \bar{Z}^{\tilde{\theta}-1}\left(\bar{Z}^{\tilde{\theta}} \ln \bar{Z}+\bar{L}^{\tilde{\theta}} \ln \bar{L}\right)\left[\bar{Z}^{\tilde{\theta}}+\bar{L}^{\tilde{\theta}}\right]^{\frac{1-2 \tilde{\theta}}{\tilde{\theta}}}+\frac{1}{\tilde{\theta}} \bar{Z}^{\tilde{\theta}-1}(\tilde{\theta} \ln \bar{Z}+1)\left[\bar{Z}^{\tilde{\theta}}+\bar{L}^{\tilde{\theta}}\right]^{\frac{1-\tilde{\theta}}{\tilde{\theta}}} \\
& \propto-\frac{1}{\tilde{\theta}} \ln \left[\bar{Z}^{\tilde{\theta}}+\bar{L}^{\tilde{\theta}}\right]-1+(1-\tilde{\theta})\left(\bar{Z}^{\tilde{\theta}} \ln \bar{Z}+\bar{L}^{\tilde{\theta}} \ln \bar{L}\right)\left[\bar{Z}^{\tilde{\theta}}+\bar{L}^{\tilde{\theta}}\right]^{-1}+(\tilde{\theta} \ln \bar{Z}+1)
\end{aligned}
$$

If this expression is negative, then in response to an increase in $\bar{Z}, \tilde{\theta}=\theta(\bar{Z}, \bar{L})$ will decline. Moreover, from (30), we have

$$
\frac{w_{Z}(Z, L, \tilde{\theta})}{w_{L}(Z, L, \tilde{\theta})}=\left(\frac{Z}{L}\right)^{\tilde{\theta}-1}
$$

which is increasing in $\tilde{\theta}$ as long as $Z>L$. Now suppose that we start with $\bar{L}=1$ and $\bar{Z}=2$, and choose the function $C(\theta)$ such that $\tilde{\theta}=0.1$. Then

$$
\begin{aligned}
\frac{\partial^{2} F(\bar{Z}=2, \bar{L}=1, \tilde{\theta}=0.1)}{\partial \theta \partial Z} & \propto-\frac{1}{0.1} \ln \left[1+2^{0.1}\right]-1+0.9\left(2^{0.1} \ln 2\right)\left[1+2^{0.1}\right]^{-1}+(0.1 \ln 2+1) \\
& \propto-7.28-1+0.32+1.07<0
\end{aligned}
$$

which is clearly negative, so

$$
\frac{\partial w_{Z}(\bar{Z}=2, \bar{L}=1, \tilde{\theta}=0.1) / w_{L}(\bar{Z}=2, \bar{L}=1, \tilde{\theta}=0.1)}{\partial \theta} \times \frac{\partial^{2} F(\bar{Z}=2, \bar{L}=1, \tilde{\theta}=0.1)}{\partial \theta \partial Z}<0,
$$

and (33) is satisfied, providing a counterexample to the conjecture. Put differently, in this case the increase in $\bar{Z}$ induces a decline in $\tilde{\theta}$, which is a change in technology relatively biased against $Z$.

Example 3 (Counterexample II) ${ }^{25}$ Consider again an example of Economy $\mathrm{C}$ or $\mathrm{M}$ with a family of production functions satisfying Assumption 2':

$$
G(Z, L, \theta)=Z \theta+L \theta^{2}
$$

and cost of creating new technologies given by $C_{0} \theta^{2} / 2$ with $C_{0}>0$ for all $\theta \in \Theta=\mathbb{R}$ and $L \in \mathcal{L} \subset\left(0, C_{0} / 2\right)$. This production function exhibits constant returns to scale in $Z$ and $L$,

\footnotetext{
${ }^{25}$ I thank Rabah Amir for suggesting an example along these lines.
} 
and $F(Z, L, \theta)=G(Z, L, \theta)-C(\theta)$ is strictly concave in $\theta$ for $L \in \mathcal{L}$. The optimal choice of $\theta$ satisfies

$$
\theta(\bar{Z}, \bar{L})=\frac{\bar{Z}}{C_{0}-2 \bar{L}}
$$

and is thus increasing in $\bar{Z}$ for $\bar{L} \in \mathcal{L}$. Relative price of the two factors is given by: $w_{Z}(\theta) / w_{L}(\theta)=$ $\theta^{-1}$, which is clearly decreasing in $\theta$. Therefore, an increase in $\bar{Z}$ will induce technological change (or technology adoption) relatively biased against $Z$. Theorem 1 does not apply in this case since the production function does not take the form $F\left(A_{Z} Z, A_{L} L\right)$, even though it is homothetic in $Z$ and $L$.

Theorem 1 explains the reason for the negative result in Theorem 2. The conjecture about relative bias does not apply in these examples because the menu of technologies does not take this simple form with one technology augmenting factor $Z$ and the other factor $L$. Although this type of factor-augmenting technology may be an interesting and empirically important special case, one may be interested in a more general theorem that applies without imposing a specific structure on the interaction between technologies and the factors of production. This is especially the case when we consider technology choices that correspond to shifts from one type of organizational form or organization of production to another, such as those experienced during recent decades, during the emergence of the American System of Manufacturing, or during the Industrial Revolution. These shifts not only change the productivity of different factors, but the way the whole production process is organized and thus naturally also the elasticity of substitution between factors.

Examples 2 and 3 show that a general theorem is not possible for relative bias. Nevertheless, it is also important to emphasize that these examples and Theorem 2 do not imply that with the general menu of technologies, changes in relative supplies will cause technical change that it is relatively biased against the more abundant factor. In many cases, weak equilibrium bias will still apply, but without imposing more structure, we do not have a general theorem.

In the next section, we will see that such a theorem can be derived for absolute bias. In fact, Examples 2 and 3 already hint at this possibility. The reason why induced technology (in response to an increase in $\bar{Z}$ ) is not relatively biased towards $Z$ in both examples is that the induced change in technology increases $w_{Z}$ (at given factor proportions), but it has an even larger (positive) effect on the marginal product of the other factor, $w_{L} \cdot{ }^{26}$

\footnotetext{
${ }^{26}$ To see this more explicitly, note that $\partial^{2} F(\bar{Z}, \bar{L}, \theta(\bar{Z}, \bar{L})) / \partial \theta \partial Z=\partial w_{Z}(\bar{Z}, \bar{L}, \theta(\bar{Z}, \bar{L})) / \partial \theta$ and rewrite
} 


\section{Equilibrium Absolute Bias}

Examples 2 and 3 show that there is no general theorem about relative equilibrium bias unless we restrict ourselves to factor-augmenting technologies. The obvious question is whether there is a general result for absolute bias. The answer is yes and is the focus of this section. Recall that absolute bias refers to whether new technology increases the marginal product of a factor. The main results in this section will therefore show that in response to increases in the supply of a factor (or a set of factors), technology will change endogenously in a direction absolutely biased towards this factor (or this set of factors).

As stated in the Introduction, this section focuses on weak (absolute) bias results and presents both local and global theorems. I begin with the local theorem, which applies to the case with $N=1$, i.e., to changes in the supply of a single factor, $Z$.

Given the results in Section 2, the problem of equilibrium technology choice is again equivalent to

$$
\max _{\theta \in \Theta} F(\bar{Z}, \overline{\mathbf{L}}, \theta)
$$

where $\overline{\mathbf{L}}$ denotes the supply of these other inputs and $\bar{Z}$ denotes the supply of $Z$. Let us denote the marginal product (or price) of this factor by $w_{Z}(\bar{Z}, \overline{\mathbf{L}}, \theta)=\partial F(\bar{Z}, \overline{\mathbf{L}}, \theta) / \partial Z$ when the employment levels of factors are given by $(\bar{Z}, \overline{\mathbf{L}})$ and the technology is $\theta$. For the local result I will also take $\Theta$ to be a convex compact subset of $\mathbb{R}^{K}$ for some $K \geq 1$ and assume that $F$ is also twice differentiable in $(Z, \theta)$, which implies that $w_{Z}(\bar{Z}, \overline{\mathbf{L}}, \theta)$ is differentiable in $\theta$.

Definition 7 Let $\theta \in \Theta \subset \mathbb{R}^{K}$. An increase in technology $\theta_{j}$ for some $j=1, \ldots, K$ is locally absolutely biased towards factor $Z$ at $(\bar{Z}, \overline{\mathbf{L}}) \in \mathcal{Z} \times \mathcal{L}$ if

$$
\frac{\partial w_{Z}(\bar{Z}, \overline{\mathbf{L}}, \theta)}{\partial \theta_{j}} \geq 0 .
$$

Conversely we could define a decrease in technology $\theta$ as locally absolutely biased towards factor $Z$ if the same derivative is nonpositive. Notice also that the local bias definition requires the bias for only small changes in technology and only at the current factor proportions $(\bar{Z}, \overline{\mathbf{L}})$.

equation $(32)$ as

$$
\Delta\left(w_{Z} / w_{L}\right) \equiv-\frac{\partial w_{Z}(\bar{Z}, \bar{L}, \theta(\bar{Z}, \bar{L})) / w_{L}(\bar{Z}, \bar{L}, \theta(\bar{Z}, \bar{L}))}{\partial \theta} \frac{\partial w_{Z}(\bar{Z}, \bar{L}, \theta(\bar{Z}, \bar{L})) / \partial \theta}{\partial^{2} F(\bar{Z}, \bar{L}, \theta(\bar{Z}, \bar{L})) / \partial \theta^{2}} .
$$

When $w_{L}(\bar{Z}, \bar{L}, \theta(\bar{Z}, \bar{L}))$ is constant, this is equivalent to equation (40) in the proof of Theorem 3 in the next section and is always nonnegative. However, as Example 2 and 3 show, a large effect of $\theta$ on $w_{L}$ can reverse this result. 
The global definition below will require a similar directional change but for all magnitudes of changes in supplies and at all factor proportions. Next we define (local) equilibrium absolute bias analogously to relative equilibrium bias.

Definition 8 Let $\theta \in \Theta \subset \mathbb{R}^{K}$. Denote the equilibrium technology at factor supplies $(\bar{Z}, \overline{\mathbf{L}}) \in$ $\mathcal{Z} \times \mathcal{L}$ by $\theta(\bar{Z}, \overline{\mathbf{L}})$ and assume that $\partial \theta_{j}(\bar{Z}, \overline{\mathbf{L}}) / \partial Z$ exists at $(\bar{Z}, \overline{\mathbf{L}})$ for all $j=1, \ldots, K$. Then there is local absolute equilibrium bias at $(\bar{Z}, \overline{\mathbf{L}}, \theta(\bar{Z}, \overline{\mathbf{L}}))$ if

$$
\sum_{j=1}^{K} \frac{\partial w_{Z}(\bar{Z}, \overline{\mathbf{L}}, \theta(\bar{Z}, \overline{\mathbf{L}}))}{\partial \theta_{j}} \frac{\partial \theta_{j}(\bar{Z}, \overline{\mathbf{L}})}{\partial Z} \geq 0 .
$$

In words, this definition requires the induced combined change in the components of technology resulting from an increase in $\bar{Z}$ to be towards increasing the marginal product of factor $Z$. As in Definition 5 for relative equilibrium bias, this definition also requires $\partial \theta_{j}(\bar{Z}, \overline{\mathbf{L}}) / \partial Z$ to exist for all $j$. The next theorem will also be stated under this assumption, which can alternatively be replaced by Assumption A1 below.

Theorem 3 (Local Absolute Bias) Consider Economy D, C or M. Suppose that $\Theta$ is a convex subset of $\mathbb{R}^{K}$ and $F(Z, \mathbf{L}, \theta)$ is twice continuously differentiable in $(Z, \theta)$. Let the equilibrium technology at factor supplies $(\bar{Z}, \overline{\mathbf{L}})$ be $\theta(\bar{Z}, \overline{\mathbf{L}})$, and assume that $\theta(\bar{Z}, \overline{\mathbf{L}})$ is in the interior of $\Theta$ and that $\partial \theta_{j}(\bar{Z}, \overline{\mathbf{L}}) / \partial Z$ exists at $(\bar{Z}, \overline{\mathbf{L}})$ for all $j=1, \ldots, K$. Then, there is local absolute equilibrium bias at all $(\bar{Z}, \overline{\mathbf{L}}) \in \mathcal{Z} \times \mathcal{L}$, i.e.,

$$
\sum_{j=1}^{K} \frac{\partial w_{Z}(\bar{Z}, \overline{\mathbf{L}}, \theta(\bar{Z}, \overline{\mathbf{L}}))}{\partial \theta_{j}} \frac{\partial \theta_{j}(\bar{Z}, \overline{\mathbf{L}})}{\partial Z} \geq 0 \text { for all }(\bar{Z}, \overline{\mathbf{L}}) \in \mathcal{Z} \times \mathcal{L} .
$$

Moreover, if $\partial \theta_{j}(\bar{Z}, \overline{\mathbf{L}}) / \partial Z \neq 0$ for some $j=1, \ldots, K$, then

$$
\sum_{j=1}^{K} \frac{\partial w_{Z}(\bar{Z}, \overline{\mathbf{L}}, \theta(\bar{Z}, \overline{\mathbf{L}}))}{\partial \theta_{j}} \frac{\partial \theta_{j}(\bar{Z}, \overline{\mathbf{L}})}{\partial Z}>0 .
$$

Proof. The proof follows from the Implicit Function Theorem. For expositional clarity, I first present the case where $\theta \in \Theta \subset \mathbb{R}$. Since $\Theta \subset \mathbb{R}$ and by hypothesis, the equilibrium choice of $\theta$ is in the interior of $\Theta$, we have

$$
\frac{\partial F(\bar{Z}, \overline{\mathbf{L}}, \theta(\bar{Z}, \overline{\mathbf{L}}))}{\partial \theta}=0
$$

and $\partial^{2} F(\bar{Z}, \overline{\mathbf{L}}, \theta(\bar{Z}, \overline{\mathbf{L}})) / \partial \theta^{2} \leq 0$. Since $\partial \theta(\bar{Z}, \overline{\mathbf{L}}) / \partial Z$ exists at $(\bar{Z}, \overline{\mathbf{L}})$ by hypothesis, from the Implicit Function Theorem it must be equal to

$$
\frac{\partial \theta(\bar{Z}, \overline{\mathbf{L}})}{\partial Z}=-\frac{\partial^{2} F(\bar{Z}, \overline{\mathbf{L}}, \theta(\bar{Z}, \overline{\mathbf{L}})) / \partial \theta \partial Z}{\partial^{2} F(\bar{Z}, \overline{\mathbf{L}}, \theta(\bar{Z}, \overline{\mathbf{L}})) / \partial \theta^{2}}=-\frac{\partial w_{Z}(\bar{Z}, \overline{\mathbf{L}}, \theta(\bar{Z}, \overline{\mathbf{L}})) / \partial \theta}{\partial^{2} F(\bar{Z}, \overline{\mathbf{L}}, \theta(\bar{Z}, \overline{\mathbf{L}})) / \partial \theta^{2}}
$$


so we must have $\partial^{2} F(\bar{Z}, \overline{\mathbf{L}}, \theta(\bar{Z}, \overline{\mathbf{L}})) / \partial \theta^{2} \neq 0$, i.e., $\partial^{2} F(\bar{Z}, \overline{\mathbf{L}}, \theta(\bar{Z}, \overline{\mathbf{L}})) / \partial \theta^{2}<0$. This in turn implies:

$$
\frac{\partial w_{Z}(\bar{Z}, \overline{\mathbf{L}}, \theta(\bar{Z}, \overline{\mathbf{L}}))}{\partial \theta} \frac{\partial \theta(\bar{Z}, \overline{\mathbf{L}})}{\partial Z}=-\frac{\left[\partial w_{Z}(\bar{Z}, \overline{\mathbf{L}}, \theta(\bar{Z}, \overline{\mathbf{L}})) / \partial \theta\right]^{2}}{\partial^{2} F(\bar{Z}, \overline{\mathbf{L}}, \theta(\bar{Z}, \overline{\mathbf{L}})) / \partial \theta^{2}} \geq 0
$$

establishing (36). Moreover, if $\partial \theta(\bar{Z}, \overline{\mathbf{L}}) / \partial Z \neq 0$, then from $(39), \partial w_{Z}(\bar{Z}, \overline{\mathbf{L}}, \theta(\bar{Z}, \overline{\mathbf{L}})) / \partial \theta \neq$ 0 , so (40) holds with strict inequality, establishing (37).

Next, let us look at the general case where $\theta \in \Theta \subset \mathbb{R}^{K}$ with $K>1$. Let $\Delta w_{Z}$ be the change in $w_{Z}$ resulting from the induced change in $\theta$ (at given factor proportions) as in equation $(35)$ :

$$
\Delta w_{Z}=\sum_{j=1}^{K} \frac{\partial w_{Z}(\bar{Z}, \overline{\mathbf{L}}, \theta(\bar{Z}, \overline{\mathbf{L}}))}{\partial \theta_{j}} \frac{\partial \theta_{j}(\bar{Z}, \overline{\mathbf{L}})}{\partial Z}
$$

Then, we have that

$$
\begin{aligned}
\Delta w_{Z} & =\left[\nabla_{\theta} w_{Z}(\bar{Z}, \overline{\mathbf{L}}, \theta(\bar{Z}, \overline{\mathbf{L}}))\right]^{\prime}\left[\nabla_{Z} \theta(\bar{Z}, \overline{\mathbf{L}})\right] \\
& =\left[\nabla_{\theta Z}^{2} F(\bar{Z}, \overline{\mathbf{L}}, \theta(\bar{Z}, \overline{\mathbf{L}}))\right]^{\prime}\left[\nabla_{Z} \theta(\bar{Z}, \overline{\mathbf{L}})\right],
\end{aligned}
$$

where $\left[\nabla_{\theta} w_{Z}(\bar{Z}, \overline{\mathbf{L}}, \theta(\bar{Z}, \overline{\mathbf{L}}))\right]$ is a $K \times 1$ vector of changes in $w_{Z}$ in response to each component of $\theta \in \Theta \subset \mathbb{R}^{K},\left[\nabla_{Z} \theta(\bar{Z}, \overline{\mathbf{L}})\right]$ is the Jacobian of $\theta$ with respect to $Z$, i.e., a $K \times 1$ vector of changes in each component of $\theta$ in response to the change in $\bar{Z}$, and for a matrix (vector) $v, v^{\prime}$ denotes its transpose. The second line in (41) uses the fact that $w_{Z}$ is the derivative of the $F$ function, so $\left[\nabla_{\theta Z}^{2} F(\bar{Z}, \overline{\mathbf{L}}, \theta(\bar{Z}, \overline{\mathbf{L}}))\right]$ is also the $K \times 1$ vector of changes in $w_{Z}$ in response to each component of $\theta$. Since $\partial \theta_{j}(\bar{Z}, \overline{\mathbf{L}}) / \partial Z$ exists at $(\bar{Z}, \overline{\mathbf{L}})$ for all $j$, the gradient $\nabla_{Z} \theta(\bar{Z}, \overline{\mathbf{L}})$ also exists and from the Implicit Function Theorem, it satisfies

$$
\nabla_{Z} \theta(\bar{Z}, \overline{\mathbf{L}})^{\prime}=-\left[\nabla_{\theta Z}^{2} F(\bar{Z}, \overline{\mathbf{L}}, \theta(\bar{Z}, \overline{\mathbf{L}}))\right]^{\prime}\left[\nabla_{\theta \theta}^{2} F(\bar{Z}, \overline{\mathbf{L}}, \theta(\bar{Z}, \overline{\mathbf{L}}))\right]^{-1},
$$

where $\nabla_{\theta \theta}^{2} F(\bar{Z}, \overline{\mathbf{L}}, \theta(\bar{Z}, \overline{\mathbf{L}}))$ is the $K \times K$ Hessian of $F$ with respect to $\theta$. The fact that $\theta(\bar{Z}, \overline{\mathbf{L}})$ is a solution to the maximization problem (34) implies that $\nabla_{\theta \theta}^{2} F(\bar{Z}, \overline{\mathbf{L}}, \theta(\bar{Z}, \overline{\mathbf{L}}))$ is negative semi-definite. That $\nabla_{Z} \theta(\bar{Z}, \overline{\mathbf{L}})$ exists then implies that $\nabla_{\theta \theta}^{2} F(\bar{Z}, \overline{\mathbf{L}}, \theta(\bar{Z}, \overline{\mathbf{L}}))$ is nonsingular, so it must be negative definite. Since it is a Hessian, it is also symmetric. Therefore, its inverse $\left[\nabla_{\theta \theta}^{2} F(\bar{Z}, \overline{\mathbf{L}}, \theta(\bar{Z}, \overline{\mathbf{L}}))\right]^{-1}$ is also symmetric and negative definite. Substituting in (41), we obtain

$$
\Delta w_{Z}=-\left[\nabla_{\theta Z}^{2} F(\bar{Z}, \overline{\mathbf{L}}, \theta(\bar{Z}, \overline{\mathbf{L}}))\right]^{\prime}\left[\nabla_{\theta \theta}^{2} F(\bar{Z}, \overline{\mathbf{L}}, \theta(\bar{Z}, \overline{\mathbf{L}}))\right]^{-1}\left[\nabla_{\theta Z}^{2} F(\bar{Z}, \overline{\mathbf{L}}, \theta(\bar{Z}, \overline{\mathbf{L}}))\right] \geq 0
$$

which establishes (36) for the case in which $\Theta \subset \mathbb{R}^{K}$. 
By the definition of a negative definite matrix $B, x^{\prime} B x<0$ for all $x \neq 0$, so to establish the strict inequality in (37) in this case, it suffices that one element of $\nabla_{Z} \theta(\bar{Z}, \overline{\mathbf{L}})$ be non-zero, i.e., $\partial \theta_{j}(\bar{Z}, \overline{\mathbf{L}}) / \partial Z \neq 0$ for one $j=1, \ldots K$, completing the proof.

This theorem therefore shows that once we shift our focus to absolute bias, there is a fairly general result. Under minimal assumptions (further discussed below), technological change induced by a change in factor supplies will be biased towards the factor that has become more abundant. There is a clear parallel here with the LeChatelier principle of Samuelson (1947), but also a number of important differences. First, this theorem concerns how marginal products (or prices) change as a result of induced technological changes resulting from changes in factor supplies rather than the elasticity of short-run and long-run demand curves. Second, it applies to the equilibrium of an economy not to the maximization problem of a single firm. Nevertheless, the parallel is also important, since we can think of the change in technology as happening in the "long run", in which case Theorem 3 states that long-run changes in marginal products (factor prices) will be less than those in the short run because of induced technological change or technology adoption.

Theorem 3 was stated and proved under the assumption that $\partial \theta_{j}(\bar{Z}, \overline{\mathbf{L}}) / \partial Z$ exists at $(\bar{Z}, \overline{\mathbf{L}})$ for all $j=1, \ldots, K$. This assumption entails two restrictions. The first is the usual non-singularity requirement to enable an application of the Implicit Function Theorem, i.e., that the Hessian of $F$ with respect to $\theta, \nabla_{\theta \theta}^{2} F(\bar{Z}, \bar{L}, \theta(\bar{Z}, \bar{L}))$, is non-singular at the point $\theta(\bar{Z}, \bar{L})$ (see, for example, Rudin, 1964, Theorem 9.18, or Simon and Blume, 1994, Theorem 15.2; recall also the argument in the proof of Theorem 3). The second is more subtle; since we have not made global concavity assumptions (except in Economy D), a small change in $Z$ may shift the technology choice from one local optimum to another, thus essentially making $\partial \theta_{j}(\bar{Z}, \bar{L}) / \partial Z$ infinite (or undefined). This possibility is also ruled out by this assumption. In fact, the assumption that $\partial \theta_{j}(\bar{Z}, \bar{L}) / \partial Z$ exists at $(\bar{Z}, \bar{L})$ can be replaced by the following:

Assumption A1: $\nabla_{\theta \theta}^{2} F(\bar{Z}, \bar{L}, \theta(\bar{Z}, \bar{L}))$ is non-singular, and there exists $\xi>0$ such that for all $\theta^{\prime} \in \Theta$ with $\partial F\left(\bar{Z}, \bar{L}, \theta^{\prime}\right) / \partial \theta=0$ and $\theta^{\prime} \neq \theta(\bar{Z}, \bar{L})$, we have $F(\bar{Z}, \bar{L}, \theta(\bar{Z}, \bar{L}))$ $F\left(\bar{Z}, \bar{L}, \theta^{\prime}\right)>\xi$.

The second part of the assumption ensures that the peaks of the function $F(\bar{Z}, \bar{L}, \theta)$ in $\theta$ are "well separated", in the sense that in response to a small change in factor supplies, there will not be a shift in the global optimum of $\theta$ from one local optimum to another. ${ }^{27}$

\footnotetext{
${ }^{27}$ Put differently, suppose that the equilibrium maximization problem (34) has multiple local maxima, and
} 
Consequently, Assumption A1 is equivalent to assuming that $\partial \theta_{j}(\bar{Z}, \bar{L}) / \partial Z$ exists at $(\bar{Z}, \bar{L})$ for all $j$. A straightforward condition to ensure that Assumption A1 is satisfied is to assume that $F$ is strictly quasi-concave in $\theta$, though this is considerably stronger than Assumption A1. Since it is more intuitive to directly assume that the derivatives $\partial \theta_{j}(\bar{Z}, \overline{\mathbf{L}}) / \partial Z$ 's exist rather than imposing Assumption A1, I state the relevant theorems under this direct assumption. But depending on taste, Assumption A1 can be substituted in Theorem 3 and some of the subsequent theorems.

Three shortcomings of Theorem 3 are apparent. First, it applies to changes in the supply of a single factor. Second, it applies only to local (small) changes. Third, and perhaps most importantly, as Definition 8 makes it clear, equilibrium bias is a local phenomenon. For example, an increase in $\bar{Z}$ may change $\theta(\bar{Z}, \overline{\mathbf{L}})$ in a direction biased towards $Z$ at factor proportions $(\bar{Z}, \overline{\mathbf{L}})$, but this change may in fact be biased against $Z$ at some different factor proportions, say $\left(\bar{Z}^{\prime}, \overline{\mathbf{L}}^{\prime}\right)$. Similar to Milgrom and Roberts' (1996) generalization of LeChatelier principle, there is a global version of Theorem 3 , which will strengthen and generalize it to deal with all of these problems. ${ }^{28}$ Like the results in Milgrom and Roberts (1996), this theorem also uses tools from the theory of monotone comparative statics. I start with changes in a single factor, and then generalize it to multiple factors.

Definition 9 Let $\theta(\bar{Z}, \overline{\mathbf{L}})$ be the equilibrium technology choice in an economy with factor supplies $(\bar{Z}, \overline{\mathbf{L}})$. We say that there is global absolute equilibrium bias if for any $\bar{Z}^{\prime}, \bar{Z} \in \mathcal{Z}$,

$$
\bar{Z}^{\prime} \geq \bar{Z} \Longrightarrow w_{Z}\left(\tilde{Z}, \overline{\mathbf{L}}, \theta\left(\bar{Z}^{\prime}, \overline{\mathbf{L}}\right)\right) \geq w_{Z}(\tilde{Z}, \overline{\mathbf{L}}, \theta(\bar{Z}, \overline{\mathbf{L}})) \text { for all } \tilde{Z} \in \mathcal{Z} \text { and } \overline{\mathbf{L}} \in \mathcal{L} \text {. }
$$

Note that there are two notions of "globality" in this definition. First, the increase from $\bar{Z}$ to $\bar{Z}^{\prime}$ is not limited to small changes. Second, the change in technology induced by this increase is required to increase the price of factor $Z$ for all $\tilde{Z} \in \mathcal{Z}$. Once again, this definition can be made stronger by requiring strict inequality.

denote the set of these maxima at factor proportions $(\bar{Z}, \bar{L})$ by $\Theta^{s}(\bar{Z}, \bar{L})$. All of these solutions satisfy the first-order necessary conditions of the equilibrium maximization problem (34). Suppose $\hat{\theta}(\bar{Z}, \bar{L})$ is a vector that satisfies these first-order necessary conditions. Given the non-singularity assumption (first part of Assumption A1), the Implicit Function Theorem can be applied to $\hat{\theta}(\bar{Z}, \bar{L})$. However, this does not guarantee that $\partial \theta(\bar{Z}, \bar{L}) / \partial Z$ exists, since $\theta(\bar{Z}, \bar{L})$ corresponds to the global maximum of $(34)$, and the change in $Z$ may shift the global maximum from $\hat{\theta}(\bar{Z}, \bar{L})$ to some other $\tilde{\theta}(\bar{Z}, \bar{L}) \in \Theta^{s}(\bar{Z}, \bar{L})$. The second part of Assumption A1 rules this possibility out by imposing that one of the solutions to the first-order necessary conditions gives uniformly higher value, so that a small (infinitesimal) change in $Z$ cannot induce a shift from one element of $\Theta^{s}(\bar{Z}, \bar{L})$ to another.

${ }^{28} \mathrm{~A}$ fourth potential shortcoming is that Theorem 3 is stated assuming that $\theta(\bar{Z}, \overline{\mathbf{L}})$ is in the interior of $\Theta$. This is straightforward to relax. Nevertheless, since the global theorem, Theorem 4, naturally covers the case in which $\theta(\bar{Z}, \overline{\mathbf{L}})$ may be at the boundary of $\Theta$, I do not introduce the additional notation to deal with this case in Theorem 3 (see also the proof of Theorem 6). 
To state the main results, we need a number of technical definitions. Appendix B defines lattices, supermodular functions and (strictly) increasing differences. Both supermodularity and increasing differences loosely correspond to the notion of complementarities, i.e., the requirement that a change in a function resulting from an increase in one argument is itself increasing in the other arguments. In the case of continuously differentiable functions, we have a particularly simple definition of supermodularity (see, e.g., Topkis, 1998):

Definition 10 Let $x=\left(x_{1}, \ldots, x_{n}\right)$ be a vector in $X \subset \mathbb{R}^{n}$, and suppose that the real-valued function $f(x)$ is twice continuously differentiable in $x$. Then $f(x)$ is supermodular on $X$ if and only if $\partial^{2} f(x) / \partial x_{i} \partial x_{i^{\prime}} \geq 0$ for all $x \in X$ and for all $i \neq i^{\prime}$.

Increasing differences is a slightly weaker concept again related to complementarities:

Definition 11 Let $X$ and $T$ be partially ordered sets. Then a function $f(x, t)$ defined on a subset $S$ of $X \times T$ has increasing differences in $(x, t)$, if for all $t^{\prime \prime}>t, f\left(x, t^{\prime \prime}\right)-f(x, t)$ is nondecreasing in $x$. Moreover, $f(x, t)$ has strictly increasing differences in $(x, t)$, if for all $t^{\prime \prime}>t, f\left(x, t^{\prime \prime}\right)-f(x, t)$ is strictly increasing in $x$.

Lemma 2 in Appendix B shows that (strict) supermodularity in $(x, t)$ implies (strict) increasing differences in $(x, t)$. With these definitions, we can use Topkis' Monotonicity Theorem, given as Theorem 10 in Appendix B. Using this approach, we now have:

Theorem 4 (Global Equilibrium Bias) Consider Economy D, C or M. Suppose that $\Theta$ is a lattice, let $\overline{\mathcal{Z}}$ be the convex hull of $\mathcal{Z}$, let $\theta(\bar{Z}, \overline{\mathbf{L}})$ be the equilibrium technology at factor proportions $(\bar{Z}, \overline{\mathbf{L}})$, and suppose that $F(Z, \mathbf{L}, \theta)$ is continuously differentiable in $Z$, supermodular in $\theta$ on $\Theta$ for all $Z \in \overline{\mathcal{Z}}$ and $\mathbf{L} \in \mathcal{L}$, and exhibits strictly increasing differences in $(Z, \theta)$ on $\overline{\mathcal{Z}} \times \Theta$ for all $\mathbf{L} \in \mathcal{L}$, then there is global absolute equilibrium bias, i.e., for any $\bar{Z}^{\prime}, \bar{Z} \in \mathcal{Z}, \bar{Z}^{\prime} \geq \bar{Z}$ implies

$$
\theta\left(\bar{Z}^{\prime}, \overline{\mathbf{L}}\right) \geq \theta(\bar{Z}, \overline{\mathbf{L}}) \text { for all } \overline{\mathbf{L}} \in \mathcal{L}
$$

and

$$
w_{Z}\left(\tilde{Z}, \overline{\mathbf{L}}, \theta\left(\bar{Z}^{\prime}, \overline{\mathbf{L}}\right)\right) \geq w_{Z}(\tilde{Z}, \overline{\mathbf{L}}, \theta(\bar{Z}, \overline{\mathbf{L}})) \text { for all } \tilde{Z} \in \mathcal{Z} \text { and } \overline{\mathbf{L}} \in \mathcal{L} \text {. }
$$

Proof. The proof follows from the application of Theorem 10 in Appendix B. Given the continuity and the supermodularity of $F(Z, \mathbf{L}, \theta)$ on $\overline{\mathcal{Z}} \times \Theta$ and the fact that $\Theta$ is a lattice and $\mathcal{Z}$ is a subset of $\mathbb{R}$ therefore also a lattice, Theorem 10 in Appendix $\mathrm{B}$ implies that 
the set of equilibrium technologies is a non-empty, compact and complete sublattice of $\Theta$. Moreover, supermodularity of $F$ in $\theta$ and strict increasing differences in $(Z, \theta)$ implies that $\bar{Z}^{\prime} \geq \bar{Z} \Longrightarrow \theta\left(\bar{Z}^{\prime}, \overline{\mathbf{L}}\right) \geq \theta(\bar{Z}, \overline{\mathbf{L}})$ for all $\overline{\mathbf{L}} \in \mathcal{L}$. Next (strict) increasing differences of $F(Z, \mathbf{L}, \theta)$ in $(Z, \theta)$ on $\overline{\mathcal{Z}} \times \Theta$ implies that $\partial F(\tilde{Z}, \overline{\mathbf{L}}, \theta) / \partial Z$ is increasing in $\theta$ for all $\tilde{Z} \in\left[\bar{Z}, \bar{Z}^{\prime}\right] \subset \overline{\mathcal{Z}}$. Since $w_{Z}\left(\tilde{Z}, \overline{\mathbf{L}}, \theta\left(\bar{Z}^{\prime}, \overline{\mathbf{L}}\right)\right)=\partial F\left(\tilde{Z}, \overline{\mathbf{L}}, \theta\left(\bar{Z}^{\prime}, \overline{\mathbf{L}}\right)\right) / \partial Z$, the conclusion follows.

An important feature of this theorem, as opposed to the local theorem, Theorem 3 , is that consistent with Definition 9, the induced change in technology does not only increase the marginal product of factor $Z$ (which is becoming more abundant) at the current supply, $\bar{Z}$, but does so at all points in the set $\mathcal{Z}$. In this sense, Theorem 4 is indeed a global theorem, applying both for large magnitudes of changes and applying to all admissible levels of factor supplies. $^{29}$

Also in this theorem, the fact that $\theta\left(\bar{Z}^{\prime}, \overline{\mathbf{L}}\right) \geq \theta(\bar{Z}, \overline{\mathbf{L}})$ (say rather than $\theta\left(\bar{Z}^{\prime}, \overline{\mathbf{L}}\right) \leq$ $\theta(\bar{Z}, \overline{\mathbf{L}}))$ is not particularly important, since the order over the set $\Theta$ is not specified. It could be that as $\bar{Z}$ increases some measure of technology $t$ declines. But in this case, this measure would correspond to a type of technology biased against factor $Z$. If so, we can simply change the order over this parameter, e.g., we can consider changes in $\tilde{t}=-t$ rather than $t$.

Remark 1 Inspection of Theorem 10 in Appendix B will show that Theorem 4 also applies, when the assumption that $F$ is supermodular in $\theta$ is replaced with the weaker assumption that $F$ is quasi-supermodular in $\theta$, which is an ordinal property introduced by Milgrom and Shannon (1994). But Example 4 below shows that (strict) increasing differences cannot be replaced with the (strict) single crossing property of Milgrom and Shannon (1994). Thus, interestingly, Theorem 4 requires a mixture of ordinal and cardinal conditions. Nevertheless, I stated the result under the stronger assumption of supermodularity since quasi-supermodularity is only defined in Appendix B.

Remark 2 Theorem 4 can also be stated assuming only increasing differences in $(Z, \theta)$ rather than strict increasing differences. But in this case, the conclusion that $\theta\left(\bar{Z}^{\prime}, \overline{\mathbf{L}}\right) \geq \theta(\bar{Z}, \overline{\mathbf{L}})$ would only apply to the greatest and the least elements of the set of equilibrium technologies (see Theorem 2.8.1 in Topkis, 1998). The greatest and the least elements of the maximizer set always exist since, given the assumptions of Theorem 4, Theorem 10 ensures that the maximizer

\footnotetext{
${ }^{29}$ It is also useful to note that Theorem 3 could be derived from Theorem 4 by restricting the set $\Theta$ to an arbitrarily small ball around $\theta(\bar{Z}, \overline{\mathbf{L}})$ and then taking a second-order Taylor approximation to $F$ as in Corollary to Theorem 2 of Milgrom and Roberts (1996, p. 176).
} 
set is a complete lattice. Given this result, in the discussion I will simplify the terminology by often referring to increasing differences rather than strict increasing differences.

Two immediate related corollaries of this theorem are also useful to note. Both of those strengthen the results of the theorem to obtain strict inequalities. The first states that this is the case whenever $\theta\left(\bar{Z}^{\prime}, \overline{\mathbf{L}}\right)>\theta(\bar{Z}, \overline{\mathbf{L}})$ (thus is similar to the result on strict inequalities in Theorem 3), while the second imposes additional conditions to ensure $\theta\left(\bar{Z}^{\prime}, \overline{\mathbf{L}}\right)>\theta(\bar{Z}, \overline{\mathbf{L}})$.

Corollary 1 Suppose that the hypotheses in Theorem 4 hold. If in addition $\theta\left(\bar{Z}^{\prime}, \overline{\mathbf{L}}\right) \neq$ $\theta(\bar{Z}, \overline{\mathbf{L}})$, we have $w_{Z}\left(\tilde{Z}, \overline{\mathbf{L}}, \theta\left(\bar{Z}^{\prime}, \overline{\mathbf{L}}\right)\right)>w_{Z}(\tilde{Z}, \overline{\mathbf{L}}, \theta(\bar{Z}, \overline{\mathbf{L}}))$ for all $\tilde{Z} \in \mathcal{Z}$ and $\overline{\mathbf{L}} \in \mathcal{L}$.

Proof. Since $w_{Z}\left(\tilde{Z}, \overline{\mathbf{L}}, \theta\left(\bar{Z}^{\prime}, \overline{\mathbf{L}}\right)\right)=\partial F\left(\tilde{Z}, \overline{\mathbf{L}}, \theta\left(\bar{Z}^{\prime}, \overline{\mathbf{L}}\right)\right) / \partial Z, \theta\left(\bar{Z}^{\prime}, \overline{\mathbf{L}}\right) \geq \theta(\bar{Z}, \overline{\mathbf{L}})$ and $\theta\left(\bar{Z}^{\prime}, \overline{\mathbf{L}}\right) \neq \theta(\bar{Z}, \overline{\mathbf{L}})$ combined with the fact that $F$ exhibits strict increasing differences in $(Z, \theta)$ establish this result.

Corollary 2 Suppose that the hypotheses in Theorem 4 hold. Suppose in addition that $\Theta$ is a convex compact subset of $\mathbb{R}^{K}$ and that $F$ is twice continuously differentiable $(Z, \theta, \mathbf{L})$ and $\partial F(\tilde{Z}, \overline{\mathbf{L}}, \theta) / \partial Z$ is strictly increasing in $\theta$ for all $\tilde{Z} \in \mathcal{Z}$. Consider any $\bar{Z}^{\prime}, \bar{Z} \in \mathcal{Z}$, such that $\bar{Z}^{\prime}>\bar{Z}$ and $\overline{\mathbf{L}} \in \mathcal{L}$, and suppose that $\theta\left(\bar{Z}^{\prime}, \overline{\mathbf{L}}\right)$ and $\theta(\bar{Z}, \overline{\mathbf{L}})$ are in the interior of $\Theta$. Then, we have that $\theta\left(\bar{Z}^{\prime}, \overline{\mathbf{L}}\right)>\theta(\bar{Z}, \overline{\mathbf{L}})$ and $w_{Z}\left(\tilde{Z}, \overline{\mathbf{L}}, \theta\left(\bar{Z}^{\prime}, \overline{\mathbf{L}}\right)\right)>w_{Z}(\tilde{Z}, \overline{\mathbf{L}}, \theta(\bar{Z}, \overline{\mathbf{L}}))$.

Proof. This corollary follows immediately from the Strong Monotonicity Theorem, Theorem 11, in Appendix B.

An important implication of Theorem 4 and the subsequent corollaries is that we now have a global version of Theorem 3, but at the expense of introducing more structure. In particular, in addition to the relatively weak assumptions (in this context) that $\mathcal{Z}$ and $\Theta$ are lattices, we need $F$ to be (quasi-)supermodular in $\theta$ and to exhibit (strict) increasing differences in $(Z, \theta)$.

More importantly, there are limits to how much Theorems 3 and 4 can be generalized. First, Theorem 3 does not apply for large changes in $Z$. In fact, quite interestingly, we cannot take a series of small changes and turn them into a biased response for a large change in $Z$ (without the additional supermodularity and increasing differences assumption). Second, the requirement of (strict) increasing differences in $(Z, \theta)$ in Theorem 4 cannot be dispensed with, nor could it be replaced by the weaker conditions of single-crossing or quasi-supermodularity in $(Z, \theta)$ of Milgrom and Shannon (1994) - see Appendix B for definitions. Third, the assumption that $F$ should exhibit increasing differences on the convex hull of $\mathcal{Z}$ rather than $\mathcal{Z}$ itself cannot 
be dispensed with either. The following example illustrates all these features by constructing a simple economy which satisfies the local theorem, Theorem 3, at all points, but fails to yield a global version of (absolute) bias because the production function does not exhibit increasing differences in $(Z, \theta)$ on $\overline{\mathcal{Z}} \times \Theta$ (though it satisfies single crossing in $(Z, \theta)$ and in $(\theta, Z)$, and quasi-supermodularity in $(Z, \theta)$ and exhibits increasing differences on $\mathcal{Z} \times \Theta$ where $\mathcal{Z}$ is nonconvex).

Example 4 (No Global Bias Without Increasing Differences) Suppose that $F(Z, \mathbf{L}, \theta)=$ $Z+\left(Z-Z^{2} / 8\right) \theta+A(\theta)+B(\mathbf{L})$ and $Z \in \mathcal{Z}=[0,6]$ and $\Theta=[0,2]$ so that $F$ is everywhere increasing in $Z$ (with the cost of creating technologies, $C(\theta)$, incorporated into the function $A(\theta)$ ). Suppose also that $A(\theta)$ is a strictly concave and continuously differentiable real-valued function with the following boundary conditions to ensure interior solutions to the choice of technology: $A^{\prime}(0)>0$ and $A^{\prime}(2)=-\infty$ (where $A^{\prime}$ denotes $A^{\prime}$ 's derivative), and $B(\mathbf{L})$ is an increasing function. $F(Z, \mathbf{L}, \theta)$ satisfies all the conditions of Theorem 3 at all points $Z \in \mathcal{Z}=[0,6]$ (since $F$ is strictly concave in $\theta$ everywhere on $\mathcal{Z} \times \Theta=[0,6] \times[0,2]$ ), so we have local absolute equilibrium bias at all points in $\mathcal{Z} \times \Theta$.

However, $F(Z, \mathbf{L}, \theta)$ is not supermodular nor does it satisfy increasing differences in $(Z, \theta)$, since the cross-partial between $Z$ and $\theta$ changes sign depending on whether $Z$ is greater than or less than 4. Consequently, it can be verified that there will not be global equilibrium bias in this example.

To illustrate this, consider $\bar{Z}=1$ and $\bar{Z}^{\prime}=5$ as two potential supply levels of factor $Z$ and some $\overline{\mathbf{L}} \in \mathcal{L}$. It can be verified easily that $F(1, \overline{\mathbf{L}}, \theta)=1+7 \theta / 8+A(\theta)+B(\overline{\mathbf{L}})$, so that $\theta(1)$ satisfies $A^{\prime}(\theta(1))=-7 / 8$, whereas $F(5, \overline{\mathbf{L}}, \theta)=5+15 \theta / 8+A(\theta)+B(\overline{\mathbf{L}})$ so that $\theta(5)$ satisfies $A^{\prime}(\theta(5))=-15 / 8$. The strict concavity of $A(\theta)$ implies that $\theta(5)>\theta(1)$. Moreover, $w_{Z}(Z, \theta)=1+(1-Z / 4) \theta$, so $w_{Z}(5, \theta(5))=1-\theta(5) / 4<w_{Z}(5, \theta(1))=1-\theta(1) / 4$, contrary to the claim in Theorem 4.

So why does the global theorem not work, while the local theorem does? The answer is that the local theorem, Theorem 3, only refers to changes in technology that are absolutely biased at the corresponding factor proportions. Consequently, when we change $\bar{Z}$ locally, say from 1 to 1.1, this increases $\theta$, which is absolutely biased towards $Z$ around 1 . But this change is biased against $Z$ when we look at $\bar{Z}=5$. This is the fundamental reason why applying the local theorem, Theorem 3, successively will not give a global theorem and we need the additional increasing differences (supermodularity) conditions (see also footnote 33 below for further discussion on this point). 
This example can also be used to illustrate that increasing differences cannot be replaced by the weaker single-crossing property, since $F(Z, \mathbf{L}, \theta)$ may satisfy single crossing both in $(Z, \theta)$ and $(\theta, Z)$. To illustrate this, let us take $\Theta=\{\theta(1), \theta(5)\}$ and suppose that $\theta(1)=0$ and $\theta(5)=1$. Let us continue to take $\mathcal{Z}=[0,6]$. First to check single crossing in $(Z, \theta)$, note that since $\theta(1)=0, F\left(\bar{Z}^{\prime}, \overline{\mathbf{L}}, \theta(1)\right)>F(\bar{Z}, \overline{\mathbf{L}}, \theta(1))$ whenever $\bar{Z}^{\prime}>\bar{Z}$. Therefore, we only have to check that $F\left(\bar{Z}^{\prime}, \overline{\mathbf{L}}, \theta(5)\right)>F(\bar{Z}, \overline{\mathbf{L}}, \theta(5))$ whenever $\bar{Z}^{\prime}>\bar{Z}$. This immediately follows from the fact that $\theta(5)=1$, so that for all $\bar{Z}^{\prime}, \bar{Z} \in \mathcal{Z}=[0,6]$ and $\bar{Z}^{\prime}>\bar{Z}, \bar{Z}^{\prime}+\left(\bar{Z}^{\prime}-\left(\bar{Z}^{\prime}\right)^{2} / 8\right)>$ $\bar{Z}+\left(\bar{Z}-\bar{Z}^{2} / 8\right)$. To establish single crossing in $(\theta, Z)$, let us take $\theta(1)=0$ and $\theta(5)=1$ and also suppose that $A(1) \geq A(0)$. In that case, single crossing in $(\theta, Z)$ requires that whenever $\bar{Z}^{\prime}, \bar{Z} \in \mathcal{Z}=[0,6]$ and $\bar{Z}^{\prime}>\bar{Z}$, and

$$
\bar{Z}+\left(\bar{Z}-\bar{Z}^{2} / 8\right)+A(1)+B(\overline{\mathbf{L}})>\bar{Z}+A(0)+B(\overline{\mathbf{L}})
$$

it must also be the case that

$$
\bar{Z}^{\prime}+\left(\bar{Z}^{\prime}-\left(\bar{Z}^{\prime}\right)^{2} / 8\right)+A(1)+B(\overline{\mathbf{L}})>\bar{Z}^{\prime}+A(0)+B(\overline{\mathbf{L}}) .
$$

It is straightforward to verify that the first inequality will hold for all $\bar{Z} \in(0,6]$ since, in this case, $\left(\bar{Z}-\bar{Z}^{2} / 8\right)>0 \geq A(0)-A(1)$. This implies that for all $\bar{Z}^{\prime}, \bar{Z} \in \mathcal{Z}=[0,6]$ and $\bar{Z}^{\prime}>\bar{Z}$, we have $\left(\bar{Z}^{\prime}-\left(\bar{Z}^{\prime}\right)^{2} / 8\right)>0 \geq A(0)-A(1)$, establishing single crossing in $(\theta, Z)$. Since by Lemma 3 in Appendix $\mathrm{B}$, when $\mathcal{Z}$ and $\Theta$ are chains, single crossing in $(Z, \theta)$ and $(\theta, Z)$ implies quasi-supermodularity in $(Z, \theta)$, this result also implies that increasing differences in $(Z, \theta)$ cannot be replaced with quasi-supermodularity in $(Z, \theta)$.

Finally, this example also shows that the assumption that the function needs to exhibit increasing differences in $(Z, \theta)$ on the convex hull of $\mathcal{Z}, \overline{\mathcal{Z}}$, cannot be dispensed with. In particular, if we take $\mathcal{Z}=\{1,5\}$ and $\Theta=\{\theta(1), \theta(5)\}$, it can be verified that the function $F$ here satisfies supermodularity on $\mathcal{Z} \times \Theta$, and hence exhibits increasing differences in $(Z, \theta)$ on $\mathcal{Z} \times \Theta$ (see Lemma 2 in Appendix B). However, it fails to satisfy supermodularity and increasing differences on $\overline{\mathcal{Z}} \times \Theta$, where $\overline{\mathcal{Z}}=[1,5]{ }^{30}$

\footnotetext{
${ }^{30}$ To see why it is necessary for $F$ to be supermodular or exhibit increasing differences in $(Z, \theta)$ over the convex hull of $\mathcal{Z}$, note that the supermodularity of $F$ implies that for $Z^{\prime \prime}>Z^{\prime}$ and $\theta^{\prime \prime}>\theta^{\prime}$, we have

$$
F\left(Z^{\prime \prime}, \mathbf{L}, \theta^{\prime \prime}\right)+F\left(Z^{\prime}, \mathbf{L}, \theta^{\prime}\right) \geq F\left(Z^{\prime \prime}, \mathbf{L}, \theta^{\prime}\right)+F\left(Z^{\prime}, \mathbf{L}, \theta^{\prime \prime}\right) .
$$

Now, assuming differentiability and applying the Fundamental Theorem of Calculus (e.g., Rudin, 1964, Theorem 6.16) twice and using the definition of $w_{Z}$, we have

$$
\int_{Z^{\prime}}^{Z^{\prime \prime}} \int_{\theta^{\prime}}^{\theta^{\prime \prime}} \frac{\partial w_{Z}(Z, \mathbf{L}, \theta)}{\partial \theta} d \theta d Z \geq 0 .
$$
}


There is a natural and important generalization of Theorem 4 in which the supplies of a set of factors change simultaneously, and the induced change in technology is biased towards all of these factors. This is presented in the next theorem. Let the production function be $F(\mathbf{Z}, \mathbf{L}, \theta)$, where $\mathbf{Z}=\left(Z_{1}, \ldots, Z_{N}\right)$. Define the marginal products in the usual way as

$$
w_{Z j}=\frac{\partial F(\mathbf{Z}, \mathbf{L}, \theta)}{\partial Z_{j}} \text { for } j=1, \ldots, N
$$

The notion of equilibrium bias generalizes naturally.

Definition 12 Let $\overline{\mathbf{Z}} \in \mathcal{Z} \subset \mathbb{R}_{+}^{N}, \overline{\mathbf{L}} \in \mathcal{L}$ and $\theta(\overline{\mathbf{Z}}, \overline{\mathbf{L}})$ be the equilibrium technology choice in an economy with factor supplies $(\overline{\mathbf{Z}}, \overline{\mathbf{L}})$. We say that there is global absolute equilibrium bias if for any $\overline{\mathbf{Z}}^{\prime}, \overline{\mathbf{Z}} \in \mathcal{Z}, \overline{\mathbf{Z}}^{\prime} \geq \overline{\mathbf{Z}}$ implies

$$
w_{Z j}\left(\tilde{\mathbf{Z}}, \overline{\mathbf{L}}, \theta\left(\overline{\mathbf{Z}}^{\prime}, \overline{\mathbf{L}}\right)\right) \geq w_{Z j}(\tilde{\mathbf{Z}}, \overline{\mathbf{L}}, \theta(\overline{\mathbf{Z}}, \overline{\mathbf{L}})) \text { for all }(\tilde{\mathbf{Z}}, \overline{\mathbf{L}}) \in \mathcal{Z} \times \mathcal{L} \text { and for all } j=1, \ldots, N \text {. }
$$

Once again, this definition can be strengthened by introducing strict inequalities.

Theorem 5 (Generalized Global Equilibrium Bias) Consider Economy D, C or M. Suppose that $\mathcal{Z}$ and $\Theta$ are lattices, let $\overline{\mathcal{Z}}$ be the convex hull of $\mathcal{Z}$, let $\theta(\overline{\mathbf{Z}}, \overline{\mathbf{L}})$ be the equilibrium technology at factor proportions $(\overline{\mathbf{Z}}, \overline{\mathbf{L}})$, and suppose that $F(\mathbf{Z}, \mathbf{L}, \theta)$ is continuously differentiable in $\mathbf{Z}$, supermodular in $\theta$ on $\Theta$ for all $\mathbf{Z} \in \overline{\mathcal{Z}}$ and $\mathbf{L} \in \mathcal{L}$, and exhibits strictly increasing differences in $(\mathbf{Z}, \theta)$ on $\overline{\mathcal{Z}} \times \Theta$ for all $\mathbf{L} \in \mathcal{L}$, then there is global absolute equilibrium bias, i.e., for any $\overline{\mathbf{Z}}^{\prime}, \overline{\mathbf{Z}} \in \mathcal{Z}, \overline{\mathbf{Z}}^{\prime} \geq \overline{\mathbf{Z}}$ implies

$$
\theta\left(\overline{\mathbf{Z}}^{\prime}, \overline{\mathbf{L}}\right) \geq \theta(\overline{\mathbf{Z}}, \overline{\mathbf{L}}) \text { for all } \overline{\mathbf{L}} \in \mathcal{L}
$$

and

$$
w_{Z j}\left(\tilde{\mathbf{Z}}, \overline{\mathbf{L}}, \theta\left(\overline{\mathbf{Z}}^{\prime}, \overline{\mathbf{L}}\right)\right) \geq w_{Z j}(\tilde{\mathbf{Z}}, \overline{\mathbf{L}}, \theta(\overline{\mathbf{Z}}, \overline{\mathbf{L}})) \text { for all }(\tilde{\mathbf{Z}}, \overline{\mathbf{L}}) \in \mathcal{Z} \times \mathcal{L} \text { and for all } j=1, \ldots, N
$$

Proof. The proof is analogous to that of Theorem 4 and follows from Theorem 10 in Appendix B given the supermodularity of $F(\mathbf{Z}, \mathbf{L}, \theta)$ in $\theta$ and strict increasing differences in $(\mathbf{Z}, \theta)$ on $\overline{\mathcal{Z}} \times \Theta$.

It is clear that corollaries to this theorem similar to those to Theorem 4 can be stated with slightly stronger conditions. I omit these to avoid repetition. Also, as in Theorem 4, However, this does not guarantee that

$$
\int_{\theta^{\prime}}^{\theta^{\prime \prime}} \frac{\partial w_{Z}(Z, \mathbf{L}, \theta)}{\partial \theta} d \theta \geq 0
$$

for all $Z \in\left[Z^{\prime}, Z^{\prime \prime}\right]$ unless $F$ is supermodular over the convex hull of $\left\{Z^{\prime}, Z^{\prime \prime}\right\}$. 
supermodularity in $\theta$ can be weakened to quasi-supermodularity in $\theta$, or strict increasing differences can be relaxed to increasing differences and the comparison of $\theta\left(\overline{\mathbf{Z}}^{\prime}, \overline{\mathbf{L}}\right)$ to $\theta(\overline{\mathbf{Z}}, \overline{\mathbf{L}})$ would apply for the greatest and the least elements of the equilibrium technology set.

\section{$5 \quad$ Strong Absolute Equilibrium Bias}

The results in Section 4 concern "weak" bias in the sense that they compare marginal products at a given level of factor supplies (in response to a change in $\theta$ induced by a change in $Z$ ). This section provides the conditions under which equilibrium bias will be "strong" in the sense that once technology has adjusted, the increase in the supply of factor $Z$ will increase its marginal product (price). As noted in the Introduction, this is particularly important because it emphasizes the central role of the equilibrium structure in the analysis here, since such a result would not be possible in the neoclassical production theory.

Example 1 above illustrated the possibility of strong relative bias where technology might be so responsive to factor supply changes that when a factor becomes more abundant, its relative price and marginal product increase rather than decrease. Although somewhat counterintuitive at first, this is also a possibility in the class of models studied here. But we will see that it requires some type of nonconvexity either in the technology set, $\Theta$, or in the production possibilities set by allowing for a structure similar to that of Economy C or Economy M. First, I define strong absolute bias, and to simplify the discussion, from now on, I focus on changes in a single factor. Recall throughout that equilibrium technology is still a solution to the maximization problem in (34).

Definition 13 Suppose that $N=1$. Let $\theta(Z, \mathbf{L}) \in \Theta$ be the equilibrium technology choice in an economy with factor proportions $(Z, \mathbf{L})$. We say that there is strong absolute equilibrium bias at $\left(\left\{\bar{Z}, \bar{Z}^{\prime}\right\}, \overline{\mathbf{L}}\right)$ if for some $\overline{\mathbf{L}} \in \mathcal{L}$ and $\bar{Z}, \bar{Z}^{\prime} \in \mathcal{Z}$ with $\bar{Z}^{\prime}>\bar{Z}$, we have

$$
w_{Z}\left(\bar{Z}^{\prime}, \overline{\mathbf{L}}, \theta\left(\bar{Z}^{\prime}, \overline{\mathbf{L}}\right)\right)>w_{Z}(\bar{Z}, \overline{\mathbf{L}}, \theta(\bar{Z}, \overline{\mathbf{L}})) .
$$

Similarly, suppose that $\Theta \subset \mathbb{R}^{K}, w_{Z}(\bar{Z}, \overline{\mathbf{L}}, \theta(\bar{Z}, \overline{\mathbf{L}}))$ is differentiable in $Z$ and $\partial \theta_{j}(\bar{Z}, \overline{\mathbf{L}}) / \partial Z$ exists at $(\bar{Z}, \overline{\mathbf{L}})$ for all $j=1, \ldots, K$. Then we say that there is strong absolute equilibrium bias at $(\bar{Z}, \overline{\mathbf{L}}) \in \mathcal{Z} \times \mathcal{L}$ if

$$
\frac{d w_{Z}(\bar{Z}, \overline{\mathbf{L}}, \theta(\bar{Z}, \overline{\mathbf{L}}))}{d Z}=\frac{\partial w_{Z}(\bar{Z}, \overline{\mathbf{L}}, \theta(\bar{Z}, \overline{\mathbf{L}}))}{\partial Z}+\sum_{j=1}^{K} \frac{\partial w_{Z}(\bar{Z}, \overline{\mathbf{L}}, \theta(\bar{Z}, \overline{\mathbf{L}}))}{\partial \theta_{j}} \frac{\partial \theta_{j}(\bar{Z}, \bar{L})}{\partial Z}>0
$$


Note that in this definition I use $d w_{Z}(\bar{Z}, \overline{\mathbf{L}}, \theta(\bar{Z}, \overline{\mathbf{L}})) / d Z$ to denote the total derivative, while $\partial w_{Z}(\bar{Z}, \overline{\mathbf{L}}, \theta(\bar{Z}, \overline{\mathbf{L}})) / \partial Z$ denotes the partial derivative holding $\theta=\theta(\bar{Z}, \overline{\mathbf{L}})$. The next theorem shows that there cannot be strong absolute bias in Economy $\mathrm{D}$ if $\Theta$ is a convex subset of $\mathbb{R}^{K}$.

Theorem 6 (No Strong Bias in Economy D) Suppose that $\Theta$ is a convex subset of $\mathbb{R}^{K}$, $F$ is twice continuously differentiable in $(Z, \theta)$, let the equilibrium technology at factor supplies $(\bar{Z}, \overline{\mathbf{L}})$ be $\theta(\bar{Z}, \overline{\mathbf{L}})$, and assume that $\partial \theta_{j}(\bar{Z}, \overline{\mathbf{L}}) / \partial Z$ exists at $(\bar{Z}, \overline{\mathbf{L}})$ for all $j=1, \ldots, K$. Then there cannot be strong absolute bias in Economy D.

Proof. Let us start with the local result and the case with $\theta \in \mathbb{R}$. Let factor supplies be $(\bar{Z}, \overline{\mathbf{L}})$. Strong absolute bias corresponds to

$$
\frac{d w_{Z}(\bar{Z}, \overline{\mathbf{L}}, \theta(\bar{Z}, \overline{\mathbf{L}}))}{d Z}=\frac{\partial w_{Z}(\bar{Z}, \overline{\mathbf{L}}, \theta(\bar{Z}, \overline{\mathbf{L}}))}{\partial Z}+\frac{\partial w_{Z}(\bar{Z}, \overline{\mathbf{L}}, \theta(\bar{Z}, \overline{\mathbf{L}}))}{\partial \theta} \frac{\partial \theta(\bar{Z}, \bar{L})}{\partial Z}>0 .
$$

This is equivalent to

$$
\frac{d w_{Z}(\bar{Z}, \overline{\mathbf{L}}, \theta(\bar{Z}, \overline{\mathbf{L}}))}{d Z}=\frac{\partial^{2} F(\bar{Z}, \overline{\mathbf{L}}, \theta(\bar{Z}, \overline{\mathbf{L}}))}{\partial Z^{2}}+\frac{\partial w_{Z}(\bar{Z}, \overline{\mathbf{L}}, \theta(\bar{Z}, \overline{\mathbf{L}}))}{\partial \theta} \frac{\partial \theta(\bar{Z}, \bar{L})}{\partial Z}>0 .
$$

Recall from the proof of Theorem 3 that when $\theta(\bar{Z}, \overline{\mathbf{L}})$ is in the interior of $\Theta$, the first-order condition (38) holds, and we have:

$$
\frac{\partial \theta(\bar{Z}, \overline{\mathbf{L}})}{\partial Z}=-\frac{\partial^{2} F(\bar{Z}, \overline{\mathbf{L}}, \theta(\bar{Z}, \overline{\mathbf{L}})) / \partial \theta \partial Z}{\partial^{2} F(\bar{Z}, \overline{\mathbf{L}}, \theta(\bar{Z}, \overline{\mathbf{L}})) / \partial \theta^{2}}=-\frac{\partial w_{Z}(\bar{Z}, \overline{\mathbf{L}}, \theta(\bar{Z}, \overline{\mathbf{L}})) / \partial \theta}{\partial^{2} F(\bar{Z}, \overline{\mathbf{L}}, \theta(\bar{Z}, \overline{\mathbf{L}})) / \partial \theta^{2}} .
$$

so strong absolute bias would imply

$$
\frac{d w_{Z}(\bar{Z}, \overline{\mathbf{L}}, \theta(\bar{Z}, \overline{\mathbf{L}}))}{d Z}=\frac{\partial^{2} F(\bar{Z}, \overline{\mathbf{L}}, \theta(\bar{Z}, \overline{\mathbf{L}}))}{\partial Z^{2}}-\frac{\left(\partial^{2} F(\bar{Z}, \overline{\mathbf{L}}, \theta(\bar{Z}, \overline{\mathbf{L}})) / \partial \theta \partial Z\right)^{2}}{\partial^{2} F(\bar{Z}, \overline{\mathbf{L}}, \theta(\bar{Z}, \overline{\mathbf{L}})) / \partial \theta^{2}}>0 .
$$

To see that this is impossible, first note that since $\partial \theta(\bar{Z}, \bar{L}) / \partial Z$ exists, $\partial^{2} F(\bar{Z}, \overline{\mathbf{L}}, \theta(\bar{Z}, \overline{\mathbf{L}})) / \partial \theta^{2}<$ 0 (from the non-singularity, $\partial^{2} F(\bar{Z}, \overline{\mathbf{L}}, \theta) / \partial \theta_{j}^{2} \neq 0$ combined with the fact that $\theta(\bar{Z}, \bar{L})$ is a solution to (34), so that $\left.\partial^{2} F(\bar{Z}, \overline{\mathbf{L}}, \theta(\bar{Z}, \overline{\mathbf{L}})) / \partial \theta^{2} \leq 0\right)$; and second that the joint concavity of $F(\bar{Z}, \overline{\mathbf{L}}, \theta(\bar{Z}, \overline{\mathbf{L}}))$ in $(Z, \theta)$ implies that the Hessian of $F$ in $(Z, \theta), \nabla_{(Z, \theta)(Z, \theta)}^{2} F$, is negative semi-definite, thus every principle minor of $\nabla_{(Z, \theta)(Z, \theta)}^{2} F$ of even order has to be nonnegative (see, e.g., Simon and Blume, 1994, Theorem 16.2). This implies

$$
\partial^{2} F(\bar{Z}, \overline{\mathbf{L}}, \theta(\bar{Z}, \overline{\mathbf{L}})) / \partial \theta^{2} \times\left(\partial^{2} F(\bar{Z}, \overline{\mathbf{L}}, \theta(\bar{Z}, \overline{\mathbf{L}})) / \partial Z^{2}\right) \geq\left(\partial^{2} F(\bar{Z}, \overline{\mathbf{L}}, \theta(\bar{Z}, \overline{\mathbf{L}})) / \partial \theta \partial Z\right)^{2},
$$

which combined with $\partial^{2} F(\bar{Z}, \overline{\mathbf{L}}, \theta(\bar{Z}, \overline{\mathbf{L}})) / \partial \theta^{2}<0$ contradicts (42), proving the claim for the case of $\theta \in \mathbb{R}$ and in the interior of $\Theta$. When $\theta$ is at the boundary of $\Theta$, either (38) 
holds, in which case the same argument applies (since by hypothesis $\partial \theta(\bar{Z}, \bar{L}) / \partial Z$ exists even at this point). Alternatively, $\partial F(\bar{Z}, \overline{\mathbf{L}}, \theta(\bar{Z}, \overline{\mathbf{L}})) / \partial \theta<0$. However, in this case since $F$ is twice continuously differentiable in $(Z, \theta)$ and $\partial \theta(\bar{Z}, \bar{L}) / \partial Z$ exists, a sufficiently small change in $Z$ will leave $\partial F(\bar{Z}, \overline{\mathbf{L}}, \theta(\bar{Z}, \overline{\mathbf{L}})) / \partial \theta<0$ and thus $\partial \theta(\bar{Z}, \overline{\mathbf{L}}) / \partial Z=0$. Consequently, $d w_{Z}(\bar{Z}, \overline{\mathbf{L}}, \theta(\bar{Z}, \overline{\mathbf{L}})) / d Z=\partial w_{Z}(\bar{Z}, \overline{\mathbf{L}}, \theta(\bar{Z}, \overline{\mathbf{L}})) / \partial Z \leq 0$.

Next, to prove this result with multiple dimensions of technology, i.e., with $\theta \in \mathbb{R}^{K}$ for $K>1$, note that when $\theta(\bar{Z}, \overline{\mathbf{L}})$ is in the interior of $\Theta$, we have

$$
\begin{aligned}
\frac{d w_{Z}(\bar{Z}, \overline{\mathbf{L}}, \theta(\bar{Z}, \overline{\mathbf{L}}))}{d Z} & =\frac{\partial^{2} F(\bar{Z}, \overline{\mathbf{L}}, \theta(\bar{Z}, \overline{\mathbf{L}}))}{\partial Z^{2}} \\
& -\left[\nabla_{\theta Z}^{2} F(\bar{Z}, \overline{\mathbf{L}}, \theta(\bar{Z}, \overline{\mathbf{L}}))\right]^{\prime}\left[\nabla_{\theta \theta}^{2} F(\bar{Z}, \overline{\mathbf{L}}, \theta(\bar{Z}, \overline{\mathbf{L}}))\right]^{-1}\left[\nabla_{\theta Z}^{2} F(\bar{Z}, \overline{\mathbf{L}}, \theta(\bar{Z}, \overline{\mathbf{L}}))\right] .
\end{aligned}
$$

Since $\theta(\bar{Z}, \overline{\mathbf{L}})$ is a solution to $(34), \nabla_{\theta \theta}^{2} F(\bar{Z}, \overline{\mathbf{L}}, \theta(\bar{Z}, \overline{\mathbf{L}}))$ is negative semi-definite and symmetric (since it is a Hessian). Moreover, since $\nabla_{Z} \theta(\bar{Z}, \overline{\mathbf{L}})$ exists by hypothesis, $\nabla_{\theta \theta}^{2} F(\bar{Z}, \overline{\mathbf{L}}, \theta(\bar{Z}, \overline{\mathbf{L}}))$ is non-singular, so it is negative definite and symmetric. This implies that its inverse $\left[\nabla_{\theta \theta}^{2} F(\bar{Z}, \overline{\mathbf{L}}, \theta(\bar{Z}, \overline{\mathbf{L}}))\right]^{-1}$ is also negative definite and symmetric, and moreover $\partial^{2} F(\bar{Z}, \overline{\mathbf{L}}, \theta) / \partial Z^{2} \leq 0$ by the concavity of $F$ in $Z$ (from Assumption 1, 1', 2 or 2').

Lemma 4 in Appendix $\mathrm{B}$ shows that an $n \times n$ matrix

$$
B=\left(\begin{array}{cc}
C & v \\
v^{\prime} & b
\end{array}\right)
$$

where $C$ is an $(n-1) \times(n-1)$ symmetric negative definite, $b$ is a scalar, and $v$ is an $(n-1) \times 1$ column vector, is negative semi-definite if and only if $b-v^{\prime} C^{-1} v \leq 0$ where $C^{-1}$ is the inverse of $C$. Let us now apply this lemma with $b=\partial^{2} F(\bar{Z}, \overline{\mathbf{L}}, \theta) / \partial Z^{2} \leq 0, C=\left[\nabla_{\theta \theta}^{2} F(\bar{Z}, \overline{\mathbf{L}}, \theta(\bar{Z}, \overline{\mathbf{L}}))\right]$, and $v=\left[\nabla_{\theta Z}^{2} F(\bar{Z}, \overline{\mathbf{L}}, \theta(\bar{Z}, \overline{\mathbf{L}}))\right]$, which implies that the expression in (43) is equal to $b-$ $v^{\prime} C^{-1} v$. The fact that $F$ is jointly concave in $(Z, \theta)$ implies that the Hessian of $F$ with respect to $(Z, \theta), \nabla_{(Z, \theta)(Z, \theta)}^{2} F$ is negative semi-definite. Therefore, from Lemma $4, b-v^{\prime} C^{-1} v \leq 0$ and (43) cannot be positive, completing the proof of the local result. The proof for the case where $\theta(\bar{Z}, \overline{\mathbf{L}})$ is at the boundary of $\Theta$ is analogous to the one above for $\Theta \subset \mathbb{R}$.

Finally, to prove the global result, i.e., that strong bias is impossible in this economy for any change in factor supplies, note that from the Fundamental Theorem of Calculus, for any $\bar{Z}^{\prime}>\bar{Z}$, we have

$$
w_{Z}\left(\bar{Z}^{\prime}, \overline{\mathbf{L}}, \theta\left(\bar{Z}^{\prime}, \overline{\mathbf{L}}\right)\right)-w_{Z}(\bar{Z}, \overline{\mathbf{L}}, \theta(\bar{Z}, \overline{\mathbf{L}}))=\int_{\tilde{Z}}^{\bar{Z}^{\prime}} \frac{d w_{Z}(Z, \overline{\mathbf{L}}, \theta(Z, \overline{\mathbf{L}}))}{d Z} d Z .
$$

Since $d w_{Z}(Z, \overline{\mathbf{L}}, \theta(\bar{Z}, \overline{\mathbf{L}})) / d Z \leq 0$ for all $Z \in\left[\bar{Z}, \bar{Z}^{\prime}\right]$, the integral is nonpositive, establishing the global result. 
The result in this theorem is not surprising. In Economy D, the production possibilities set is convex, so the marginal product of each factor is decreasing in its supply even after technology adjusts. In contrast, once we allow for nonconvexities (and factor demands and technology to be chosen by different agents), the results are very different. To illustrate the importance of nonconvexities, I now look at Economy D with a nonconvex technology set $\Theta,{ }^{31}$ and at Economies $\mathrm{C}$ or $\mathrm{M}$, which allow for natural nonconvexities. I establish that in both cases strong absolute bias is possible.

Theorem 7 (Strong Absolute Bias) Strong absolute equilibrium bias is possible either in Economy D with a nonconvex technology set, $\Theta$, or in Economy $\mathrm{C}$ or $\mathrm{M}$.

This theorem will be proved by providing two examples with strong absolute equilibrium bias.

Example 5 (Strong Absolute Bias in Economy D) Take Economy D and suppose that $F(Z, \mathbf{L}, \theta)=Z^{1 / 2} \theta^{1 / 2}-\theta+B(\mathbf{L})$ and $\Theta=\{1,4\}$. Imagine an increase in $\bar{Z}$ from 4 to $9+\varepsilon$ where $\varepsilon>0$. It is straightforward to check that for any $\overline{\mathbf{L}} \in \mathcal{L}, F(4, \overline{\mathbf{L}}, 1)=2-1+B(\overline{\mathbf{L}})>$ $F(4, \overline{\mathbf{L}}, 4)=4-4+B(\overline{\mathbf{L}})$, so $\theta(4)=1$. In contrast, $F(9+\varepsilon, \overline{\mathbf{L}}, 4)=(9+\varepsilon)^{1 / 2} 2-4+B(\overline{\mathbf{L}})>$ $F(9+\varepsilon, \overline{\mathbf{L}}, 1)=(9+\varepsilon)^{1 / 2}-1+B(\overline{\mathbf{L}})$, so that $\theta(9+\varepsilon)=4$ (in particular, the two sides are equal when $\varepsilon=0$, and the left-hand side increases faster in $\varepsilon$ ). Therefore, an increase in $\bar{Z}$ from 4 to $9+\varepsilon$ will induce a change in technology from $\theta(4)=1$ to $\theta(9+\varepsilon)=4$. The price (marginal product) of factor $Z$ is given by $w_{Z}(\bar{Z}, \overline{\mathbf{L}}, \theta)=(\theta / \bar{Z})^{1 / 2} / 2$, so the change in this price resulting from the increase in $\bar{Z}$ (after technology adjusts) is $w_{Z}(\bar{Z}=9+\varepsilon, \overline{\mathbf{L}}, 4)-$ $w_{Z}(\bar{Z}=4, \overline{\mathbf{L}}, 1)=(4 /(9+\varepsilon))^{1 / 2} / 2-(1 / 4)^{1 / 2} / 2 \simeq 1 / 3-1 / 4>0$ for $\varepsilon$ sufficiently small, establishing the possibility of strong absolute bias in Economy D with a nonconvex technology set.

Example 6 (Strong Absolute Bias in Economy $\boldsymbol{C}$ or $\boldsymbol{M}$ ) Next, consider Economy C or $\mathrm{M}$, and to illustrate that a nonconvex technology set is not necessary in these economies, take $\Theta=\mathbb{R}$. Suppose $F(Z, \mathbf{L}, \theta)=4 Z^{1 / 2}+Z \theta-\theta^{2} / 2+B(\mathbf{L})$ (again with the cost of creating new technologies, $C(\theta)$, incorporated into this function). Clearly $F$ is not jointly concave in $Z$ and $\theta$ (for $Z>1$ ) but is strictly concave in $Z$ and $\theta$ individually. As Theorem 8 below will show, this is a crucial feature in generating strong (absolute) bias. Now consider a change from $\bar{Z}=1$

\footnotetext{
${ }^{31}$ In Economy D, when Assumption 1 applies the technology set $\Theta$ is also assumed to be convex. This assumption can be relaxed. Recall also that convexity of $\Theta$ is not required by Assumption 1', which only requires $\mathcal{L}$ and $\mathcal{Z}$ to be convex.
} 
to $\bar{Z}=4$. Clearly, the first-order necessary and sufficient condition for technology choice gives $\theta(\bar{Z}, \overline{\mathbf{L}})=\theta(\bar{Z})=\bar{Z}$. Therefore, $\theta(\bar{Z}=1)=1$ while $\theta(\bar{Z}=4)=4$. Moreover, for any $\overline{\mathbf{L}} \in \mathcal{L}$, $w_{Z}(\bar{Z}, \overline{\mathbf{L}}, \theta)=2 Z^{-1 / 2}+\theta$. Therefore, $w_{Z}(\bar{Z}=1, \overline{\mathbf{L}}, \theta(1))=3<w_{Z}(\bar{Z}=4, \overline{\mathbf{L}}, \theta(4))=5$, establishing strong (absolute) equilibrium bias between $\bar{Z}=1$ to $\bar{Z}=4$. In fact, Theorem 8 implies that there will be local strong equilibrium bias in this example for all $\bar{Z} \geq 1$, and Theorem 9 then implies that there will be global strong equilibrium bias between any $\bar{Z}^{\prime}$ and $\bar{Z}$ with $\bar{Z}^{\prime}>\bar{Z} \geq 1$.

The importance of Theorem 7 is that, contrary to the predictions of the standard production theory, where the increase in the supply of a factor always reduces its price (and marginal product), with endogenous technology choice or technological change, the price of a factor which has become more abundant can increase. Examples 5 and 6 show that it is straightforward to construct economies in which there is such strong bias.

This theorem also distinguishes the approach in this paper from the literature on the LeChatelier principle, which looks at the decision problem of a single firm. As is well-known, the firm's demand curve for a factor is always downward sloping in its own price (e.g., Mas-Colell, Whinston and Green, 1995, Proposition 5.C.2), so the equilibrium structure (in particular, the equilibrium with aggregate nonconvexities) is important for the results in this paper, especially for the possibility of strong equilibrium bias.

The fact that strong equilibrium bias is possible in Economy D when the technology set $\Theta$ is nonconvex is also interesting. Although many existing approaches to technology, such as the models of endogenous technological progress (e.g., Romer, 1990, Grossman and Helpman, 1991, Aghion and Howitt, 1992), view technology as a scalar in a convex set, as already discussed in the Introduction, for many important technological choices, switching between discrete technologies may be quite important. If this is the case, allowing $\Theta$ to be nonconvex is important and realistic (recall that Theorems 4 and 5 above do not require $\Theta$ to be convex).

Finally, as stated in the Introduction and already hinted in the discussion, "greater nonconvexity" makes it more likely that the economy will feature strong absolute bias. This is formalized in the next theorem. Recall that in Economy $\mathrm{C}$ or $\mathrm{M}, F(Z, \mathbf{L}, \theta)=G(Z, \mathbf{L}, \theta)-C(\theta)$, so marginal product of $Z$ is equivalently given by the derivative of function $F$ or $G$. Recall also that $F(Z, \mathbf{L}, \theta)$ is always concave in $(Z, \mathbf{L})$ (from Assumption 1, 1', 2 or 2') and has to be locally concave in $\theta$ for $\theta(\bar{Z}, \overline{\mathbf{L}})$ to be an equilibrium technology (i.e., a solution to the maximization problem in (34)). Recall that if $F$ is jointly concave in $(Z, \theta)$ at $(Z, \theta(\bar{Z}, \overline{\mathbf{L}}))$, its Hessian with respect to $(Z, \theta), \nabla^{2} F_{(Z, \theta)(Z, \theta)}$, is negative semi-definite at this point (though 
negative semi-definiteness is not sufficient for local joint concavity).

Theorem 8 (Nonconvexity and Strong Bias) Consider Economy C or M. Suppose that $\Theta$ is a convex subset of $\mathbb{R}^{K}, F$ is twice continuously differentiable in $(Z, \theta)$, let $\theta(\bar{Z}, \overline{\mathbf{L}})$ be the equilibrium technology at factor supplies $(\bar{Z}, \overline{\mathbf{L}})$ and assume that $\theta(\bar{Z}, \overline{\mathbf{L}})$ is in the interior of $\Theta$ and that $\partial \theta_{j}(\bar{Z}, \overline{\mathbf{L}}) / \partial Z$ exists at $(\bar{Z}, \overline{\mathbf{L}})$ for all $j=1, \ldots, K$. Then there is strong absolute bias at $(\bar{Z}, \overline{\mathbf{L}})$ if and only if $F(Z, \mathbf{L}, \theta)$ 's Hessian in $(Z, \theta), \nabla^{2} F_{(Z, \theta)(Z, \theta)}$, is not negative semidefinite at $(\bar{Z}, \overline{\mathbf{L}}, \theta(\bar{Z}, \overline{\mathbf{L}}))$.

Proof. Let us start with the case where $\Theta \subset \mathbb{R}$. Since, by hypothesis, $\theta$ is in the interior of $\Theta$, the first-order condition, equation (38) from the proof of Theorem 3, holds. Then recall the proof of Theorem 6 and in particular, equation (42), where it was established that for the case of $\theta \in \mathbb{R}$ :

$$
\frac{d w_{Z}(\bar{Z}, \overline{\mathbf{L}}, \theta(\bar{Z}, \overline{\mathbf{L}}))}{d Z}=\frac{\partial^{2} F(\bar{Z}, \overline{\mathbf{L}}, \theta(\bar{Z}, \overline{\mathbf{L}}))}{\partial Z^{2}}-\frac{\left(\partial^{2} F(\bar{Z}, \overline{\mathbf{L}}, \theta(\bar{Z}, \overline{\mathbf{L}})) / \partial \theta \partial Z\right)^{2}}{\partial^{2} F(\bar{Z}, \overline{\mathbf{L}}, \theta(\bar{Z}, \overline{\mathbf{L}})) / \partial \theta^{2}} .
$$

Again from Assumption 1, 1', 2 or 2', $F$ is concave in $Z$, so $\partial^{2} F(\bar{Z}, \overline{\mathbf{L}}, \theta) / \partial Z^{2} \leq 0$, and from the fact that $\theta(\bar{Z}, \overline{\mathbf{L}})$ is a solution to $(34)$ and $\partial \theta(\bar{Z}, \overline{\mathbf{L}}) / \partial Z$ exists, we also have $\partial^{2} F(\bar{Z}, \overline{\mathbf{L}}, \theta(\bar{Z}, \overline{\mathbf{L}})) / \partial \theta^{2}<0$. Then the fact that $F(\bar{Z}, \overline{\mathbf{L}}, \theta(\bar{Z}, \overline{\mathbf{L}}))$ 's Hessian, $\nabla^{2} F_{(Z, \theta)(Z, \theta)}$, is not negative semi-definite at $(\bar{Z}, \overline{\mathbf{L}}, \theta(\bar{Z}, \overline{\mathbf{L}}))$ implies that

$$
\left(\partial^{2} F(\bar{Z}, \overline{\mathbf{L}}, \theta(\bar{Z}, \overline{\mathbf{L}})) / \partial \theta^{2}\right) \times\left(\partial^{2} F(\bar{Z}, \overline{\mathbf{L}}, \theta(\bar{Z}, \overline{\mathbf{L}})) / \partial Z^{2}\right)<\left(\partial^{2} F(\bar{Z}, \overline{\mathbf{L}}, \theta(\bar{Z}, \overline{\mathbf{L}})) / \partial \theta \partial Z\right)^{2} .
$$

Since at the optimal technology choice, $\partial^{2} F(\bar{Z}, \overline{\mathbf{L}}, \theta(\bar{Z}, \overline{\mathbf{L}})) / \partial \theta^{2}<0$, this immediately yields

$$
\frac{d w_{Z}(\bar{Z}, \overline{\mathbf{L}}, \theta(\bar{Z}, \overline{\mathbf{L}}))}{d Z}>0
$$

establishing strong absolute bias at $(\bar{Z}, \overline{\mathbf{L}}, \theta(\bar{Z}, \overline{\mathbf{L}}))$ as claimed in the theorem.

Conversely, if $\nabla^{2} F_{(Z, \theta)(Z, \theta)}$ is negative semi-definite at $(\bar{Z}, \overline{\mathbf{L}}, \theta(\bar{Z}, \overline{\mathbf{L}}))$, then

$$
\left(\partial^{2} F(\bar{Z}, \overline{\mathbf{L}}, \theta(\bar{Z}, \overline{\mathbf{L}})) / \partial \theta^{2}\right) \times\left(\partial^{2} F(\bar{Z}, \overline{\mathbf{L}}, \theta(\bar{Z}, \overline{\mathbf{L}})) / \partial Z^{2}\right) \geq\left(\partial^{2} F(\bar{Z}, \overline{\mathbf{L}}, \theta(\bar{Z}, \overline{\mathbf{L}})) / \partial \theta \partial Z\right)^{2},
$$

which, together with $\partial^{2} F(\bar{Z}, \overline{\mathbf{L}}, \theta(\bar{Z}, \overline{\mathbf{L}})) / \partial \theta^{2}<0$, implies that $d w_{Z}(\bar{Z}, \overline{\mathbf{L}}, \theta(\bar{Z}, \overline{\mathbf{L}})) / d Z \leq 0$, establishing that for strong bias at $(\bar{Z}, \overline{\mathbf{L}}, \theta(\bar{Z}, \overline{\mathbf{L}}))$ we need $\nabla^{2} F_{(Z, \theta)(Z, \theta)}$ not to be negative semi-definite at $(\bar{Z}, \overline{\mathbf{L}}, \theta(\bar{Z}, \overline{\mathbf{L}}))$.

Now for the general case where $\Theta \subset \mathbb{R}^{K}$ and $\theta(\bar{Z}, \overline{\mathbf{L}})$ is in the interior of $\Theta$, the overall change in the price of factor $Z$ is given by (43) in the proof of Theorem 6, i.e.,

$$
\begin{aligned}
\frac{d w_{Z}(\bar{Z}, \overline{\mathbf{L}}, \theta(\bar{Z}, \overline{\mathbf{L}}))}{d Z} & =\frac{\partial^{2} F(\bar{Z}, \overline{\mathbf{L}}, \theta(\bar{Z}, \overline{\mathbf{L}}))}{\partial Z^{2}} \\
& -\left[\nabla_{\theta Z}^{2} F(\bar{Z}, \overline{\mathbf{L}}, \theta(\bar{Z}, \overline{\mathbf{L}}))\right]^{\prime}\left[\nabla_{\theta \theta}^{2} F(\bar{Z}, \overline{\mathbf{L}}, \theta(\bar{Z}, \overline{\mathbf{L}}))\right]^{-1}\left[\nabla_{\theta Z}^{2} F(\bar{Z}, \overline{\mathbf{L}}, \theta(\bar{Z}, \overline{\mathbf{L}}))\right] .
\end{aligned}
$$


Again by the same arguments, $\partial^{2} F(\bar{Z}, \overline{\mathbf{L}}, \theta) / \partial Z^{2} \leq 0$ and $\nabla_{\theta \theta}^{2} F(\bar{Z}, \overline{\mathbf{L}}, \theta(\bar{Z}, \overline{\mathbf{L}}))$ is negative definite and symmetric (which implies that its inverse $\left[\nabla_{\theta \theta}^{2} F(\bar{Z}, \overline{\mathbf{L}}, \theta(\bar{Z}, \overline{\mathbf{L}}))\right]^{-1}$ is also negative definite and symmetric). Suppose that $\nabla^{2} F_{(Z, \theta)(Z, \theta)}$ is not negative semi-definite at $(\bar{Z}, \overline{\mathbf{L}}, \theta(\bar{Z}, \overline{\mathbf{L}}))$. Then from Lemma 4 in Appendix B and using the same notation as in the proof of Theorem 6 , let $B=\nabla^{2} F_{(Z, \theta)(Z, \theta)}, b=\partial^{2} F(\bar{Z}, \overline{\mathbf{L}}, \theta) / \partial Z^{2}, C=\left[\nabla_{\theta \theta}^{2} F(\bar{Z}, \overline{\mathbf{L}}, \theta(\bar{Z}, \overline{\mathbf{L}}))\right]$, and $v=\left[\nabla_{\theta Z}^{2} F(\bar{Z}, \overline{\mathbf{L}}, \theta(\bar{Z}, \overline{\mathbf{L}}))\right]$, so that $(44)$ is equal to $b-v^{\prime} C^{-1} v$ evaluated at $(\bar{Z}, \overline{\mathbf{L}}, \theta(\bar{Z}, \overline{\mathbf{L}}))$. Lemma 4 immediately implies that if $\nabla^{2} F_{(Z, \theta)(Z, \theta)}$ is not negative semi-definite at $(\bar{Z}, \overline{\mathbf{L}}, \theta(\bar{Z}, \overline{\mathbf{L}}))$, then $b-v^{\prime} C^{-1} v>0$, so that $d w_{Z}(\bar{Z}, \overline{\mathbf{L}}, \theta(\bar{Z}, \overline{\mathbf{L}})) / d Z>0$ and there is strong bias at $(\bar{Z}, \overline{\mathbf{L}}, \theta(\bar{Z}, \overline{\mathbf{L}}))$.

Conversely, again from Lemma 4 , if $\nabla^{2} F_{(Z, \theta)(Z, \theta)}$ is negative semi-definite at $(\bar{Z}, \overline{\mathbf{L}}, \theta(\bar{Z}, \overline{\mathbf{L}}))$, then $b-v^{\prime} C^{-1} v \leq 0$ and $d w_{Z}(\bar{Z}, \overline{\mathbf{L}}, \theta(\bar{Z}, \overline{\mathbf{L}})) / d Z \leq 0$, so that there is no strong bias at $(\bar{Z}, \overline{\mathbf{L}}, \theta(\bar{Z}, \overline{\mathbf{L}}))$, completing the proof.

This theorem therefore shows that in Economy $\mathrm{C}$ or $\mathrm{M}$ strong absolute bias will obtain if and only if the Hessian of the function $F(Z, \mathbf{L}, \theta)$ fails to be negative semi-definite, which loosely corresponds to $F$ failing to be jointly concave in $(Z, \theta)$. It therefore highlights the importance of nonconvexities in generating strong equilibrium bias of technology. ${ }^{32}$

More specifically, recall that for Economies $\mathrm{C}$ and $\mathrm{M}$, we have $Z$ and $\theta$ chosen by different agents. For example, in Economy M, final good producers choose their input demands, while the technology monopolist chooses technology. This implies that we are at the maximum of $F$ when we change only $Z$ or only $\theta$. But this does not guarantee that we are at the maximum in the entire $(Z, \theta)$ plane. In other words, the equilibrium may be a saddle point rather than a maximum of the function $F$. When this is the case, a change in $Z$ will induce $\theta$ to change in the direction of further increasing $F$, and consequently, the marginal product of factor $Z$ will increase. Contrasting this result with Theorem 6 , we see the importance of the equilibrium structure and nonconvexity. As shown in Theorem 6, in Economy D with a convex technology set $\Theta$, equilibrium ensures that we are at a maximum, so strong equilibrium bias is not possible. Strong equilibrium bias is only possible when equilibrium results from the interaction of choices by different agents (e.g., final good producers and the technology monopolist), or when the technology set $\Theta$ is itself nonconvex.

Note also that Theorem 8 not only specifies the conditions for strong equilibrium bias, but also highlights that these are not very restrictive. In fact, inspection of Example 6 shows

\footnotetext{
${ }^{32}$ The assumption that $\theta(\bar{Z}, \overline{\mathbf{L}})$ is in the interior of $\Theta$ is adopted to obtain an "if and only if" theorem. When $\theta(\bar{Z}, \overline{\mathbf{L}})$ is at the boundary of $\Theta$, strong equilibrium bias is again possible, but failure of negative semi-definiteness is no longer sufficient.
} 
that it is very straightforward to construct cases in which equilibria in Economies $\mathrm{C}$ and $\mathrm{M}$ correspond to saddle points, and thus satisfy the conditions of Theorem 8 .

Finally, it is also possible to provide a generalization of Theorem 8 for large changes in supplies (corresponding to strong bias between factor supplies $\left\{\bar{Z}, \bar{Z}^{\prime}\right\}$ as in the first part of Definition 13). In particular, we have: ${ }^{33}$

Theorem 9 (Nonconvexity and Global Strong Bias) Consider Economy C or M. Suppose that $\Theta$ is a convex subset of $\mathbb{R}^{K}, F$ is twice continuously differentiable in $(Z, \theta)$, let $\bar{Z}, \bar{Z}^{\prime} \in$ $\mathcal{Z}$, with $\bar{Z}^{\prime}>\bar{Z}, \overline{\mathbf{L}} \in \mathcal{L}$, and let $\theta(\tilde{Z}, \overline{\mathbf{L}})$ be the equilibrium technology at factor supplies $(\tilde{Z}, \overline{\mathbf{L}})$ and assume that $\theta(\tilde{Z}, \overline{\mathbf{L}})$ is in the interior of $\Theta$ and that $\partial \theta_{j}(\tilde{Z}, \overline{\mathbf{L}}) / \partial Z$ exists at $(\tilde{Z}, \overline{\mathbf{L}})$ for all $j=1, \ldots, K$ for all $\tilde{Z} \in\left[\bar{Z}, \bar{Z}^{\prime}\right]$. Then there is strong absolute bias at $\left(\left\{\bar{Z}, \bar{Z}^{\prime}\right\}, \overline{\mathbf{L}}\right)$ if $F(Z, \mathbf{L}, \theta)$ 's Hessian, $\nabla^{2} F_{(Z, \theta)(Z, \theta)}$, fails to be negative semi-definite at $(\tilde{Z}, \overline{\mathbf{L}}, \theta(\tilde{Z}, \overline{\mathbf{L}}))$ for all $\tilde{Z} \in\left[\bar{Z}, \bar{Z}^{\prime}\right]$.

Proof. The proof follows from the Fundamental Theorem of Calculus and the proof of Theorem 8. Take $\bar{Z}$ and $\bar{Z}^{\prime}>\bar{Z}$ in $\mathcal{Z}$ and fix $\overline{\mathbf{L}} \in \mathcal{L}$. Then

$$
w_{Z}\left(\bar{Z}^{\prime}, \overline{\mathbf{L}}, \theta\left(\bar{Z}^{\prime}, \overline{\mathbf{L}}\right)\right)-w_{Z}(\bar{Z}, \overline{\mathbf{L}}, \theta(\bar{Z}, \overline{\mathbf{L}}))=\int_{\bar{Z}}^{\bar{Z}^{\prime}} \frac{d w_{Z}(Z, \overline{\mathbf{L}}, \theta(Z, \overline{\mathbf{L}}))}{d Z} d Z .
$$

The hypotheses of the theorem, combined with the proof of Theorem 8 , imply that $d w_{Z}(Z, \overline{\mathbf{L}}, \theta(Z, \overline{\mathbf{L}})) / d Z>0$ for all $Z \in\left[\bar{Z}, \bar{Z}^{\prime}\right]$, so (45) is positive, establishing the result.

The conditions of Theorem 9 are more demanding than Theorem 8, since they require that the Hessian of $F$ with respect to $(Z, \theta)$ should fail to be negative semi-definite at all points $\tilde{Z} \in\left[\bar{Z}, \bar{Z}^{\prime}\right]$. Moreover, this theorem is weaker than Theorem 8 , since it states that failure of negative semi-definiteness of the Hessian of $F$ between $\bar{Z}$ and $\bar{Z}^{\prime}$ is sufficient to ensure strong absolute bias between $\bar{Z}$ and $\bar{Z}^{\prime}$, but does not state that it is necessary (and it is straightforward to check that it is not). This motivated my focus on Theorem 8 for most of the discussion.

\footnotetext{
${ }^{33}$ At this point, we can also return to a further discussion of why the local weak bias result did not translate into a global weak bias result (without imposing further conditions), whereas the strong bias result does (recall the discussion in Example 3). In particular, one might have conjectured that an argument using the Fundamental Theorem of Calculus similar to that in the proof of Theorem 9, in particular, equation (45), may work for weak bias as well. To illustrate why this is not the case, let us suppose that $\Theta \subset \mathbb{R}$. Then:

$$
\frac{d w_{Z}(\bar{Z}, \overline{\mathbf{L}}, \theta(\bar{Z}, \overline{\mathbf{L}}))}{d Z}=\frac{\partial w_{Z}(\bar{Z}, \overline{\mathbf{L}}, \theta(\bar{Z}, \overline{\mathbf{L}}))}{\partial Z}+\frac{\partial w_{Z}(\bar{Z}, \overline{\mathbf{L}}, \theta(\bar{Z}, \overline{\mathbf{L}}))}{\partial \theta} \frac{\partial \theta}{\partial Z}
$$

Equation (45) and Theorem 9 apply to this entire term, while weak bias concerns the second part of this term. It is not possible to apply the Fundamental Theorem of Calculus just to this term, and intuitively, this is the notion discussed in Example 3, whereby an induced change in $\theta$ at some $\bar{Z}$ that is biased towards $Z$ may be biased against $Z$ at some different factor supply, $\bar{Z}^{\prime}$.
} 


\section{Conclusion}

An investigation of the determinants of equilibrium (endogenous) bias is important both for a better understanding of nature of technology adoption and technological change, and to study the distributional implications of new technologies.

In this paper, I analyzed the implications of changes in factor supplies on relative and absolute bias of technology. First, I generalized a number of existing results in the literature about relative bias in two-factor economies. These results are about how the relative marginal product of a factor responds to technological progress or technology adoption induced by changes in factor supplies. In particular, I established that when the economy has access to two technologies, one augmenting factor $Z$ and the other $L$, equilibrium technology will always be relatively biased towards the factor that has become more abundant. Moreover, this induced-bias can be strong enough to increase the relative price of the factor that becomes more abundant. These results are interesting both because they apply to many macro models of economic growth, and also because they are essentially the opposite of the presumption in the literature following Hicks' (1932) seminal work. Nevertheless, the analysis also showed that these results about relative bias do not generalize once we depart from a world with only factor-augmenting technologies. The reason is that induced changes in technology may increase the marginal product of the other factor more than the marginal product of the factor becoming more abundant. This suggests that more general theorems may apply to absolute rather than relative bias.

The second part of the paper shows that this is indeed the case and provides general theorems about absolute bias, i.e., how the marginal product of a factor (rather than its relative marginal product) changes in response to technology. I proved that under mild assumptions, changes in technology induced by small changes in factor supplies are always (absolutely) biased towards the factor that has become more abundant. I also showed that under supermodularitytype assumptions, the same result can be generalized to any magnitude of change in factor supplies, and can be applied to simultaneous changes in the supplies of a set of factors.

Finally, the last section contains the most important results of the paper. It illustrated the possibility of strong (absolute) equilibrium bias. In particular, with strong equilibrium bias, an increase in the supply of a factor induces a sufficiently large change in technology so that the marginal product (price) of the factor that has become more abundant increases; in other words, demand curves for factors become upward sloping. The analysis demonstrated that such strong equilibrium bias is impossible without nonconvexities, but is easy to obtain once 
nonconvexities are present. Moreover, Theorem 8 provided precise conditions for such strong bias to exist, related to the failure of joint concavity of the $F$ function in factor demands and technology, which is possible (and in fact quite typical) in equilibrium environments.

To keep the exposition simple, I have made differentiability assumptions throughout the paper, but the global results can be easily generalized by relaxing differentiability since they were derived using tools from the theory of monotone comparative statics. Another possible generalization is to introduce multiple goods rather than a single final good. This complicates the analysis, but the general insights do not appear to depend on the single good assumption. Yet another interesting generalization might be to integrate some of these results into growth models where there can be long-run growth due to technological change (see Acemoglu, 1998, 2002, 2003b or Jones, 2005, for various growth models with relative equilibrium bias). More important directions for future research include an investigation of the bias of technology in alternative settings where the problem of determining equilibrium technology is not equivalent to a maximization problem. The most important example of this is a strategic setting where there is (oligopolistic) competition between various firms that are also choosing their technologies. Finally, the most important area for future research is an empirical investigation of whether the implications of these strong theorems actually hold in the data. 


\section{Appendix A: Technology Choice with Monopoly in Economy C}

In this appendix, I briefly discuss the results in the environment of Economy $\mathrm{C}$ if the research is undertaken by a profit-maximizing monopolist. The main result is that if we allow the monopolist to charge price schedules rather than a linear price, the result is once again an equilibrium that corresponds to the maximization of some function $F(\overline{\mathbf{Z}}, \overline{\mathbf{L}}, \theta)$.

Recall that the production function is given by (6), with the same assumptions on the function $G$. Suppose that without buying the rights to use some technology, each firm would produce zero output. They can buy these rights from the monopolist technology producer, at some price $\chi$ (I will specify what this price is a function of below). The major difference is that the technology monopolist will choose the technology $\theta$ to maximize its profits rather than social surplus or total output. Since without the technology, a firm produces zero output, the technology monopolist can charge each firm up to a price of $\pi\left(\mathbf{Z}^{i}, \mathbf{L}^{i}, \theta^{i}\right)$. Therefore, its profits are

$$
\Pi(\theta)=\int_{i \in \mathcal{F}} t(i) \pi\left(\mathbf{Z}^{i}, \mathbf{L}^{i}, \theta^{i}\right) d i-C(\theta),
$$

where $t(i)$ is an indicator for whether firm $i$ is buying the new technology. Under the same assumption as in Economy $\mathrm{C}$ that the monopolist can only choose one technology from the menu, it will simply maximize $\Pi(\theta)$. The problem here is that as the monopolist provides better technologies to all firms, they compete more fiercely for the factors of production, so factor prices increase, and as a result, the profits that the technology monopolist can extract decline. For example, if $G$ exhibits constant returns to scale in $(Z, L)$, the monopolist can never extract any positive profits by charging any price schedule $\chi(\theta)$ and selling its new technology to all firms. In fact, in this case, it would clearly be beneficial for the monopolist to charge a price that is not only a function of the technology, but also of the employment levels of the firms, so as to manipulate their factor demand. In particular, suppose that the monopolist can charge firm $i$ a price $\chi\left(\mathbf{Z}^{i}, \mathbf{L}^{i}, \theta\right)$, which is the fee to use technology $\theta$ conditional on firm $i$ employing $\left(\mathbf{Z}^{i}, \mathbf{L}^{i}\right)$. Now consider the following price function for the monopolist

$$
\chi\left(\mathbf{Z}^{i}, \mathbf{L}^{i}, \theta\right)=\left\{\begin{array}{cl}
\pi\left(\overline{\mathbf{Z}}, \overline{\mathbf{L}}, \theta^{i}\right)-\xi & \text { if }\left(\mathbf{Z}^{i}, \mathbf{L}^{i}\right)=(\overline{\mathbf{Z}}, \overline{\mathbf{L}}) \\
\infty & \text { if }\left(\mathbf{Z}^{i}, \mathbf{L}^{i}\right) \neq(\overline{\mathbf{Z}}, \overline{\mathbf{L}})
\end{array}\right.
$$

for some $\xi>0$, and the strategy of selling to a total of $1-\varepsilon$ firms, where $\varepsilon>0$. The price schedule makes it profitable for all firms that are offered the technology to take it, since they will make additional profits equal to $\xi$ by doing so. Since $\varepsilon>0$, there will be excess labor 
supply, so all factor prices will be equal to 0. Consequently,

$$
\sup _{\xi, \varepsilon, \theta} \Pi(\theta)=G(\overline{\mathbf{Z}}, \overline{\mathbf{L}}, \theta)-C(\theta) .
$$

This is written as "sup" not as "max", since the supremum is never reached and the monopolist approaches it as $\xi \downarrow 0$ and $\varepsilon \downarrow 0$. The important result for the analysis is that technology choice is again the solution to the maximization of some function $F(\overline{\mathbf{Z}}, \overline{\mathbf{L}}, \theta)$ (though the supremum is never reached). Even though in this case factor prices are equal to zero, all the results in the text apply to the marginal products of the factors (which are never zero). 


\section{Appendix B: Some Technical Definitions and Results}

In this section, I define some of the terms used in the analysis of global equilibrium bias. The reader is referred to the much more detail discussion in Topkis $(1978,1998)$, and also to Milgrom and Roberts (1990) and Milgrom and Shannon (1994). At the end of the section, I also prove a lemma on negative semi-definite matrices, which is used in the proofs of Theorems 6 and 8.

Let $X$ be a partially ordered set, with an order (reflexive, anti-symmetric and transitive binary relation) denoted by $\geq$ (or $>$ ). For example, $X=\mathbb{R}^{2}$ with the order such that $\left(x_{1}^{\prime}, x_{2}^{\prime}\right) \geq$ $(>)\left(x_{1}, x_{2}\right)$ only if $x_{1}^{\prime} \geq(>) x_{1}$ and $x_{2}^{\prime} \geq(>) x_{2}$ is a partially ordered set. In contrast, $X=\mathbb{R}$ with the natural order $\geq(>)$ is an ordered set or a chain. Let $x^{\prime} \vee x$ denote the join, or the least upper bound of two elements of a partially ordered set $X$. For example, when $X=\mathbb{R}^{2}$, $\left(x_{1}^{\prime}, x_{2}^{\prime}\right) \vee\left(x_{1}, x_{2}\right)=\left(\max \left\{x_{1}, x_{1}^{\prime}\right\}, \max \left\{x_{2}, x_{2}^{\prime}\right\}\right)$. Similarly, the meet, or the greatest lower bound of two elements of a partially ordered set is denoted by $x^{\prime} \wedge x$, and for the case where $X=\mathbb{R}^{2},\left(x_{1}^{\prime}, x_{2}^{\prime}\right) \wedge\left(x_{1}, x_{2}\right)=\left(\min \left\{x_{1}, x_{1}^{\prime}\right\}, \min \left\{x_{2}, x_{2}^{\prime}\right\}\right) . X$ or a subset $S$ of $X$ is a lattice if it contains the join and the meet of each pair of its elements. A subset $X^{\prime}$ of $X$ is a sublattice of $X$ (i.e., a lattice according to the same order over $X$ ) if $X^{\prime}$ contains the joint and the meet of each pair of its own elements.

Let $f: X \rightarrow \mathbb{R}$ be a real-valued function and $X$ be a lattice. Then we have a more general definition of supermodularity than the one in the text:

Definition 14 A real-valued function $f(x)$ defined on a (sub)lattice $X$ is supermodular if

$$
f\left(x^{\prime}\right)+f\left(x^{\prime \prime}\right) \leq f\left(x^{\prime} \vee x^{\prime \prime}\right)+f\left(x^{\prime} \wedge x^{\prime \prime}\right)
$$

for all $x^{\prime}, x^{\prime \prime} \in X$. Moreover, $f(x)$ is strictly supermodular if it satisfies (46) with strict inequality for all unordered $x^{\prime}, x^{\prime \prime} \in X$.

When $f(x)$ is twice continuously differentiable over $X$, the definition for supermodularity is equivalent to the one in the text.

Another useful definition is that of increasing differences, which weakens the supermodularity requirements. ${ }^{34}$

Definition 15 Let $X$ and $T$ be partially ordered sets. Then a function $f(x, t)$ defined on a subset $S$ of $X \times T$ has increasing differences in $(x, t)$, if for all $t^{\prime \prime}>t, f\left(x, t^{\prime \prime}\right)-f(x, t)$

\footnotetext{
${ }^{34}$ The notion of "increasing differences" was originally called isotone in Topkis $(1968,1978)$, and is sometimes referred to as non-decreasing differences (e.g., Amir, 1996).
} 
is nondecreasing in $x$. Moreover, $f(x, t)$ has strictly increasing differences in $(x, t)$, if for all $t^{\prime \prime}>t, f\left(x, t^{\prime \prime}\right)-f(x, t)$ is strictly increasing in $x$.

Clearly, (strictly) increasing differences in in $(x, t)$ and in $(t, x)$ are identical.

In the text, I also made use of the concepts of single crossing property and quasi-supermodularity. These are defined as follows:

Definition 16 A real-valued function $f(x)$ defined on a (sub)lattice $X$ is quasi-supermodular if for all $x^{\prime}, x^{\prime \prime} \in X$,

$$
\begin{aligned}
& f\left(x^{\prime}\right) \leq f\left(x^{\prime} \vee x^{\prime \prime}\right) \Longrightarrow f\left(x^{\prime \prime}\right) \leq f\left(x^{\prime} \wedge x^{\prime \prime}\right), \text { and } \\
& f\left(x^{\prime}\right)<f\left(x^{\prime} \vee x^{\prime \prime}\right) \Longrightarrow f\left(x^{\prime \prime}\right)<f\left(x^{\prime} \wedge x^{\prime \prime}\right)
\end{aligned}
$$

Definition 17 Let $f(x, t)$ be a real-valued function defined on $X \times T$ where $X$ and $T$ are partially ordered sets. Then $f(x, t)$ satisfies the single crossing property in $(x, t)$ if $x^{\prime \prime}>x^{\prime}$, $t^{\prime \prime}>t^{\prime}$ and $f\left(x^{\prime \prime}, t^{\prime}\right) \geq f\left(x^{\prime}, t^{\prime}\right)$ implies that $f\left(x^{\prime \prime}, t^{\prime \prime}\right) \geq f\left(x^{\prime}, t^{\prime \prime}\right)$ and $f\left(x^{\prime \prime}, t^{\prime}\right)>f\left(x^{\prime}, t^{\prime}\right)$ implies that $f\left(x^{\prime \prime}, t^{\prime \prime}\right)>f\left(x^{\prime}, t^{\prime \prime}\right)$.

We have the following result linking supermodularity to increasing differences.

Lemma 2 Suppose that $X$ is a lattice. If $f(x)$ is (strictly) supermodular on $X$, then $f(x)$ exhibits (strictly) increasing differences on $X$. Moreover, suppose in addition that $X \subset \mathbb{R}^{K}$. Then if $f(x)$ exhibits (strictly) increasing differences on $X, f(x)$ is (strictly) supermodular on $X$.

Proof. The first part follows from Theorem 2.6.1 of Topkis (1998), while the second part is an implication of Corollary 2.6.1 of Topkis (1998).

Next, it is useful to state some of the relationships between these concepts invoked in Example 3, and some additional results linking increasing differences to the single crossing property:

Lemma 3 Let $f$ be a real valued function. Then:

1. If $X$ is a lattice and $f(x)$ is supermodular on $X$, then $f(x)$ is quasi-supermodular on $X$.

2. If $X_{1}$ and $X_{2}$ are lattices and $X$ is a sublattice of $X_{1} \times X_{2}$ and $f(x)$ is quasi-supermodular on $X$, then $f(x)$ has the single crossing property in $\left(x_{1}, x_{2}\right)$ and $\left(x_{2}, x_{1}\right)$ on $X$. 
3. If $X_{1}$ and $X_{2}$ are chains and $X$ is a sublattice of $X_{1} \times X_{2}$ and $f(x)$ has the single crossing property in $\left(x_{1}, x_{2}\right)$ and $\left(x_{2}, x_{1}\right)$ on $X$, then $f(x)$ is quasi-supermodular on $X$.

4. If $X_{1}$ and $X_{2}$ are partially ordered sets, $X$ is a subset of $X_{1} \times X_{2}$ and $f\left(x_{1}, x_{2}\right)$ exhibits increasing differences in $\left(x_{1}, x_{2}\right)$ on $X$, then $f\left(x_{1}, x_{2}\right)$ has the single crossing property in $\left(x_{1}, x_{2}\right)$ and $\left(x_{2}, x_{1}\right)$ on $X$.

Proof. See Lemma 2.6.5 of Topkis (1998).

The key theorem for the analysis is the Monotonicity Theorem of Topkis. Here I state a version, which combines elements from Topkis' (1998) Theorems 2.7.1, 2.8.1, 2.8.4, 2.8.6 and Corollary 2.7.1 (see also Topkis, 1978, Theorems 6.1, 6.2 and 6.3). Instead of striving for ultimate generality, I state a version that applies in the context of the problem in the text (e.g., instead of upper semi-continuity, which is necessary for the existence of solutions, I impose continuity etc.).

Theorem 10 (Monotonicity Theorem) Suppose that $X$ and $T$ are lattices and $f(x, t)$ is quasi-supermodular in $x$ and exhibits increasing differences in $(x, t)$ on a compact and complete sublattice $S$ of $X \times T$ and continuous in $x$ on $S$, then $A(t) \equiv \arg \max _{x \in S} f(x, t)$ is a nonempty, compact and complete sublattice of $X$ and is increasing in $t$. Moreover, if $f(x, t)$ is quasi-supermodular in $x$ and exhibits strictly increasing differences in $(x, t)$, then for any $t^{\prime}>t$, $x(t) \in A(t)$ and $x\left(t^{\prime}\right) \in A\left(t^{\prime}\right), x\left(t^{\prime}\right) \geq x(t)$.

Proof. See Topkis (1998).

In the text, I make use of the second part of this theorem which requires $f(x, t)$ to exhibit strictly increasing differences in $(x, t)$, which is only a slightly stronger requirement than increasing differences. With increasing differences, all the results in the text continue to apply except that we only know that the set $A(t)$ is increasing (ascending) in $t$, so all the comparisons have to be for the greatest or the least element of the set $A(t)$. Strict increasing differences ensures that any element of $A\left(t^{\prime}\right)$ is greater than any element of $A(t)$ for $t^{\prime}>t$.

Another useful theorem, first derived by Amir (1996) and generalized by Topkis (1998) Theorem 2.8.5, is the following, which I refer to as the "Strong Monotonicity Theorem". Here again I state a slightly simplified version of the theorem:

Theorem 11 (Strong Monotonicity Theorem) Suppose that $X$ is a convex sublattice of $\mathbb{R}^{n}$ and $T$ is a sublattice of $\mathbb{R}^{m}$, and $f(x, t)$ is quasi-supermodular in $x$, twice continuously differentiable in $(x, t)$ on a compact and complete sublattice $S$ of $X \times T$, continuous in $x$ on $S$, 
and $\partial f(x, t) / \partial x_{i}$ is strictly increasing in $t$ for all $i=1, \ldots, n$, then $A(t) \equiv \arg \max _{x \in S} f(x, t)$ is a non-empty, compact and complete sublattice of $X$. Moreover, if $t^{\prime}, t \in T$ with $t^{\prime}>t$, and $x(t) \in A(t)$ and $x\left(t^{\prime}\right) \in A\left(t^{\prime}\right)$ are in the interior of $X$, then $x\left(t^{\prime}\right)>x(t)$.

Proof. See Topkis (1998) Theorem 2.8.5.

The important feature of this strong monotonicity theorem is that under some additional assumptions, it establishes a strict ordering between $x\left(t^{\prime}\right)$ and $x(t)$, while the original monotonicity theorem only has a weak ordering. ${ }^{35}$

Finally, we have the following lemma, which is used in the proofs of Theorems 6 and $8 .{ }^{36}$ Recall that for a matrix (vector) $v, v^{\prime}$ denotes its transpose.

Lemma 4 Consider the $n \times n$ matrix

$$
B=\left(\begin{array}{cc}
C & v \\
v^{\prime} & b
\end{array}\right)
$$

where $C$ is a $(n-1) \times(n-1)$ symmetric negative definite matrix, $b$ is a scalar, and $v$ is a $(n-1) \times 1$ column vector. Then we have that $B$ is negative semi-definite if and only if $b-v^{\prime} C^{-1} v \leq 0$.

Proof. $(\Longleftarrow) B$ is negative semi-definite if and only if

$$
(x ; y)^{\prime} B(x ; y) \leq 0
$$

where $x$ is an arbitrary $(n-1) \times 1$ vector and $y$ is a scalar, $(x ; y)$ is the $n \times 1$ column vector constructed by stacking $x$ and $y$. Using the form of $B$ in (48), we have

$$
(x ; y)^{\prime} B(x ; y)=x^{\prime} C x+2 y x^{\prime} v+b y^{2} .
$$

When $y=0$, the above expression is always nonpositive since $C$ is negative definite, so $B$ is negative semi-definite as claimed.

Next consider the case where $y \neq 0$. In this case, let $z$ be the $(n-1) \times 1$ vector constructed as $z=x / y$, and let us further expand (49):

$$
\begin{aligned}
(x ; y)^{\prime} B(x ; y) & =y^{2}\left(z^{\prime} C z+2 z^{\prime} v+b\right) \\
& =y^{2}\left(z^{\prime} C z+2 z^{\prime} v+v^{\prime} C^{-1} v\right)+y^{2}\left(b-v^{\prime} C^{-1} v\right) .
\end{aligned}
$$

\footnotetext{
${ }^{35}$ The assumption that $\partial f(x, t) / \partial x_{i}$ is strictly increasing in $t$ for all $i=1, \ldots, n$, is slightly weaker than $\partial^{2} f(x, t) / \partial x_{i} \partial t_{j}>0$ for all $i=1, \ldots, n$ and $j=1, \ldots, m$. Also, the condition that $x\left(t^{\prime}\right)$ and $x(t)$ are in the interior of $X$ can be relaxed along the lines of Theorem 2.8.5 of Topkis (1998).

${ }^{36}$ I thank Alp Simsek for help with the proof of this lemma.
} 
Since $C$ is a real symmetric negative definite matrix, $-C$ is a real symmetric and positive definite matrix, so there exists a non-singular matrix $M$ such that $-C=M^{\prime} M$. Moreover, we also have that $-C^{-1}=M^{-1}\left(M^{\prime}\right)^{-1}=M^{-1}\left(M^{-1}\right)^{\prime}\left[\right.$ since $\left.\left(M^{\prime}\right)^{-1}=\left(M^{-1}\right)^{\prime}\right]$. Now, rewriting equation (50) in terms of $M$, we have

$$
\begin{aligned}
(x ; y)^{\prime} B(x ; y) & =-y^{2}\left(z^{\prime}(-C) z-2 z^{\prime} v-v^{\prime} C^{-1} v\right)+y^{2}\left(b-v^{\prime} C^{-1} v\right) \\
& =-y^{2}\left(z^{\prime}\left(M^{\prime} M\right) z-2 z^{\prime} v+v^{\prime} M^{-1}\left(M^{\prime}\right)^{-1} v\right)+y^{2}\left(b-v^{\prime} C^{-1} v\right) .
\end{aligned}
$$

(51) implies that $B$ is negative semi-definite if and only if

$$
\kappa \equiv y^{2}\left(z^{\prime}\left(M^{\prime} M\right) z-2 z^{\prime} v+v^{\prime} M^{-1}\left(M^{\prime}\right)^{-1} v\right)-y^{2}\left(b-v^{\prime} C^{-1} v\right) \geq 0 .
$$

Now, rearranging terms and with straightforward matrix manipulation, we have

$$
\begin{aligned}
\kappa & \equiv y^{2}\left((M z)^{\prime} M z-2 z^{\prime}\left(M^{\prime}\left(M^{\prime}\right)^{-1}\right) v+\left(\left(M^{-1}\right)^{\prime} v\right)^{\prime}\left(M^{-1}\right)^{\prime} v\right)-y^{2}\left(b-v^{\prime} C^{-1} v\right) \\
& \equiv y^{2}\left((M z)^{\prime} M z-2(M z)^{\prime}\left(M^{-1}\right)^{\prime} v+\left(\left(M^{-1}\right)^{\prime} v\right)^{\prime}\left(M^{-1}\right)^{\prime} v\right)-y^{2}\left(b-v^{\prime} C^{-1} v\right) \\
& \equiv y^{2}\left[\left(M z-\left(M^{-1}\right)^{\prime} v\right)^{\prime}\left(M z-\left(M^{-1}\right)^{\prime} v\right)\right]-y^{2}\left(b-v^{\prime} C^{-1} v\right) .
\end{aligned}
$$

Therefore, $B$ is negative semi-definite if and only if

$$
\kappa \equiv y^{2}\left[\left(M z-\left(M^{-1}\right)^{\prime} v\right)^{\prime}\left(M z-\left(M^{-1}\right)^{\prime} v\right)\right]-y^{2}\left(b-v^{\prime} C^{-1} v\right) \geq 0 .
$$

Now suppose

$$
b-v^{\prime} C^{-1} v \leq 0
$$

then, from equation (52), the first term of $\kappa$ takes the form $y^{2} a^{\prime} a$ for $a \equiv\left(M z-\left(M^{-1}\right)^{\prime} v\right)^{\prime}\left(M z-\left(M^{-1}\right)^{\prime} v\right)$ and is always non-negative for any $z$, so $\kappa \geq 0$, establishing that $B$ is negative semi-definite.

$(\Longrightarrow)$ Conversely, suppose that $B$ is negative semi-definite, which implies that $(x ; y)^{\prime} B(x ; y) \leq$ 0 for all $(x ; y)$. To obtain a contradiction, suppose that

$$
b-v^{\prime} C^{-1} v>0
$$

Then, take $y \neq 0$, and in terms of equation (52), set $z=M^{-1}\left(M^{\prime}\right)^{-1} v$, which yields $\kappa=$ $-y^{2}\left(b-v^{\prime} C^{-1} v\right)<0$ in equation (52), contradicting the hypothesis that $B$ is negative semidefinite (or that $(x ; y)^{\prime} B(x ; y) \leq 0$ for all $(x ; y)$ ), thus yielding a contradiction. 


\section{References}

Acemoglu, Daron, "Why Do New Technologies Complement Skills? Directed Technical Change and Wage Inequality" Quarterly Journal of Economics, CXIII (1998), 1055-1090.

Acemoglu, Daron, "Directed Technical Change" Review of Economic Studies, (2002), 69, 781-810.

Acemoglu, Daron, "Patterns of Skill Premia", Review of Economic Studies, (2003a), 70, 199-230.

Acemoglu, Daron, "Labor- And Capital-Augmenting Technical Change" Journal of the European Economic Association, (2003b), 1, 1-40.

Acemoglu, Daron and Amy Finkelstein, "Input and Technology Choices in Regulated Industries: Evidence from the Healthcare Sector" MIT mimeo.

Acemoglu, Daron and Fabrizio Zilibotti, "Productivity Differences" (2001), Quarterly Journal of Economics, 116, pp.563-606.

Aghion, Philippe and Peter Howitt, "A Model of Growth Through Creative Destruction" Econometrica, 110 (1992), 323-351.

Aghion, Philippe and Peter Howitt, Endogenous Growth Theory, Cambridge, MA, MIT Press, 1998.

Ahmad, Syed, "On The Theory of Induced Invention," Economic Journal 86, (1966), 344357.

Amir, Rabah, "Sensitivity Analysis of Multisector Optimum Economic Dynamics" Journal of Mathematical Economics, 25, (1996) 123-141.

Autor, David, Alan Krueger and Lawrence Katz, "Computing Inequality: Have Computers Changed the Labor Market?" Quarterly Journal of Economics, CXIII (1998), 1169-1214.

Benabou, Roland, "Inequality, Technology and the Social Contract" forthcoming in Handbook of Economic Growth, edited by Philippe Aghion and Steven Durlauf, North-Holland, Amsterdam, 2005.

Binswanger, Hans and Vernon Ruttan, Induced Innovation: Technology, Institutions and Development, Baltimore, Johns Hopkins University Press, 1978.

Boldrin, Michele and David K. Levine, "Perfectly Competitive Innovation," Univesity of Minnesota and UCLA mimeo (2001).

Boldrin, Michele and David K. Levine, "Innovation and the Size of the Market," Univesity of Minnesota and UCLA mimeo (2004). 
Caselli, Francesco and Joan C. Coleman "The World Technology Frontier" Harvard University mimeo, 2004.

Clarke, Frank H. Optimization and Nonsmooth Analysis, 1990, Society for Industrial and Applied Mathematics, Philadelphia.

Dasgupta, Partha and Joseph Stiglitz, "Uncertainty, Industrial Structure and the Speed of R\&D” Bell Journal of Economics, 11 (1980), 1-28.

David, Paul, Technical Choice, Innovation and Economic Growth: Essays on American and British Experience in the Nineteenth Century, London, Cambridge University Press, 1975.

Drandakis, E. and Edmund Phelps, "A Model of Induced Invention, Growth and Distribution" Economic Journal, 76 (1965), 823-840.

Duranton, Gilles, "Economics of Productive Systems: Segmentations and Skill Biased Change", European Economic Review 48 (2004), 307-336.

Farrell, Joseph and Gareth Saloner, "Standardization, Compatibility and Innovation" Rand Journal of Economics, 16 (1985), 70-83.

Gancia, Gino (2003) "Globalization, Divergence and Stagnation" University of Pompeu Fabra working paper.

Goldin, Claudia and Lawrence Katz "The Decline of Non-Competing Groups: Changes in the Premium to Education, 1890 to 1940" NBER Working Papers: 5202

Goldin, Claudia and Lawrence F. Katz, "The Origins of Technology-Skill Complementarity," Quarterly Journal of Economics 113 (1998), 693-732.

Grossman, Gene and Elhanan Helpman, Innovation and Growth in the Global Economy, Cambridge, MA, MIT Press, 1991.

Grossman, Gene and Carl Shapiro, "Dynamic R\&D Competition" Economic Journal, 97 (1987), 372-387.

Jones, Charles I "The Shape of Production Functions in the Direction of Technical Change" Quarterly Journal of Economics 120, 517-550.

Habakkuk, H. J., American and British Technology in the Nineteenth Century: Search for Labor Saving Inventions, Cambridge University Press, 1962.

Hicks, John The Theory of Wages, Macmillan, London, 1932.

James, John A., and Jonathan S. Skinner, "The Resolution of the Labor-Scarcity Paradox," Journal of Economic History, 45 (1985) 513 - 540.

Katz, Michael and Carl Shapiro, "Network Externalities, Competition and Compatibility" American Economic Review, 75 (1985), 424-440. 
Kennedy, Charles, "Induced Bias in Innovation and the Theory of Distribution" Economic Journal, 74 (1964), 541-547.

Kiley, Michael, "The Supply of Skilled Labor and Skill-Biased Technological Progress" 1999, Economc Journal.

Lucas, Robert, "On the mechanics of economic development." Journal of Monetary Economics, 22 (1988), 3-42.

Mantoux, Paul, The Industrial Revolution in the Eighteenth Century: an Outline of the Beginnings of the Modern Factory System in England [translated by Marjorie Vernon], London, J. Cape, 1961.

Mas-Colell, Andreu, Michael D. Whinston and Jerry R. Green, Microeconomic Theory, 1995, Oxford University Press, New York, Oxford.

Milgrom, Paul and John Roberts, "Rationalizability, Learning, and Equilibrium in Games with Strategic Complementarities," Econometrica, 58 (1990a), 1255-1277.

Milgrom, Paul and John Roberts, "The Economics of Modern Manufacturing: Technology, Strategy and Organization," American Economic Review, 80 (1990b), 511-528.

Milgrom, Paul and John Roberts, "The LeChatelier Principle," American Economic Review, 85 (1996), 997-999.

Milgrom, Paul and Chris Shannon, "Monotone Conparative Statistics," Econometrica, 62 (1994), 157-180.

Mokyr, Joel, The Levers of Riches: Technological Creativity and Economic Progress Oxford University Press, New York, 1990.

Nordhaus, William; "Some Skeptical Thoughts on the Theory of Induced Innovation" Quarterly Journal of Economics, LXXXVII, (1973), 208-219.

Quah, Danny, "24/7 Competetive Innovation," London School of Economics mimeo (2002).

Ragot, Xavier, "Technical Change and the Dynamics of The Division of Labor", DELTA Working Papers 2003-09, DELTA (2003).

Reinganum, Jennifer "Dynamic Games of Innovation," Journal of Economic Theory, 25 (1981) 21-24.

Reinganum, Jennifer "Innovation and Industry Evolution," Quarterly Journal of Economics, 100 (1985), 81-100.

Roberts, Kevin "Rationality and the LeChatelier Principle" Journal of Economic Theory, 87 (1999), 416-428.

Romer, Paul M., "Increasing Returns and Long-Run Growth" Journal of Political Economy 
94 (1986), 1002-1037.

Romer, Paul M., "Endogenous Technological Change" Journal of Political Economy, IIC (1990), S71-S102.

Rosenberg, Nathan, Inside the Black Box: Technology and Economics, Cambridge University Press, Cambridge, United Kingdom, 1982.

Rudin, Walter, Principles of Mathematical Analysis, McGraw-Hill, New York, 1964.

Salter W. E.G., Productivity and Technical Change, 2nd edition, Cambridge University Press, Cambridge, United Kingdom, 1966.

Samuelson, Paul, The Foundations of Economic Analysis, Harvard University Press, Cambridge, MA, 1947.

Samuelson, Paul, "An Extension of the LeChatelier Principle," Econometrica, 28 (1960), 368-379.

Samuelson, Paul, "A Theory of Induced Innovations Along Kennedy-Weisacker Lines" Review of Economics and Statistics, XLVII (1965), 444-464.

Segerstrom, P. S., T. Anant, and E. Dinopoulos, "A Schumpeterian Model of the Product Life Cycle" American Economic Review 80 (1990), 1077-1092.

Silberberg, Eugene, "A Revision of Comparative Statistics Methodology in Economic, or, How to Do Comparative Statics on the Bank of an Envelope," Journal of Economic Theory, 7, (1974), 159-173.

Simon, Carl and Lawrence Blume, Mathematics for Economists, WW Norton Co., New York, 1994.

Spence, Michael, "Cost Reduction, Competition and Industry Performance" Econometrica, 52 (1984) 101-122.

Stokey, Nancy, "Human Capital, Product Quality and Growth" Quarterly Journal of Economics, vol. 106, (1991), 587-616.

Stokey, Nancy, "R\&D and Economic Growth" Review of Economic Studies, vol. 62, (1995) 469-490.

Thoenig, Mathias and Thierry Verdier "A Theory of Defensive Skill-Biased Innovation and Globalization" American Economic Review (2003), 93,709-28.

Topkis, Donald, "Minimizing a Submodular Function on a Lattice," Operations Research, 26 (1978), 305-321.

Topkis, Donald, "Equilibrium Points in Nonzero-sum n-person Submodular Games," SIAM Journal on Control and Optimization, 17 (1979), 773-787. 
Topkis, Donald, Supermodularity and Complementarity, Tracing University press, Princeton New Jersey, 1998.

Vives, Xavier, "Nash Equilibrium with Strategic Complementarities," Journal of Mathematical Economics, 19(1990), 305-321.

Young, Alwyn "Invention and Bounded Learning by Doing" Journal of Political Economy 101 (1993), 443-472.

$\mathrm{Xu}$ Bin, "Endogenous Technology Bias, International Trade and Relative Wages", University of Florida mimeo, 2001.

Williamson, Jeffrey, Coping With City Growth During the British Industrial Revolution, Cambridge, Cambridge University Press, 1990. 